\title{
Hemodynamic and volume adaptation to pregnancy : studies in the chronically instrumented conscious rat
}

Citation for published version (APA):

Slangen, B. F. M. (1997). Hemodynamic and volume adaptation to pregnancy : studies in the chronically instrumented conscious rat. [Doctoral Thesis, Maastricht University]. Universiteit Maastricht. https://doi.org/10.26481/dis.19971219bs

Document status and date:

Published: 01/01/1997

DOI:

10.26481/dis.19971219bs

Document Version:

Publisher's PDF, also known as Version of record

\section{Please check the document version of this publication:}

- A submitted manuscript is the version of the article upon submission and before peer-review. There can be important differences between the submitted version and the official published version of record.

People interested in the research are advised to contact the author for the final version of the publication, or visit the DOI to the publisher's website.

- The final author version and the galley proof are versions of the publication after peer review.

- The final published version features the final layout of the paper including the volume, issue and page numbers.

Link to publication

\footnotetext{
General rights rights.

- You may freely distribute the URL identifying the publication in the public portal. please follow below link for the End User Agreement:

www.umlib.nl/taverne-license

Take down policy

If you believe that this document breaches copyright please contact us at:

repository@maastrichtuniversity.nl

providing details and we will investigate your claim.
}

Copyright and moral rights for the publications made accessible in the public portal are retained by the authors and/or other copyright owners and it is a condition of accessing publications that users recognise and abide by the legal requirements associated with these

- Users may download and print one copy of any publication from the public portal for the purpose of private study or research.

- You may not further distribute the material or use it for any profit-making activity or commercial gain

If the publication is distributed under the terms of Article $25 \mathrm{fa}$ of the Dutch Copyright Act, indicated by the "Taverne" license above, 


\section{HEMODYNAMIC \\ AND VOLUME ADAPTATION TO PREGNANCY}

STUDIES IN THE CHRONICALLY INSTRUMENTED CONSCIOUS RAT 
ISBN 90-5681-023-5

(C)B.F.M. Slangen, Maastricht, 1997

Vormgeving binnenwerk: Thijs \& van Wijck, Amsterdam

Printed by Unigraphic, Universiteit Maastricht 


\title{
HEMODYNAMIC \\ AND VOLUME ADAPTATION TO PREGNANCY
}

\section{STUDIES IN THE CHRONICALLY INSTRUMENTED CONSCIOUS} RAT

\author{
PROEFSCHRIFT \\ ter verkrijging van de graad van doctor \\ aan de Universiteit Maastricht, \\ op gezag van de Rector Magnificus, Prof. Mr M.J. Cohen, \\ volgens het besluit van het College van Decanen, \\ in het openbaar te verdedigen, \\ op vrijdag 19 december 1997 om 16.00 uur \\ door \\ Brigitte Francisca Maria Slangen \\ geboren te Heerlen op 11 mei 1968
}


Promotor:

Co-promotor:

Beoordelingscommissie:
Prof. Dr J. de Haan

Dr L.L.H. Peeters

Prof. Dr H.A.J. Struyker Boudier

(voorzitter)

Prof. Dr C.E. Blanco

Prof. Dr A.P.G. Hoeks

Prof. Dr P.W. de Leeuw

Prof. Dr C.P. Weiner

(University of Maryland)

Financial support by the Netherlands Heart Foundation for the publication of this thesis is gratefully acknowledged. 
Voor Carlo

Aan mijn ouders 


\section{CONTENTS}

CHAPTER $1 \quad$ Introduction

page

1

CHAPTER 2: Hemodynamic changes in early pregnancy in

23

chronically instrumented, conscious rats

CHAPTER 3: $\quad$ Aortic distensibility and compliance in

conscious pregnant rats

CHAPTER 4: Blood pressure and heart rate variability in

49

early pregnancy in the rat

CHAPTER 5: The mechanism of vollume adaptation in early

63

rat pregnancy

CHAPTER 6: Hemodynamic changes in pseudopregnancy in

chronically instrumented, conscious rats

Chapter 7: The short-term effect of estradiol-17B on

89

skeletal and renal blood flow in awake,

ovariectomized rats

Chapter 8: General discussion

105

References

115

Summary

135

Samenvatting

Publications

143

Curriculum vitae

Nawoord

149 


\section{Chapter I}

\section{Introduction}




\subsection{General introduction}

1.2 Rat pregnancy and pseudopregnancy

1.3 Endocrine changes in pregnancy

1.3.1 Progesterone

1.3.2 Prolactin

1.3.3 Estrogens

1.3.4 Human Chorionic Gonadotropin

1.4 Endocrine changes in the pseudopregnant rat

1.5 Cardiovascular adaptation to pregnancy

1.5.1 Cardiac output, stroke volume, heart rate, arterial pressure and vascular resistance

1.5.2 Functional and structural changes

1.5.3 Summary

1.6 Renal adaptation to pregnancy

1.7 Volume bomeostasis in pregnancy

1.7.1 Water

1.7.2 Sodium

1.7.3 Plasma osmolality

1.7.4 Plasma volume

1.7.5 Extracellular fluid volume and total body water

1.7.6 Arginine vasopressin

1.7.7 Atrial natriuretic factor

1.7.8 Renin-angiotensin-aldosterone system

1.7.9 Summary

1.8 The initiation of the cardiovascular renal and volume adaptation to pregnancy

1.8.1 Estradiol $-17 \beta$

1.8.2 Progesterone

1.8.3 Prolactin

1.8.4 Human Chorionic Gonadotrophin

1.8.5 Prostaglandins

1.8.6 Nitric oxide

1.8.7 Other factors

1.9 Aims of the study 


\subsection{General introduction}

Pregnancy is characterized by important maternal physiologic adaptations. Cardiac output, stroke volume and heart rate increase while mean arterial pressure and vascular resistance decrease. In addition, there are marked changes in renal hemodynamics, renal function and volume homeostasis, as evidenced by increases in renal plasma flow, glomerular filtration rate and plasma volume. The functional meaning and underlying mechanisms of these profound gestational changes are still poorly understood. Studies in human pregnancy indicate that pregnancy-related hemodynamic changes begin very early in pregnancy (Capeless 1989, Clapp 1988, Duvekot 1993, Robson 1989). It has also been suggested that pregnancies complicated by intra-uterine growth retardation or hypertension are characterized by hemodynamic maladaptations very early in pregnancy, long before clinical symptoms develop (Clapp 1988, Davison 1981a, Duvekot 1993, Easterling 1990). Understanding the mechanisms responsible for the very early adaptations can be expected to provide insight into the pathogenesis of these complications in human pregnancy.

Most studies on hemodynamic changes in human pregnancy focused on late pregnancy. Until recently, it was assumed that the most important maternal adaptations take place in this period, when the metabolic impact of pregnancy on the maternal organism is largest. The earlier studies that supported this view were hampered by the lack of accurate noninvasive methods to quantify cardiovascular changes in a longitudinal study design (Hamilton 1949, Lindhard 1915, Walters 1966). In the last decade several authors have studied early pregnancy hemodynamic changes using modern technology (Clapp 1988, Duvekot 1993, Robson 1989). These studies were started in the 5 th week of human pregnancy. However, several adaptations have already been instituted by the 5 th week and thus their exact onset and time sequence remain undetected. This also implies that the exact role of implantation in the initial hemodynamic adaptation is still obscure. Cardiovascular, renal and volume adaptation to pregnancy has also been studied in other species. The results obtained resemble the changes observed in the human. Advantages of studying a species such as the rat are the relatively short duration of pregnancy ( 23 days), the possibility to use invasive methods and the opportunity to measure before and after implantation (day 6). Furthermore, in this species pseudopregnancy can be studied, which offers a unique model to separate the role of the corpus luteum from that of the conceptus. 


\subsection{Rat pregnancy and pseudopregnancy}

The rat has an estrous cycle of 4 to 5 days, which can be divided into four stages: proestrous, estrous, di-estrous 1 , di-estrous 2 . Mating is only allowed by the female in estrous and leads to conception in more than $90 \%$ (Miller 1980). After the age of 9 months fertility rapidly declines. The ability to reproduce stops by 13 months, before the termination of estrous cycles (Miller 1980). Therefore, the age of all rats used in our studies is chosen to be 3-4 months, when they have reached a body weight of 220-250 grams. After mating, the day of conception is defined as the day of presence of a sperm plug on the cage floor (= day 1 of pregnancy). Implantation of the conceptus occurs on day 6 of pregnancy. Chorioallantoic placentation deve lops between days 10 and 13 of rat pregnancy (Buelke-Sam 1982b, Hebel 1986). Rat pregnancy lasts 22-23 days, and the average litter size is 11 pups (Hebel 1986, Miller 1980).

Rats can readily become pseudopregnant after mating with a vasectomized male. Pseudopregnancy in the rat is characterized by cessation of the estrous cycle and persistence of the endocrine function of the corpus luteum (leucocytic vaginal smear, persistent di-estrus). Termination of pseudopregnancy is characterized by a return to proestrus, usually evident between days 12 and 14 after mating (Atherton 1982a, De Greef 1976, De Greef 1977, Garland 1987).

\subsection{Endocrine changes in pregnancy}

\subsubsection{Progesterone}

In pregnant women, plasma progesterone levels have already increased by week 5 of pregnancy, relative to the luteal phase, and continue to increase (Duvekot 1993, Mishell 1973, Wilson 1980). The plasma progesterone level in rat pregnancy begins to increase within 48 hours after mating, reaching a plateau by day 5 (Bridges 1984, Garland 1987, Miller 1980, Ogle 1977, Pepe 1974). After day 9, plasma progesterone resumes its rise to a new, higher plateau by day 15 . After day 19 the level decreases rapidly (Bridges 1984, Gallo 1985, Garland 1987, Ogle 1977, Pepe 1974, Teeuw 1973). In guinea pig pregnancy, plasma progesterone rises gradually from early pregnancy (day 2) onward (Thapar 1988), only decreasing near term (Hart 1985). 


\subsubsection{Prolactin}

In pregnant women prolactin levels rise early in pregnancy (Biswas 1976). In pregnant rats prolactin is released in two daily surges within hours after mating (Smith 1975), a pattern that continues for 9 days (Smith 1976). On days 10 and 11 only one nocturnal surge can be observed (Smith 1976). The first day on which surges are absent is day 12 . Afterwards the pituitary gland can be removed without inducing abortion (Smith 1976).

\subsubsection{Estrogens}

In pregnant women estradiol-17ß gradually increases from the 5 th to 9 th week of pregnancy onward and remains elevated until term (Duvekot 1993, Mishell 1973, Wilson 1980). Initially, all estradiol is produced by the corpus luteum, as the placenta only begins to produce estradiol after week 6 of gestation (Dewroey 1990). In early rat, guinea pig and sheep pregnancy, the estradiol-17ß level remains low (Bridges 1984, Challis 1971, Gallo 1985, Garland 1987, Rosenfeld 1976, Smith 1975). In rat pregnancy, an estradiol-17ß surge seems to occur on the afternoon of day 4 postmating (Shelesnyak 1963, Yoshinaga 1969) with the largest increase in estradiol-17B concentrations noticeable between days 10 and 15 of rat pregnancy (Bridges 1984, Gallo 1985). The estradiol-17B level remains elevated until term (Bridges 1984, Gallo 1985, Garland 1987, Yoshinaga 1969).

In early guinea pig pregnancy, the peripheral plasma levels of estradiol remain high (preovulatory surge) from conception until day 5 of pregnancy, decreasing again to low or undetectable levels at the time of implantation (days 7-8) (Hart 1985, Thapar 1988). After day 18, estradiol levels begin to increase again until term (Hart 1985).

\subsubsection{Human Chorionic Gonadotropin (hCG)}

In the human, the trophoblast cells secrete hCG shortly after implantation. Peripheral levels of hCG increase rapidly after implantation to reach a peak by the 6 th to 8 th week of pregnancy. In the subsequent 8 weeks hCG decreases rapidly to remain low until term. It is not known whether the rodent produces an hCG-like hormone. Treatment with anti-hCG in the rat induces fetal death, which is more pronounced in early than in late pregnancy (Bambra 1978). 


\subsection{Endocrine changes in the pseudopregnant rat}

In pseudopregnancy endocrine changes are sustained for 10-12 days postmating, closely resembling the changes in the first half of rat pregnancy. The progesterone level begins to increase within 48 hours after mating, to reach a peak between days 5 and 9 , and to return to virgin levels between days 10 and 13 (Atherton 1982a, De Greef 1976, De Greef 1977, Garland 1987, Miller 1980, Pepe 1974, Smith 1975). Prolactin is released in two daily surges which begin within hours after mating, and continue for 11 days (Smith 1975, Smith 1976). On the 12th day one nocturnal surge can be observed. Estrogen levels remain remarkably low in pseudopregnancy (Garland 1987, Smith 1975), except for an estrogen surge on day 4 (Shelesnyak 1963).

\subsection{Cardiovascular adaptation to pregnancy}

\subsubsection{Cardiac output, stroke volume, heart rate, arterial pressure ar vascular resistance}

Unfortunately, almost all studies concerning hemodynamic adaptation to human pregnancy began in the course of the first trimester, well after conception. Cardiac output $(\mathrm{CO})$ has already increased by week 5 after the last menstrual period (Duvekot 1993, Robson 1989) and has increased by 20\% above the nonpregnant value by week 8 (Capeless 1989). By 24 weeks of pregnancy, CO reaches a maximum value of 40 to $50 \%$ above nonpregnant values (Capeless 1989, Easterling 1990, Mabie 1994, Robson 1989, Van Oppen 1996). CO also increases in guinea pig and sheep pregnancy (Curran-Everett 1991, Hart 1985, Myers 1985, Rosenfeld 1977). However, to our knowledge, data in early pregnancy in these species are lacking. Serial studies in the pregnant baboon have shown that $\mathrm{CO}$ increases in the first weeks postconception, but the total rise seems less than in human pregnancy (Phippard 1986).

The maximum rise in $\mathrm{CO}$ observed in near-term pregnant rats is comparable to that observed in human pregnancy (Ahokas 1983, Bruce 1976, Gilson 1992). In the rat, $\mathrm{CO}$ has been reported to remain unchanged in early (Buelke-Sam 1982a, Buelke-Sam 1982b) and even in mid-pregnancy (Ahokas 1983, Dowell 1993). These data may be unreliable because measurements were performed in anes- 
thetized rats, using a single injection of microspheres. Gilson and co-workers measured $\mathrm{CO}$ in pregnancy using the Fick method in chronically instrumented conscious rats (Gilson 1992). They reported a rise in CO by $25 \%$ in midpregnancy, and by $50 \%$ near term. No increase was observed in early pregnancy. The authors explained this by the relatively late implantation in this species. However, conclusions can only be drawn if pregnant, unanesthetized rats are measured serially, using techniques with a smaller measurement error.

Heart rate ( $H R$ ) begins to increase between weeks 5 and 7 of human pregnancy (Clapp 1988, Duvekot 1993, Robson 1989). The increase in HR continues until a plateau of $15 \%$ above the prepregnant value is reached by 32 weeks (Capeless 1989, Robson 1989, Wilson 1980). In contrast, HR does not change in baboon and guinea pig pregnancy (Hart 1985, Phippard 1986). In the sheep and rat it increases by 15-20\%, in late pregnancy only (Dowell 1993, Melone 1991, Rosenfeld 1977).

In human pregnancy, stroke volume (SV) increases gradually from week 8 onward, to reach a maximum of $30 \%$ by 20 weeks (Capeless 1989, Duvekot 1993, Robson 1989). Also in the baboon, SV increases in early pregnancy, in contrast to observations in the rat, guinea pig and sheep, where SV has not been observed to increase before mid-pregnancy (Gilson 1992, Hart 1985, Phippard 1986, Rosenfeld 1977).

In human pregnancy, systolic blood pressure remains relatively constant (Mabie 1994, Robson 1989). Diastolic blood pressure has already decreased significantly by the 8 th week of pregnancy, and reaches a nadir by 28 weeks. In the subsequent weeks it gradually increases to almost prepregnant values at term (Duvekot 1993, Mabie 1994, Robson 1989). Mean arterial pressure (MAP) has decreased by week 4 of baboon pregnancy (Phippard 1986), but changes little in guinea pig pregnancy (Hart 1985). In rat pregnancy, MAP only decreases in the last week (Dowell 1993, Melone 1991, Teeuw 1973). These changes are accompanied by a fall in total peripheral vascular resistance in all species (Gilson 1992, Mabie 1994, Phippard 1986, Robson 1989).

The rise in CO, SV and HR by 8 weeks' gestation represents more than $50 \%$ of the total increase in these variables in human gestation (Capeless 1989). It follows that most of the systemic hemodynamic adaptation is established in very early human pregnancy. Since almost all studies began after week 5 of pregnancy it is obscure whether the initial rise in CO is accomplished by a rise in HR (Duvekot 1993, Robson 1989), a rise in SV, or a combination (Capeless 1989). Serial stud- 
ies in the pregnant baboon indicate that $\mathrm{CO}$ increases in the first weeks of pregnancy by a rise in $\mathrm{SV}$ and a fall in total peripheral resistance, clearly before any measurable increase in blood volume (Phippard 1986). Also in sheep, rat and guinea pig the initial rise in CO seems to result primarily from a rise in SV (Buelke-Sam 1982a, Hart 1985, Rosenfeld 1977). This rise in CO precedes the rise in uteroplacental blood flow in all species studied. It follows that the initial rise in $\mathrm{CO}$ is almost entirely directed towards nonuterine tissues (Hart 1985, Rosenfeld 1977). In term-pregnant guinea pigs and rats, only $15 \%$ of $\mathrm{CO}$ is directed towards the uteroplacental vascular bed (Ahokas 1983, Bruce 1976, Buelke-Sam 1982b, Curran-Everett 1991, Hart 1985, Myers 1985). From these studies it can be concluded that total vascular resistance in pregnancy decreases primarily as a result of a fall in extra-uterine vascular resistance. Several authors have suggested that early pregnancy is characterized by a generalized fall in vascular tone, resulting in a state of relative vascular underfill (Duvekot 1993, Phippard 1986, Schrier 1987). In such a concept, the initial CO rise would be achieved by a rise in HR, with SV gradually increasing in concert with plasma volume expansion. At present, there is insufficient experimental evidence to support this view for human pregnancy. On the other hand, data obtained in early baboon pregnancy, where right atrial pressure has decreased by week 4 of pregnancy, are consistent with a relative underfill (Phippard 1986).

\subsubsection{Functional and structural changes}

In human pregnancy, left ventricular end-diastolic dimension and left atrial diameter have increased by weeks 12 and 8, respectively (Duvekot 1993, Robson 1989). These increases are suggestive of a rise in venous return and cardiac filling pressure, most likely in conjunction with an increase in blood volume. Besides changes in cardiac dimensions there are also changes in cardiac dynamics during pregnancy. In vitro pressure-volume relations in isolated hearts of pregnant guinea pigs show an increase in volume at any given filling pressure throughout pregnancy, which confirms at least in this species the ventricular enlargement (Hart 1985, Morton 1984). Mean velocity of circumferential fiber shortening, an index of myocardial contractility, has already increased by 8 weeks of human pregnancy, and remains elevated until term (Duvekot 1993, Robson 1989). This variable may, however, not provide a reliable index of inherent myocardial contractility, and its validity is limited because of its dependence on heart rate, preload, and afterload. Nevertheless, improved intrinsic myocardial contractility has 
also been demonstrated in vitro in isolated hearts from near-term pregnant rats (Buttrick 1987). The increase in left ventricular mass, already discernable by the 12th week of pregnancy (Duvekot 1993, Robson 1989), would be consistent with a higher cardiac contractility.

Aortic compliance and aortic diameter have increased at the end of human gestation (Hart 1986) and may already be elevated at the end of the first trimester (Hibbard 1994). Data in the rabbit and guinea pig are conflicting: some report no appreciable change (Cha 1992), whereas others note an increase near term (Davis 1989, Goodlin 1984, Humphreys 1994). Data obtained in early pregnancy are lacking. A larger left ventricular size (Hart 1985, Morton 1984) without increase in filling pressure (Davis 1989) in guinea pig pregnancy can be interpreted as an effect of an increased cardiac compliance. These data suggest that both myocardial and total vascular compliance increase in pregnancy.

\subsubsection{Summary}

Most of the increase in CO, HR and SV occurs in early human pregnancy, before the 8th week. A generalized fall in vascular tone leads to a rise in systemic flow, which is almost entirely directed to non-uteroplacental tissues. The rise in $\mathrm{CO}$ seems to precede the rise in blood volume. The instituted high-flow low-resistance circulation is accompanied by myocardial hypertrophy, increased myocardial contractility and increased vascular filling. The impact of increased cardiac and/or arterial compliance on hemodynamic function in early pregnancy is not clear. The hemodynamic adaptations observed in human pregnancy seem to develop in other mammals as well.

\subsection{Renal adaptation to pregnancy}

In human pregnancy glomerular filtration rate (GFR) rapidly increases between the 4 th and 8 th week, to a level of $45 \%$ above the nonpregnant level (Davison 1981a, Duvekot 1993). The raised GFR is maintained throughout gestation, but decreases slightly in the last weeks of pregnancy (Davison 1981a). In other species, similar changes can be observed. In late-pregnant rabbits, GFR and renal blood flow have increased by $40 \%$ and $30 \%$, respectively (Woods 1987). In the rat, the gestational rise in GFR is already noticeable between days 5 and 7 post- 
mating to reach a maximum of $25 \%$ to $40 \%$ above the nonpregnant level by day 12 (Atherton 1.981, Atherton 1983, Baylis 1980, Conrad 1984). This rise in GFR is paralleled by a similar rise in renal plasma flow (RPF) and a corresponding fall in renal vascular resistance (RVR) (Baylis 1980, Baylis 1982, Conrad 1984). GFR, RPF and RVR return toward nonpregnant levels by days 19-20 (Atherton 1981, Conrad 1984, Reckelhoff 1992). The mechanism of the rise in GFR in pregnancy is at present unknown. The autoregulation of renal blood flow and GFR in response to variations in perfusion pressure is preserved in late-pregnant rabbits and midterm and late-pregnant rats (Reckelhoff 1992, Woods 1987). Also, in 12days pregnant rats autoregulation of GFR in response to increments in microperfusion of proximal tubules has not changed compared to nonpregnant rats (Baylis 1985b). Apparently, the setpoint for renal autoregulation has been reset to a lower level of blood pressure and a higher renal blood flow and GFR (Reckelhoff 1992). In order to explore the intrinsic renal mechanisms involved in the rise in GFR it is necessary to perform direct glomerular and tubular micropuncture of a single nephron in laboratory animals. Since filtration pressure equilibrium is preserved in pregnancy (Baylis 1979/1980, Baylis 1980, Baylis 1982) single nephron GFR is entirely dependent on the following three variables: a) the mean transglomerular hydrostatic pressure difference, which in turn can be calculated from the glomerular capillary hydrostatic pressure and proximal tubular hydrostatic pressure; b) the oncotic pressure of blood reaching the glomerulus; and c) the single glomerular plasma flow rate (Baylis 1978, Brenner 1991). Micropuncture studies have revealed that the mean transglomerular capillary hydrostatic pressure and the oncotic pressure have not changed on days 9 and 12 of rat pregnancy (Baylis 1980, Baylis 1982). It therefore appears that the increased single nephron GFR observed in rat pregnancy is entirely due to the increase in single nephron RPF. Similarly, the increase in whole kidney GFR is due to the increase in whole kidney RPF (Baylis 1980, Baylis 1982, Conrad 1984). The latter, in turn, can be secondary to an increased perfusion pressure, plasma volume expansion and/or renal vasodilation. Arterial pressure does not change or even decreases (Atherton 1981, Dowell 1993, Melone 1991, Teeuw 1973), and glomerular capillary blood pressure does not change in rat pregnancy (Baylis 1980, Baylis 1982). Thus, the increased RPF does not seem to be the result from an increased perfusion pressure in early pregnancy. In addition, plasma volume expansion is only modest ( $4 \%$ by day 6 , Baylis $1979 / 1980 ; 12 \%$ by day 9 , Baylis 1982 ), at a time when GFR has reached its maximum (days 6-12) (37\% by day 9, Baylis 1982). As a mat- 
ter of fact, near term, when GFR is declining (Conrad 1984), plasma volume reaches its maximum (Atherton 1981, Atherton $1982 \mathrm{~b}$ ). From these data it can be concluded that there is a dissociation between the increase in plasma volume and that in GFR in rat pregnancy. Also, the increase in GFR precedes the rise in plasma volume and extracellular volume in the baboon and rat (Atherton 1981, Phippard 1986). Furthermore, when virgin female rats are subjected to acute volume loading equivalent to that accumulated by the 9 -day pregnant rat $(=1 \mathrm{ml})$, insignificant changes occur in GFR and RPF (Reckelhoff 1989). The fact that a small volume load does not increase $C O$ and RPF suggests that the mean circulatory filling pressure is not raised, and/or vascular capacitance has increased, at the time of gestational changes in RPF and GFR (Cha 1992, Davis 1989, Douglas 1967, Goodlin 1984, Humphreys 1994). On the basis of these results it seems highly unlikely that the rise in plasma volume triggers the gestational rise in GFR. The unaltered, or slightly decreased, arterial blood pressure together with the near-constancy of the glomerular capillary blood pressure, and the calculated decrease in single afferent arteriole resistance implies that a uniform vasodilation occurs at both the afferent and efferent arterioles (Baylis 1987b, Baylis 1982). We conclude that the increase in GFR in early pregnancy most likely results from an increase in RPF, due to renal vasodilatation. Meanwhile, the autoregulatory renal systems are reset at a lower arterial pressure level, and a higher GFR and RPF.

\subsection{Volume homeostasis in pregnancy}

\subsubsection{Water}

In human pregnancy, the diuresis is higher than in the nonpregnant state from the 12th week onward (Davison 1981b), but returns to the prepregnant level in the third trimester (Davison 1984, Davison 1988). Results on diuresis in rat pregnancy are conflicting: some authors did not find any consistent change (Atherton 1981, Baylis 1980, Conrad 1984, Jansakul 1989), whereas others report an increase in late pregnancy (Atherton 1982b, Dürr 1981). Water intake increases from day 13 of rat pregnancy onward, resulting in a $50 \%$ increase in water intake/24h on day 20 (Atherton 1982b, Dürr 1981). The mean difference between daily water intake and urine output increases progressively from day 14 
onward, resulting in a $56 \%$ positive fluid balance over the last week of pregnancy as compared to the nonpregnant state (Atherton 1982b). If extra-renal fluid loss is assumed to be small, this implies marked fluid retention in the last week of rat pregnancy.

\subsubsection{Sodium}

Plasma sodium concentration has decreased by the 6 th week of human pregnancy (Davison 1984, Davison 1981b, Duvekot 1993). Also in early rat pregnancy plasma sodium concentrations decrease, from the first week onward (Atherton 1982b, Baylis 1980, Conrad 1984, Dürr 1981, Gangull 1969, Lichton 1961). Throughout rat pregnancy sodium intake is increased in conjunction with an increased food intake (Atherton 1982b, Dürr 1981, Kirksey 1962). As a consequence, urinary sodium output is increased in rat pregnancy, but falls in the last week of pregnancy (Atherton 1982b). These changes in sodium-balance result in significant sodium retention, particularly in late pregnancy (Atherton 1982b, Churchill 1978, Kirksey 1962, Lichton 1961). Nevertheless, maternal plasma sodium levels remain slightly lower throughout pregnancy, most likely secondary to resetting of the osmostat. At least $60 \%$ of the retained sodium is needed to meet the demands of the conceptus (Churchill 1978, Lichton 1961). Handling of sodium loads near term does not differ from that in the nonpregnant state (Katz 1973). The early volume expansion and hemodilution in pregnancy do not require sodium, as evidenced by the normal adaptations in rats maintained on zero sodium for the first 12 days of pregnancy (Baylis 1990). Term-pregnant rats subjected to a very low sodium diet, show a smaller decrease in hematocrit, suggesting that extra sodium is needed to sustain the physiologic hemodilution in late pregnancy (Ganguli 1969, Kirksey 1962). Pregnant rats fed a low-sodium diet over the entire course of pregnancy give birth to smaller litters, consisting of growth-retarded pups. In addition, perinatal mortality is raised (Ganguli 1969, Kirksey 1962).

\subsubsection{Plasma osmolality}

Plasma osmolality decreases in the course of human pregnancy (Davison 1984). The decrement in plasma osmolality is already noticeable by the 6th gestational week and reaches its nadir by the 10th week (Davison 1981b, Davison 1988, Duvekot 1993). In rat pregnancy plasma osmolality decreases from day 10 onward, reaching a nadir by day 14 (Atherton 1982b, Barron 1988, Conrad 1984, Dürr 1981). The decrease in sodium concentration accounts for most of the observed fall in plasma osmolality (Barron 1988, Davison 1981b, Dürr 1981). 


\subsubsection{Plasma volume}

In pregnant women, plasma volume has already increased at least $11 \%$ by week 7 of pregnancy and continues to increase gradually afterwards (Clapp 1988, Whittaker 1993). In the second trimester the increase in plasma volume is paralleled by a rise in red blood cell mass. The larger increase in plasma volume is responsible for the development of the "physiological anemia' of pregnancy, reflected in the lower hematocrit in pregnancy (Barron 1984, Del Valle 1993, Longo 1983, Phippard 1986). Plasma volume has already increased by $4 \%, 12 \%$ and $30-50 \%$ on days 6,9 , and 20 of rat pregnancy, respectively (Atherton $1982 \mathrm{~b}$, Barron 1984, Baylis 1979/1980, Baylis 1982, Jansakul 1989, Nadel 1988). In 14days gravid animals, the increase in total blood volume is solely due to expansion of plasma volume. The marked increase in total blood volume $(+54 \%)$ in nearterm pregnant rats is due to increments in both plasma volume $(+74 \%)$ and erythrocyte mass $(+14 \%$ ) (Barron 1984). Also in baboon pregnancy the initial rise in total blood volume is entirely explained by an increase in plasma volume, followed by an increase in red cell volume in advanced pregnancy (Phippard 1986). The increase in plasma volume in early rat pregnancy is not paralleled by measurable fluid retention (Atherton 1981, Atherton 1982b). The demonstration of plasma volume expansion in early pregnant rats subjected to zero sodium intake supports a minor role for salt retention to enable the plasma volume expansion (Baylis 1990). However, quantifying sodium balance over a prolonged period is associated with a large measurement error. Therefore, it is still unsettled whether PV expansion in early rat pregnancy is established by sodium retention or by a shift of extracellular fluid from the interstitium to the intrawascular compartment (Atherton 1982b).

\subsubsection{Extracellular fluid volume and total body water}

In human pregnancy total body water, measured by bioimpedance spectroscopy or deuterium oxide, and extracellular fluid volume, measured by $\mathrm{NaBr}$, increase between the 8 th and 24 th week by $7 \%$ and $14 \%$, respectively (Van Loan 1995). Extracellular fluid volume, measured as inulin space, increases by $63 \%$ (expressed as a percentage of the non-conceptus body weight it increases by only $5 \%$ ), from day 12 of rat pregnancy onward (Atherton 1981, Churchill 1978). 


\subsubsection{Arginine vasopressin/antidiuretic hormone (AVP/ADH)}

Volume and osmolality are important determinants of AVP secretion. Hypovolemia induces a non-osmotic AVP release, whereas a rise in osmolality induces an osmotic AVP release. AVP acts antidiuretic by a direct effect on the reabsorption of water by the kidney (Guyton 1986). Plasma AVP neither changes in human pregnancy (Davison 1988, Davison 1984), nor in rat pregnancy (Barron 1988, Dürr 1981). Results from dehydration studies and saline loading protocols demonstrate that the threshold for AVP secretion is reset to a lower plasma osmolality level in human (Davison 1984, Davison 1988, Dürr 1981) and rat pregnancy (Barron 1988). The plasma AVP responses to fractional decreases in blood volume are similar in 14-days, 21-days pregnant, and nonpregnant rats (Barron 1984). Interestingly, the initial secretory response occurs when absolute intravascular volume is still considerably greater than that of nonpregnant rats (Barron 1984). Since plasma AVP levels do not change in early pregnancy (Davison 1988), it is unlikely that the fall in plasma osmolality at that time results from a non-osmotic AVP release, the latter being triggered by a generalized vasodilation. It is yet unknown whether AVP exerts its effect, without increases in plasma levels, e.g. by increasing the sensitivity of the kidney to AVP. In conclusion, plasma AVP levels are reset to a lower plasma osmolality level, and the increased circulating volume of pregnancy is sensed as normal.

\subsubsection{Atrial natriuretic factor (ANF)}

In the nonpregnant animal, ANF release is triggered by stretching of atrial stretch receptors. Volume expansion induces a rise in ANF output, and with it a natriuretic response and a fall in arterial blood pressure (Guyton 1986). In pregnant women the plasma ANF level increases gradually, resulting in significantly elevated levels by the 20th week (Castro 1994, Miyamoto 1988, Otsuki 1987, Steegers 1991). Data on plasma ANF levels in early and mid-pregnant rats are conflicting (Castro 1989, Jansakul 1989, Kaufman 1991, Nadel 1988, St-Louis 1988). Decreased or unchanged plasma ANF levels in term pregnant rats, compared to nonpregnant rats, is a consistent finding (Castro 1989, Jansakul 1989, Kaufman 1991, Nadel 1988, St-Louis 1988). It is noteworthy that the latter decline takes place at the time that volume expansion is maximal. Apparently, the volume expansion at that time does not raise right atrial pressure (Nadel 1988). As a matter of fact, right atrial pressure decreases in baboon pregnancy (Phippard 1986), and is unaltered in human and sheep pregnancy (Javeshghani 1995, Visser 1991). 
In addition, the natriuretic response and the activation of central pathways in response to atrial stretch are abolished throughout pregnancy in the rat (Deng 1995, Kaufman 1993, Kaufman 1994). In late-pregnant rats a refractoriness to the natriuretic and vasodepressor actions of exogenously administered ANF has been observed (Masilamani 1994), a phenomenon not seen in early pregnancy. The mechanism by which the kidney becomes refractory to the action of ANF is not clear. It is possible that the affinity to, or the number of, ANF receptors decreases. Also, an increased metabolic clearance rate may lower plasma ANF levels (Mukaddam-Daher 1994). It has been suggested that the generalized activation of antinatriuretic systems in the same tubule segment may inhibit the actions of ANF. Assuming an overall relaxation in the vascular bed in pregnancy, including atrial relaxation, the release of ANF will be achieved by a larger volume than in nonpregnant subjects. These adaptations favor sodium retention in the presence of plasma volume expansion.

\subsubsection{Renin-angiotensin-aldosterone system}

By the 8 th week of human pregnancy plasma renin activity has increased relative to the postpartum level and continues to increase until term (Duvekot 1993, Wilson 1980). Plasma aldosterone levels increase between 8 weeks of pregnancy and term (Wilson 1980). Plasma renin activity and plasma aldosterone concentrations show similar patterns of increase in very early baboon pregnancy (week 4) (Phippard 1986). Also in rat pregnancy plasma renin activity increases, from day 3 onward (Garland 1987) and is elevated at term (Conrad 1989a, Garland 1987, Teeuw 1973). The pattern of change in plasma aldosterone levels follows closely that of plasma renin activity (Dürr 1981, Garland 1987). Some authors failed to detect a rise in plasma renin activity in early and midpregnancy in the rat (Nadel 1988, Paller 1984, Teeuw 1973), partly because of the large variations between animals (Paller 1984). Measurement of plasma renin activity, as the rate of angiotensin I production, does not only reflect the changes in renin secretion, but also the changes in plasma renin substrate (Derkx 1987, Wilson 1980). In human pregnancy plasma renin substrate increases (Wilson 1980), while it decreases in rat pregnancy (Barron 1987, Teeuw 1973). Wilson and co-workers estimated the absolute changes in renin secretion in human pregnancy independent of renin substrate, by normalizing plasma renin activity values, using the Michaelis-Menten equation (Wilson 1980). The calculated renin concentration was $3.4 \mathrm{ng} / \mathrm{ml} /$ hour at 8 weeks' gestation and increased to a peak of 5.8 
$\mathrm{ng} / \mathrm{ml} /$ hour at 32 weeks, a rise substantially less than that reported in plasma renin activity (Wilson 1980). They concluded that $50 \%$ of the observed increase in plasma renin activity in human pregnancy is due to the increase in plasma renin concentration, and the rest of the increase is due to a rise in plasma renin substrate (Wilson 1980). This is in agreement with the findings of Duvekot and co-workers, who measured plasma renin concentration by direct radioimmunoassay (Duvekot 1993). In summary, renin secretion increases from early pregnancy onward in human, rat and baboon. The fourfold increases in plasma renin activity overestimates the increase in renin secretion.

\subsubsection{Summary}

The increased plasma volume and blood volume during rat pregnancy are neither associated with suppression of plasma renin activity nor with an increase in plasma ANF. Decreased sensitivity to atrial stretch and the refractoriness to the actions of ANF contribute to the ongoing volume expansion. Apparently, in pregnancy, all volume homeostatic mechanisms are reset to maintain a higher vascular volume.

\subsection{The initiation of the cardiovascular, renal and volume adaptation to pregnancy}

Cardiac output, measured by a single injection of microspheres, and mean arterial pressure do not change in pseudopregnancy (Atherton 1982a, Baylis 1982, Pang 1979). To our knowledge, no studies have been performed in pseudopregnancy measuring cardiac output with more accurate methods.

In the luteal phase of the menstrual cycle GFR increases by 10-20\% (Van Beek 1996, Davison 1981a). Pseudopregnancy can be considered as a prolonged luteal phase. GFR increases between days 7 and 12 of pseudopregnancy and gradually returns to values comparable to virgin rats in the subsequent 3-4 days (Atherton $1982 \mathrm{a}$, Baylis 1982). The magnitude of the increase $(30 \%)$ is almost identical to the rise observed in pregnant rats (Atherton 1982a, Baylis 1982). The pattern of change in GFR closely follows the maternal hormonal changes in pregnancy and pseudopregnancy. Also, the determinants of single nephron GFR, measured by micropuncture, are virtually identical in pregnant and pseudopregnant rats 
(Baylis 1982).

Hematocrit decreases and plasma volume and extracellular volume increase in pseudopregnancy (Atherton 1982a, Baylis 1982). Some investigators report decreases in plasma osmolality in the luteal phase of the menstrual cycle (Vokes 1988), whereas others were unable to demonstrate significant changes (Davison 1981b). The secretory threshold to AVP seems to remain unaltered in pseudopregnancy, although a small fall can not be excluded (Barron 1988). The threshold for AVP secretion is reset to a lower plasma osmolality level in the luteal phase of the menstrual cycle (Vokes 1988). The renal response to atrial stretch, mediated by ANF, is abolished in pseudopregnant rats, as well as in pregnant rats (Kaufman 1993).

These observations strongly suggest that the initiation of the gestational rise in GFR and volume is due to factors of maternal origin rather than of trophoblastic origin. Nevertheless, this does not exclude a major role for some placental factor in preserving the elevated GFR in the second half of rat pregnancy (Alexander 1980, Matthews 1960). Data on hemodynamic changes in early pregnancy and pseudopregnancy are lacking, and thus the role of maternal factors in initiating cardiovascular adaptations remains to be determined.

\subsubsection{Estradiol-17ß}

Estradiol-17ß (E2) is known to have vasodilating properties. The pattern of response of the systemic vasculature in pregnancy resembles the one that can be triggered by this steroid (Davis 1989, Farhat 1996, Hart 1985, LaSala 1989, Magness 1993, Rosenfeld 1976, Slater 1986, Weiner 1994a, 1994b). E2 levels remain remarkably low in early pregnancy (chapter 1.6 ) and the affinity to E2 does not increase with advancing pregnancy (Rosenfeld 1976). Injection of E2 in sheep leads to a rise in SV and $\mathrm{CO}$, without a change in mean arterial pressure (Magness 1989, Rosenfeld 1976, Ueland 1966). It has been shown that E2 induces an increase in the activity of nitric oxide synthases (Goetz 1994, Learmont 1996, Weiner 1994b). It is not clear whether E2 exerts this effect directly by stimulating NO synthase gene expression, or indirectly by increasing flow and with it NO synthase induction via increased shear stress.

Chronic administration of $\mathbb{E} 2$ to gonadal-intact and ovariectomized rats gives rise to slight decreases in plasma sodium and plasma osmolality, and an $8-10 \%$ increase in blood volume (Barron 1986). The effect of E2 on the plasma levels of AVP, the osmotic control of AVP release and the antidiuretic action of AVP are 
conflicting (Barron 1986, Peysner 1990, Wang 1995).

The contribution of E2 to the gestation-induced renal vasodilation is still unknown. In the sheep, E2 does not induce renal vasodilation (Rosenfeld 1976). However, renal blood flow may not be increased in pregnancy in this species (Rosenfeld 1977).

\subsubsection{Progesterone}

It is possible to induce pseudopregnancy in the rat by daily injections of progesterone. Acute and chronic progesterone administration in the rat, resulting in pseudopregnancy, does not lead to changes in GFR (Elkarib 1983). Progesterone administration in the guinea pig, with plasma concentration increased to a level comparable to that in pregnancy, does neither lead to changes in $\mathrm{CO}$ nor to an increase in blood volume (Hart 1985). Left ventricular weight increases in response to prolonged progesterone administration in the guinea pig, suggesting a direct effect of progesterone on the heart (Hart 1985). However, even in combination with E2 CO does not change (Hart 1985, Nakamura 1988). The increase in plasma progesterone levels may contribute to the rise in plasma renin activity through its natriuretic action, which appears to be due to competitive inhibition of aldosterone in the distal tubule (Wilson 1980).

\subsubsection{Prolactin}

The results on the effects of chronic prolactin administration are conflicting. Baylis and co-workers were unable to demonstrate a change in GFR and plasma volume in response to prolactin administration for 7 days, producing a state of pseudopregnancy (Baylis 1985a). Conversely, in other studies, GFR had increased by $\sim 20 \%$ (Elkarib 1983, Walker 1985). It is not clear why these results are so different since equal doses were injected over 7-8 days, and GFR was determined in both studies by micropuncture techniques in anaesthetized rats.

Prolactin administration for a period of 3 weeks induces an increase in blood volume, a decrease in blood pressure and a decrease in angiotensin II responsiveness (Bryant 1973).

Neither pseudopregnancy nor prolonged hormonal treatment with estrone, progesterone, progesterone-estradiol or prolactin has a measurable effect on the threshold for AVP secretion (Barron 1986, Barron 1988, Dürr 1981). Combined treatment with prolactin, estradiol and progesterone mimicking pregnancy lowers the threshold slightly, but much less than in pregnancy (Barron 1988). 


\subsubsection{Human Chorionic Gonadotrophin (hCG)}

$\mathrm{hCG}$ is a potent vasodilator in isolated uterine radial arteries and mesenteric arteries in early and late pregnant rats (Hermsteiner 1997). It has been suggested that hCG not only regulates ovarian and uterine blood flow, but also triggers the adaptive cardiovascular processes seen in early pregnancy (Hermsteiner 1997). hCG administration in nonpregnant women induces a fall in plasma osmolality and decreases the threshold for AVP secretion, to a simillar extent as observed in pregnancy (Davison 1988).

\subsubsection{Prostaglandins}

In both pregnancy and pseudopregnancy, urinary excretion of Prostaglandin E2 increases from day 4 onward (Paller 1984, Paller 1989). However, in chronically instrumented conscious rats, inhibition of prostaglandin synthesis has no effect on renal hemodynamics and mean arterial pressure (Baylis 1987a, Conrad 1986). Results on the refractoriness to vasoconstrictors in pregnancy and its relation to prostaglandin synthesis are conflicting, most likely due to differences in study design (Conrad 1986, Paller 1989). There is no experimental evidence that prostaglandins can be kept responsible for the vasorelaxation observed in pregnancy (Conrad 1986, Kim 1994).

\subsubsection{Nitric oxide (NO)}

The urinary excretion and the plasma levels of nitrate, the stable NO metabolite, and guanosine $3^{\prime}, 5^{\prime}$-cyclic monophosphate (cGMP), the intracellular messenger of $\mathrm{NO}$, increase in the first week of pseudopregnancy and pregnancy in the rat (Conrad 1989b, Conrad 1993). Nitric oxide is generated from the amino acid Larginine by the enzyme NO synthase (NOS) (Moncada 1993). The endothelial isoform of the latter enzyme (eNOS) is located within the endothelium and is involved in the regulation of blood flow (Moncada 1993). In 20 days' pregnant rats eNOS is upregulated in the aorta and mesenteric artery (Xu 1996). Acute and chronic blockade of NO formation by L-NAME during rat pregnancy induces a fall in GFR, RPF and in plasma volume (Baylis 1992, Danielson 1995, Kim 1994, Molnár 1992, Molnár 1994, Pascoal 1995, Umans 1990). Acute NO blockade reverses the pregnancy-induced refractoriness to vasoactive agents (Molnár 1992). Finally, chronic inhibition of NOS in gravid rats leads to the development of hypertension, proteinuria, thrombocytopenia, reduced plasma volume and fetal growth restriction, abnormalities typical for preeclampsia (Baylis 1992, 
Molnár 1994, Yallampalli 1993). Results are conflicting as to whether pregnant rats are more responsive to NO inhibition than nonpregnant rats (Danielson 1995, Molnár 1992, Molnár 1994, Umans 1990).

In contrast to the findings in animal studies, the augmented endothellium-dependent relaxation in omental vessels in human pregnancy is insensitive to inhibitors of prostaglandin, nitric oxide and potassium channels (hyperpolarizing factor) (Pascoal 1996). The role of the other two isoforms of NOS in pregnancy, the neuronal isoform (in the nervous system and other organs such as the kidney), and the inducible isoform (induced by cytokines) is not clear.

In conclusion, NO is definitely involved, most likely as a second messenger, in inducing and maintaining the vasodilated state of pregnancy. The relative contribution of NO probably differs between species.

\subsubsection{Other factors}

Relaxin is a peptide hormone produced by the corpus luteum of pregnancy. In human pregnancy, its secretion pattern parallels that of hCG, with a peak in the first trimester (Quagliarello 1979). It is present in large amount in the second half of rat pregnancy (Sherwood 1980). In nonpregnant spontaneously hypertensive rats, systolic blood pressure is markedly decreased $24 \mathrm{hr}$ after the beginning of intravenous chronic infusion of relaxin (St-Louis 1985, Massicotte 1989). Also, relaxin administration for $42 \mathrm{hr}$ blunts the response to vasoconstrictor agents in the mesenteric vasculature. These responses resemble the changes observed in 20days pregnant rats (Massicotte 1989). However, relaxin does not seem to play a role in the vasodilated state of pregnancy, at least in the rat, since bilateral ovariectomy on day 13 of rat pregnancy does not prevent the late-gestationall fall in blood pressure (Ahokas 1989).

Shortly after ovulation, decidual changes occur in the endometrium, induced by hormones such as progesterone and estrogen. Cytokines probably play an important role in regulating placental development (Starkey 1993). Whether these immunological processes are only important at the feto-placental interface, or also play a role in the systemic adaptations is at present unknown. 


\subsection{Aims of the study}

The objective of this thesis was in the first place to describe the hemodynamic and volume changes in early rat pregnancy and in the second place to explore possible triggers for the initial changes.

The first part consists of four observational studies, to assess the time course and magnitude of hemodynamic (chapters 2,3 and 4) and volume changes (chapter 5) in early rat pregnancy.

The second part focuses on potential factors that might be responsible for the initial hemodynamic changes as observed in part one of this thesis (chapters 6 and 7). 


\section{Chapter 2}

\section{Hemodynamic changes in early pregnancy in chronically instrumented, conscious rats}

Brigitte F.M. Slangen, Iris C.M. Out, Carla M. Verkeste, Louis L.H. Peeters.

Department of Obstetrics and Gynecology, University of Maastricht, $6200 \mathrm{MD}$ Maastricht The Netherlands.

American Journal of Physiology 270: H1779-H1784, 1996. 


\section{Abstract}

To explore the onset of, and the interrelationship between maternal hemodynamic changes in pregnancy, serial hemodynamic measurements were performed in chronically instrumented, conscious rats by using electromagnetic flow probes around the ascending aorta and arterial catheters. The rats were studied daily from day 4 until day 12, and on day 14, 18 and 20 of pregnancy. Nonpregnant (NP) rats, matched for age and days postsurgery served as controls.

In the pregnant $(\mathrm{P})$ group, the hematocrit started to decrease by postconceptional day 6 (day of implantation) to reach a value of $9 \pm 3 \%$ below the initial level, by day 8 . In these 2 days cardiac output (CO) increased by $9 \pm 4 \%$, as a result of a rise in stroke volume (SV). No changes in mean arterial pressure were observed. In both groups, heart rate (HR) had decreased by day 7 . Aortic flow acceleration and peak aortic flow, indicators of myocardial contractility, increased from day 10 on, only in the P group. In this context it should be emphasized that the results of this study do not allow differentiation between the contractile properties of the cardiac muscle and the contractile changes as a result of the altered preload and afterload. By day 12 and day $18, \mathrm{CO}$ had increased by $20 \pm 5 \%$ and $29 \pm 9 \%$, respectively, above the initial value. $\mathrm{CO}$ had increased by $25 \%$ and $40 \%$, respectively, above the value observed in the NP group, by day 12 and day 18 . The rise in $\mathrm{CO}$ after day 14 was accomplished by a concomitant increase in $\mathrm{HR}$ and $\mathrm{SV}$ and was accompanied by a further increase in myocardial performance.

We conclude that hemodynamic changes can already be identified by day 8 of rat pregnancy, 2 days after implantation. The rise in $\mathrm{CO}$ in early pregnancy results from a selective increase in SV and is accompanied by a rise in myocardial performance. 


\section{Introduction}

In early human pregnancy important maternal physiological adaptations occur. The changes in the cardiovascular and renal systems are already evident by the 8 th week of pregnancy. At that time, cardiac output (CO) has increased by $20 \%$ above the nonpregnant value (Capeless 1989), initially due to a rise in heart rate (HR), and after the 5 th week also due to a rise in stroke volume (SV) (Duvekot 1993, Robson 1989). By week 8, mean arterial pressure (MAP) has decreased (Capeless 1989) and the heart begins to show functional and structural changes (Duvekot 1993, Robson 1989). In this period also plasma volume (PV) begins to increase gradually (Whittaker 1993).

Presently, the mechanism responsible for the changes in maternal hemodynamics in early pregnancy is unknown. Because of the practical and ethical constraints upon serial invasive measurements in human pregnancy, a better understanding of this mechanism can be obtained by studying these changes in laboratory animals. Serial studies in the pregnant baboon have shown that $\mathrm{CO}$ begins to increase in the first weeks of pregnancy by a rise in SV, clearly before any measurable increase in blood volume (Phippard 1986). In this respect the initial hemodynamic changes in baboon pregnancy resemble those observed in early human pregnancy (Duvekot 1993, Robson 1989, Schrier 1987). Hemodynamic measurements in early pregnancy in other species are scarce. In the rat, glomerular filtration rate (GFR) increases early in pregnancy (Atherton 1981, Baylis 1979/1980, Conrad 1984), as does PV (Atherton 1982b, Baylis 1979/1980). Because of the resemblance of these changes to those in early human pregnancy (Davison 1981a, Whittaker 1993), the rat is expected to be a suitable model to study the underlying mechanisms of hemodynamic adaptation. However, experimental data on the systemic hemodynamic changes in early rat pregnancy are limited. $\mathrm{CO}$ was found to remain unchanged in early pregnancy as compared to nonpregnant rats (Buelke-Sam 1982a, Gilson 1992). It is possible that early changes in the cardiovascular system have not been detected because rats were not studied longitudinally, and because of the large measurement error associated with the methods used, when employed in small animals. Studies on the systemic hemodynamic changes in mid-pregnancy have led to conflicting results: either CO had not changed (Ahokas 1983, Dowell 1993) or had increased (Gilson 1992). 
The objective of the present study was to determine the onset of, and the interrelationship between maternal hemodynamic changes in early rat pregnancy. To this end, serial hemodynamic measurements were performed in conscious, chronically instrumented pregnant rats by using electromagnetic flow probes around the ascending aorta and arterial catheters implanted on day 1 of pregnancy.

\section{Methods}

ANIMAL PREPARATION Female Wistar rats (Winkelman, Germany) were used, at the age of 3-4 months. All facilities and procedures were approved by the Ethical Board of the University of Limburg, Maastricht. At least one week of acclimatization to the centralized experimental animal facilities was allowed before surgery. The rats were on a day cycle. Surgery was carried out aseptically under ketamine ( $40 \mathrm{mg} / \mathrm{kg}$ ) and xylazine ( $4 \mathrm{mg} / \mathrm{kg}$ ) anaesthesia, according to the method described by Smith and Hutchins (Smith 1979) and Smits et al (Smits 1982). Briefly, under positive pressure respiration a right thoracotomy was performed in the third intercostal space, and the ascending aorta was carefully dissected free from surrounding tissue. By this procedure baroreceptors were not damaged, as evidenced by normal and stable arterial pressure values during measurements. A flow probe $(2.3 \mathrm{~mm}$, Skalar, Delft, The Netherlands) was placed around the aorta at a distance of 3-4 mm from the heart. A silastic drain was inserted and subatmospheric intrathoracic pressure was restored by application of a negative pressure of $10 \mathrm{~cm} \mathrm{H}_{2} \mathrm{O}$. The thorax was then closed and the probe cable was guided subcutaneously to the neck where the connector was secured to the skin. A polyethylene catheter, filled with heparinized saline $(5 \mathrm{IJ} / \mathrm{ml} 0.9 \% \mathrm{NaCl})$, was advanced into the abdominal aorta via the femoral artery. The catheter was then tunneled subcutaneously, exteriorized between the scapulae and plugged with a stainless steel pin.

EXPERIMENTAL PROTOCOL The design of this study was longitudinal. Female rats were mated with an experienced male and presence of a sperm plug was defined as day 1 of pregnancy. Day 6 of pregnancy is the day of implantation. Surgery, as described above, was performed on day 1 of pregnancy. Each pregnant rat $(\mathrm{P})$ was matched with respect to age and body weight with a nonpregnant control rat 
(NP). Weight loss after surgery was less than $5 \%$ and stabilized within 3 days. Rats were studied daily from day 4 through day 12 and then on day 14,18 and 20 , either of pregnancy or postsurgery. After delivery the litter size and the weight and viability of individual pups were recorded.

HEMODYNAMIC MEASUREMENTS Because of diurnal variation in hemodynamics (Smith 1987) all rats were studied at the same time of the day (between 1000 and $1500 \mathrm{~h}$ ). All experiments were performed after calibration of the blood pressure transducer and flow meters. The zero reference point for pressure measurements (Statham pressure transducer) was at the level of the rat's heart. Blood flow through the ascending aorta was measured using an electromagnetic flow meter (Skalar, Delft, The Netherlands). Late diastolic flow through the aorta was taken to be zero, since the probe was positioned just above the heart.

After connecting the arterial catheter and the flow probe to the measurement devices, data were recorded for 90-120 minutes. At the end of this period, hematocrit (Hct; vol.\%; microcapillary method) was measured in duplicate $(100 \mathrm{ml}$ from the arterial catheter). Mean values were stored on hard disk every $30 \mathrm{sec}$ after analysis by a real time data system. From the flow signal, $\mathrm{CO}$ (minus coronary flow; $\mathrm{ml} / \mathrm{min}$ ), HR (bpm), SV (ml), peak aortic flow (PF; $\mathrm{ml} / \mathrm{min}$ ) and aortic flow acceleration $(\mathrm{dF} / \mathrm{dt} ; \mathrm{ml} / \mathrm{s} 2)$ were derived. Systolic pressure, diastolic pressure and MAP (mmHg) were recorded from the arterial pressure signal. Intermittent sampling in early pregnancy interfered with catheter patency. Consequently, the number of consecutive Hct and arterial pressure measurements beyond day 8 was too small to be subjected to statistical analysis.

STATISTICAL ANALYSIS Two-way analysis of variance by ranks (Friedman Test) was used. Wilcoxon Signed Rank Test was subsequently employed to test for changes relative to day 4 within the groups. Differences between the P and NP group were analyzed by Mann-Whitney-U-Test. To correct for the interindividual variation, data were expressed as \% change relative to day 4 postsurgery. Day 4 was selected as the standard day because no differences were observed between P and NP rats by day 4 (Mann-Whitney-U-Test). Data are expressed as means $\pm \mathrm{SE}$, unless stat* ed otherwise. 


\section{Results}

Mean body weight at surgery was $245 \pm 22 \mathrm{~g}$ and $250 \pm 25 \mathrm{~g}$ (means $\pm \mathrm{SD}$ ) in the $\mathrm{P}$ and NP group, respectively. At term, weight in the $\mathrm{P}$ group had increased by $35 \%$, while that of the NP rats had not changed appreciably. The P animals had a litter size of $11 \pm 2$ and pups weighed approx. $6 \mathrm{~g}$. The drop-out rate in the $\mathrm{P}$ and NP group was comparable. Two $P$ rats were left out of the subsequent analysis because of abnormal pregnancy development (resorption of uterine con-

Figure 1

Percentage changes, relative to day 4, in hematocrit (Hct), cardiac output (CO), stroke wolume (SV) and heart rate $(H R)$ in pregnant $(\nabla)$ and nonpregnant rats $(\nabla)$. Values are means $\pm S E$. $P<0.05$ compared with day 4 by Wilcoxon sigmed rank tests, $\$ \mathrm{p}<0.05$ pregnant rats compared with nonpregnant rats by Mann Whitney U Tests.
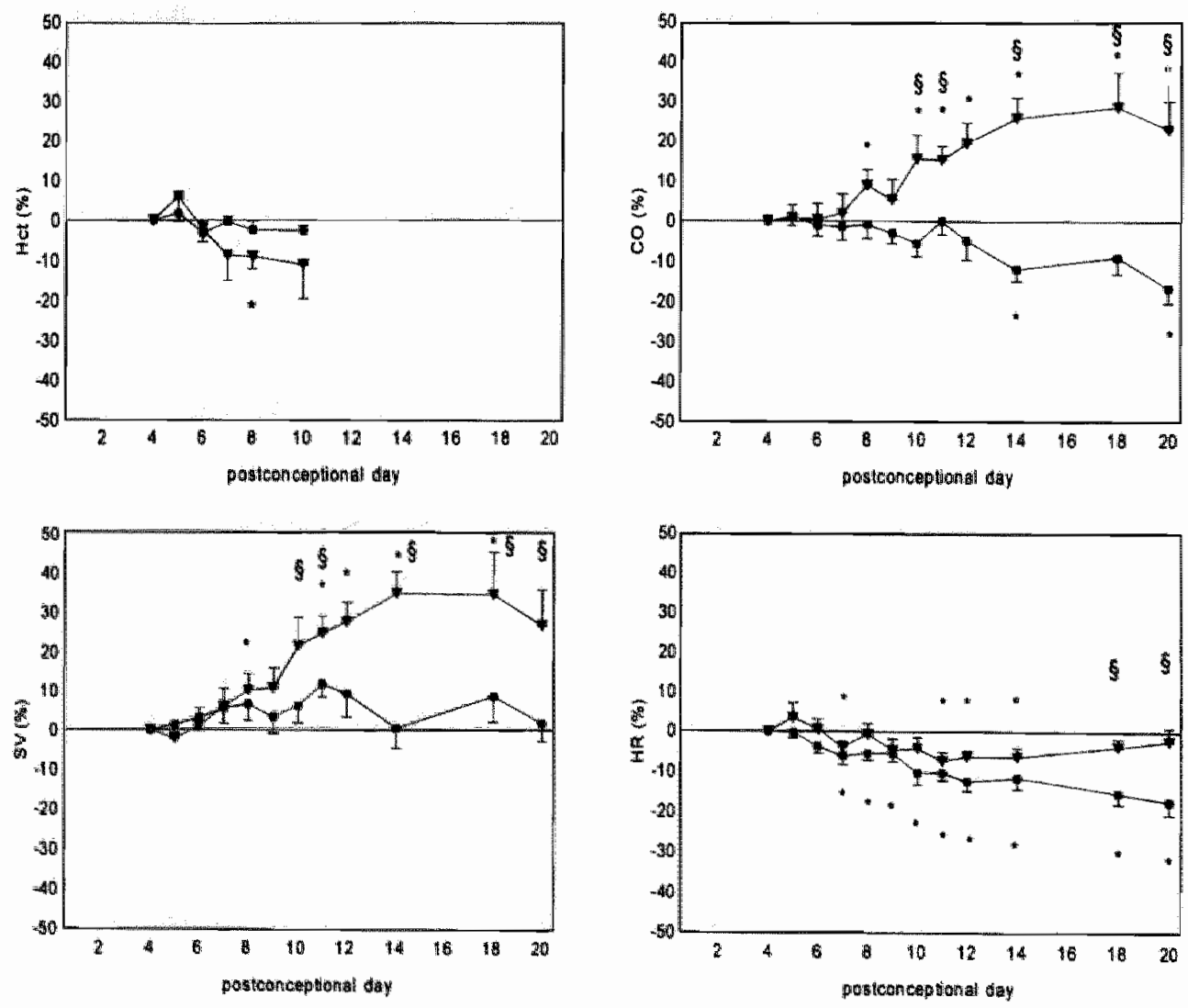
tents, a litter size of 3 pups). One NP rat was excluded because of persistent weight loss. Thus, the number of animals used in the data-analysis for this study is 9 in each group. In $3 \mathrm{P}$ and $2 \mathrm{NP}$ rats, the aorta ruptured after the 10 th day postsurgery. This fatal event is a known acute complication of this type of instrumentation (Smith 1979). Prior to that event, these rats did not differ from their counterparts in their group (normal weight gain, healthy appearance, normal litter size, and similar hemodynamic values). At autopsy there were neither signs of infection nor of fibrosis around the ascending aorta. Therefore, we decided to include the data obtained from these rats in the subsequent anallysis.

Figure 1 depicts the percentage changes with pregnancy (or postsurgery), relative to the 4th day, in Hct, CO, SV and HR. Between days 4 and 6 the changes were small and inconsistent in both groups. In the $\mathrm{P}$ group Hct began to decrease by day 6 , and by day 8 was $9 \pm 3 \%$ below the initial level $(p<0.05)$. This fall in Hct was paralleled by a similar rise in $\mathrm{CO}(9 \pm 4 \% ; \mathrm{p}<0.01)$ and SV $(10 \pm 4 \%$; $\mathrm{p}<0.05$ ) and a small but consistent fall in HR. In the NP group only HR decreased consistently. In neither group did MAP change during this study period (table 1 and 2).

Table 1. Cardiovascular variables in pregnancy

\begin{tabular}{|c|c|c|c|c|c|c|}
\hline & day 4 & day 6 & $d a y s$ & day 10 & day 12 & day 18 \\
\hline $\mathrm{HIR}_{3}$ bears/min & $401 \pm 21$ & $403 \pm 29$ & $397 \pm 21$ & $382 \pm 14$ & $375 \pm 11$ & $387 \pm 19$ \\
\hline $\mathrm{Co}, \mathrm{mi} / \mathrm{min}$ & $71 \pm 7$ & $71 \pm 12$ & $77 \pm 11^{1}$ & $81 \pm 12 \mathrm{~g}$ & $85 \pm 15^{4}$ & $94 \pm 13^{4}$ \\
\hline$S \mathrm{~V}_{3} \mathrm{ml}$ & $176=16$ & $178 \pm 32$ & $194 \pm 27^{\prime \prime}$ & $212 \pm 306^{\circ}$ & $225 \pm 33^{*}$ & $237 \pm 41$ \\
\hline $\mathrm{dF} / \mathrm{dt}, \mathrm{ml} / \mathrm{s} 2$ & $300 \pm 28$ & $296 \pm 41$ & $319 \pm 37$ & $324 \pm 43$ & $336 \pm 48$ & $356 \pm 70 \mathrm{~g}^{\circ}$ \\
\hline pla, milmin & $283 \pm 24$ & $277 \pm 40$ & $298 \pm 34$ & $313 \pm 35 * 5$ & $328 \div 39 \%$ & $344 \div 48 \% 5$ \\
\hline a & $\overrightarrow{9}$ & 9 & 9 & 7 & 9 & 6 \\
\hline $\mathrm{MAP}, \mathrm{mmHg}$ & $102 \div 7$ & $107 \pm 12$ & $107 \pm 12$ & $100 \pm 16$ & - & . \\
\hline Het, vol\% & $37 \pm 3$ & $37 \pm 2$ & $33 \pm 3^{n}$ & $34 \pm 7$ & 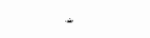 & a \\
\hline$\pi$ & 6 & 8 & $5 / 6$ (hct) & $3 / 2$ (hict) & - & - \\
\hline
\end{tabular}

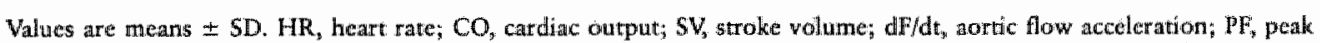
aortic fow; MAP, mean arterial pressure; Hict, hematocric. Eacti wariable was subjected to two-xway analysis of variance by ranks (Friedman Test). $\mathrm{p}<0.001$ for $\mathrm{CO}_{3} \mathrm{SV}$ and $\mathrm{PF} ; \mathrm{p}=0.01$ for $\mathrm{HR} ; \mathrm{p}<0.05$ for $\mathrm{DPF}$ and Hex. $p<0.05$ compared with day 4 by Wilcoxon signed rank tests, $\$ p<0.05$ compared with nonpregnant rats by Manm Whitney U Tetsts. 
Tabile 2. Cardiovascular wariables in exotrols

\begin{tabular}{|c|c|c|c|c|c|c|}
\hline & day 4 & day 6 & day 8 & day 10 & day 12 & doy 18 \\
\hline $\mathrm{HM}_{\text {, beats/min }}$ & $414 \pm 33$ & $398 \pm 23$ & $390 \pm 16^{*}$ & $374 \pm 34^{*}$ & $370 \pm 17^{*}$ & $349 \pm 18$ \\
\hline $\mathrm{CO}, \mathrm{mi} / \mathrm{min}$ & $72=7$ & $71=6$ & $71 \pm 9$ & $67 \pm 75$ & $68 \pm 8$ & $65 \pm 7 s$ \\
\hline$S V_{,} \mathrm{mil}$ & $174 \pm 21$ & $178 \pm 13$ & $184 \pm 17$ & $180 \pm 215$ & $186 \pm 25$ & $186 \pm 225$ \\
\hline $\mathrm{dr} / \mathrm{dt}, \mathrm{mi} / \mathrm{s} 2$ & $311 \pm 28$ & $309 \pm 23$ & $299 \pm 32$ & $289 \pm 33$ & $286 \pm 315$ & $284=275$ \\
\hline $\mathrm{Pf}, \mathrm{mil} / \mathrm{min}$ & $289 \pm 24$ & $283+22$ & $281=28$ & $272 \div 309$ & $273 \pm 3.29$ & $268 \pm 305$ \\
\hline$n$ & 9 & 9 & 9 & 8 & 6 & 7 \\
\hline MAIs: mmHg & $104 \pm 5$ & $106 \pm 8$ & $98 \pm 6$ & $98 \pm 13$ & - & - \\
\hline Het, volos & $37 \pm 1$ & $36 \pm 2$ & $36 \pm 2$ & $36 \pm 2$ & - & - \\
\hline th & 4 & 8/6(het) & $4 / 6$ (her) & $4 / 3$ (hct) & - & - \\
\hline
\end{tabular}

Values are means I SD. HR, heart rate; $\mathrm{CO}$, cardiac output; $\mathrm{SV}$, stroke wolume; $\mathrm{dF} / \mathrm{dt}$, aortic flow acceleration; $\mathrm{PF}$, peak wortic flow: $\mathrm{MAP}_{3}$ mear arterial pressure; $\mathrm{Hct}_{3}$ hematocrit. Each variable was subjected to twomay analysis of wariance by ranks (Friedman Test), $\mathrm{p}=0.001$ for $\mathrm{HR}_{\mathrm{*}} \mathrm{p}<0.05$ for $\mathrm{CO}$. $\mathrm{p}<0.05$ compared with day 4 by Wilcoxon signed rank tests, $\mathrm{p}<0.05$ compared with pregnant rats by Mann Whitncy U Tests.

Between day 8 and day 12, HR continued to decrease to reach a value of $6 \pm 2 \%$ and $13 \pm 2 \%$ below the level of day 4 in the P and NP group, respectively. CO in the $\mathrm{P}$ group had increased by $20 \pm 5 \%(\mathrm{p}<0.01)$ by day 12 , with a rise in SV by $28 \pm 5 \%(\mathrm{p}<0.01)$. In the NP group no change in CO and SV was observed.

By day 18 of pregnancy, $\mathrm{CO}$ and SV had increased by $29 \pm 9 \%$ and $35 \pm 11 \%$ (both $\mathrm{p}<0.05$ ), respectively, relative to day 4 . In the $\mathrm{P}$ group HR was significantly higher than in the NP group on days 18 and 20 .

Figure 2 shows the percentage change with pregnancy or postsurgery, relative to the 4th day, in $\mathrm{PF}$ and $\mathrm{dF} / \mathrm{dt}$, indicators of myocardial performance (Pfeffer 1973, Smith 1979). Again, between days 4 and 6 the changes were small and inconsistent in both groups. $\mathrm{PF}$ and $\mathrm{dF} / \mathrm{dt}$ began to increase gradually by day 7 , in the $\mathrm{P}$ group only. From day 10 until term, $\mathrm{PF}$ and $\mathrm{dF} / \mathrm{dt}$ increased consistently in the $\mathrm{P}$ group, while in the NP group no changes were observed. 
Figure 2

Percentage changes, relative to day 4 , in peak aortic flow (PF) and aortic flow acceleration (dF/dt) in pregnant ( $)$ and nonpregnant rats (-). Values are means \pm SE. * $p<0.05$ compared with day 4 by Wilcoxon signed rank tests, $\$ \mathrm{P}<0.05$ pregnant rats compared with nonpregnant rats by Mamn Whitney U Tests.
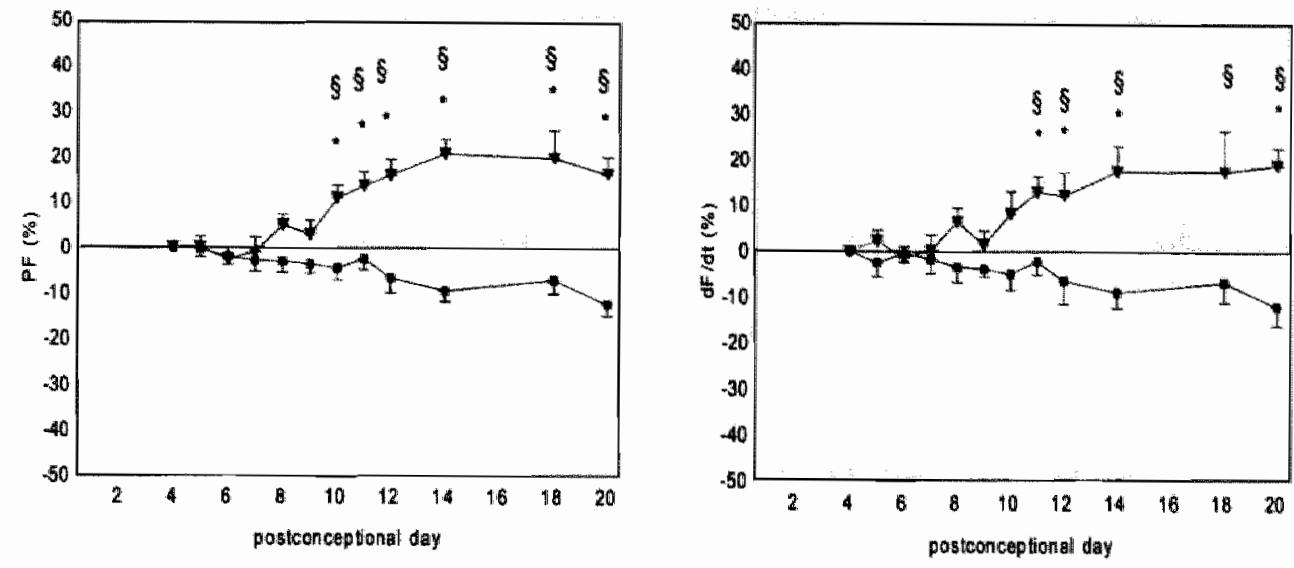

Discussion

The use of electromagnetic flow probes has proven to be a useful technique for longitudinal measurements in chronically instrumented, conscious rats (Smith 1979 , Smits 1982). The values for cardiac index in our nonpregnant rats $(\sim 28$ $\mathrm{ml} / \mathrm{min} / 100 \mathrm{~g}$ body weight, data are not shown) are comparable to those reported previously (Smith 1987). Pregnancy does not seem to be adversely affected by the surgical procedure as evidenced by the low failure rate and high percentage of normal pregnancy outcome. Our results show a gestational rise in cardiac output by $40 \%$ relative to the NP group, an increase comparable to that observed by other investigators in the rat (Ahokas 1983, Bruce 1976, Gilson 1992, Lundgren 1979) and human (Robson 1989).

In the present study, CO had already increased significantly by day 8 by a selective rise in SV. In previous studies in the rat, $\mathrm{CO}$ appeared to be unchanged relative to the NP state in early (Buelke-Sam 1982a) and even in mid-pregnancy (Ahokas 1993, Dowell 1993). These studies were performed under general anaesthesia, a condition known to affect central hemodynamics (Smith 1980). Furthermore, in these studies, only one single value for $\mathrm{CO}$ per animal was obtained using radioactive microspheres, a method associated with a measure- 
ment error of at least 10\% (Buckberg 1971). Recently, Gilson et al measured CO using the Fick method in chronically instrumented, conscious pregnant rats (Gilson 1992). They reported a rise in CO by " $26 \%$ in midpregnancy, an increase comparable to our findings. However, these workers did not find an increase in $\mathrm{CO}$ in early pregnancy. The fact that rats were not studied longitudinally, but were only compared to control groups, together with the larger methodological error in data-acquisition for the Fick methodology, may have left the early rise undetected.

Previous studies of cardiac contractility during pregnancy in the human showed a rise in contractility early in pregnancy (Duvekot 1993, Robson 1989). Slightly improved myocardial performance in near-term pregnant rats has been described by Buttrick in the isolated, perfused working heart preparation (Buttrick 1987), but was not observed in 1-week pregnant rats. The observed rise in $\mathrm{PF}$ and $\mathrm{dF} / \mathrm{dt}$, indicators of myocardial performance (Pfeffer 1986, Smith 1979), in the present study, suggests that the changes can already be observed in midpregnancy, provided data points are collected longitudinally in chronically instrumented pregnant rats. However, from an in vivo study like ours it is not possible to differentiate between the intrinsic contractile properties of the cardiac muscle and the contractile changes as a result of the altered preload and afterload.

Between day 4 and day 6 we neither observed changes within the P group, nor differences between the $P$ and NP rats. Implantation in rat pregnancy occurs on day 6 (Hebel 1986). It is possible that pregnancy-related systemic changes do take place before that particular day, but have remained below the detection level of our methods. This is supported by the observation of an increased GFR, already on day 5 (Conrad 1984).

From day 6 to day $8, \mathrm{CO}$ increased by a selective rise in SV. The increase in SV in early pregnancy may result from a rise in preload, a fall in cardiac afterload, an increase in cardiac contractility or a combination of these phenomena. PV expansion, as suggested by the fall in Hct between day 6 and 8 in our study, and observed on day 7 by others (Atherton 1982, Baylis 1979), can be expected to raise preload. A fall in afterload in early pregnancy (Duvekot 1993) may contribute to the rise in SV. Interestingly, by day 6 of rat pregnancy, plasma renin activity has increased (Conrad 1989a). This supports the view that the initial rise in SV, and with it $\mathrm{CO}$, is inadequate to meet the raised peripheral flow demands. In this context, the lack of a concomitant rise in HR is peculiar, but may be explainable as secondary to a pregnancy-related fall in sympathetic tone or rise in 
vagal activity. In that case, the observed rise in myocardial contractility is probably a consequence of the SV rise. The observed gradual fall in HR in our study in both groups is probably due to habituation of the rat to the instrumentation and the experiments. It is possible that an early rise in HR could not be identified for this reason. In this respect, it is interesting that the fall in HR was less for the $\mathrm{P}$ than for the NP rats. Significant higher values for HR were only observed by day 18 and 20 of pregnancy.

PV expansion is likely to develop gradually due to the raised activity of the volume retaining mechanisms. The latter, again, can be considered a side effect of the primary hyperdynamic circulation of pregnancy (Duvekot 1993, Schrier 1987). PV expansion in early pregnancy can be accomplished by fluid retention, and/or by a shift of extracellular fluid (ECFV) toward the vascular compartment (Atherton 1981, Atherton 1982b). Previous studies have shown that neither fluid intake nor urine output change in the first week of rat pregnancy (Atherton $1982 \mathrm{~b}$ ), implying that fluid retention can not be responsible for the PV expansion by that stage of pregnancy. Therefore, these observations support a rise in PV by a net shift of extracellular fluid into the intravascular fluid. The absence of ECFV expansion in the first week of rat pregnancy (Atherton 1981, Atherton 1982b) further supports this theory. However, the sensitivity of metabolic cage studies might not be high enough to detect small changes in fluid balance in the first week of pregnancy.

In summary, the present study provides experimental evidence for the onset of systemic cardiovascular changes in the rat already by day 8 of pregnancy, two days after implantation. The magnitude of the changes and their onset is comparable to the changes seen in human pregnancy. The rise in $\mathrm{CO}$ in early pregnancy results from a selective increase in SV and is accompanied by a rise in myocardial contractility.

\section{Acknowledgmemts}

We thank Chris Baylis and Kevia Engels, Dept. of Physiology, West Virginia University, for introducing Briginte Slangen to the technical skills reguired in animal research. We thank Jacques Diebers and Elsbeth Raes of the Dept. of Pharmacology, University of Limburg, for teaching the method of chronic implannation of ellectromagnetic flow probes. 


\section{Chapter 3}

\section{Aortic distensibility and compliance in conscious pregnant rats}

Brigitte F.M. Slangen ${ }^{1}$, Dorette S. van Ingen Schenau ${ }^{3}$, Ad W. van Gorp², Jo G.R. De Mey ${ }^{3}$ and Louis L.H. Peeters ${ }^{1}$

Departments of Obstetrics and Gynecology, Physiology2 and

Pharmacology ${ }^{3}$, University of Maastricht, 6200MD Maastricht, The Netherlands.

American Journal of Physiology 272: H1260-H1265, 1997. 


\section{Abstract}

In pregnancy, complex hormonal and hemodynamic changes develop. In this stuidy, we tested the hypothesis that these pregnancy-associated changes are accompanied by an alteration of the compliance of central conduit vessels. To this end, we determined in awake, restrained pregnant rats, the distensibility (DC) and compliance $(\mathrm{CC})$ of the descending thoracic aorta. End-diastolic aortic diameter (d), and changes in aortic diameter during the cardiac cycle ( $\Delta \mathrm{d})$ were measured noninvasively by ultrasound, simultaneously with diastolic aortic blood pressure (Pdia) and pulse pressure $(\Delta \mathrm{P})$ through a chronically implanted catheter. From these parameters, DC and $C C$ (the relative and the absolute increase in lumen cross-sectional area for a given increase in pressure, respectively) were calculated. Nine rats were studied before pregnancy, and on days 4, 8, 10 and 18 of pregnancy, along with 8 nonpregnant rats, matched for age and days postsurgery. On day 20, the animals were sacrificed and the thoracic aorta was isolated for morphometric analysis. In the pregnant group, Pdia decreased from $109 \pm 5$ mmHg on day 4 to $98 \pm 9 \mathrm{mmHg}$ on day 18 (means \pm SD), while end-diastolic aortic diameter did not change. $\Delta \mathrm{d}$ increased from $242 \pm 20 \mathrm{~mm}$ on day 4 to 271 $\pm 29 \mathrm{~mm}$ on day 18 . $\triangle \mathrm{P}$ did not change. Both $\mathrm{DC}$ and $\mathrm{CC}$ increased in early pregnancy to a plateau reached by day 10 . The increase in DC and CC preceded the fall in Pdia. On day 20, neither cross-sectional area nor thickness of the aortic media had changed significantly, when compared with the nonpregnant group. In the nonpregnant group, none of the variables changed consistently during the study period. On the basis of these results we conclude that the compliance of the thoracic aorta increases in early pregnancy. We speculate that the increased aortic compliance and distensibility and the decreased total peripheral resistance develop in early pregnancy in response to the increase in stroke volume, thus preventing a concomitant rise in arterial wall stress. 


\section{Introduction}

Maternal cardiovascular adaptation to pregnancy has been demonstrated to initiate in early pregnancy (Capeless 1989, Davis 1989). In the rat, cardiac output has already increased by day 8, two days after implantation (Slangen 1996). This rise is a result of a selective increase in stroke volume (Slangen 1996). Furthermore, total peripheral resistance decreases (Gilson 1992) and plasma volume increases (Atherton 1981, Baylis 1979/1980). This state of a high flow-low resistance circulation is paralleled by an unchanged or slightly reduced blood pressure (Conrad 1992, Duvekot 1993). It is not clear whether the observed fall in afterload in pregnancy (Duvekot 1993) is solely due to a fall in resistance in the distal portion of the arterial bed. Increased aortic compliance, resulting from aortic smooth muscle relaxation and/or dilation of the vessel in response to the elevated aortic blood flow (Langille 1989, Langille 1990), may contribute as well.

Aortic compliance is increased at the end of human gestation (Hart 1986) and may already be elevated at the end of the first trimester (Hibbard 1994). In latepregnant guinea pigs (Cha 1992, Davis 1989) and rabbits (Humphreys 1994), total vascular compliance is unchanged (Cha 1992) or even increased (Davis 1989, Humphreys 1994). Data in early pregnancy in any species are, however, still lacking.

In this study, we evaluated whether the compliance of large central arteries is modified in early pregnancy. To this end, we measured aortic compliance and distensibility serially in awake, pregnant rats, using a recently developed noninvasive ultrasound technique (Van Gorp 1995, Van Gorp 1996).

\section{Materials and Methods}

ASSESSMENT OF AORTTC DISTENSIBILTTY AND COMPLIANCE. Distensibility and compliance of an artery can be defined as the relative and absolute increases in arterial volume for a given increase in pressure during the systolic phase of the cardiac cycle, respectively. Since it is practically impossible to accurately determine volume and volume changes noninvasively in vivo, distensibility and compliance are generally determined by assessing changes in lumen cross-sectional area ( $\triangle \mathrm{A} / \mathrm{A}$ and $\Delta \mathrm{A}$, respectively) per unit change in pressure. This simplification is allowed 
when one assumes that the increase in arterial wolume during the cardiac cycle is caused by an increase in lumen cross-sectional area rather than by lengthening of the artery (Reneman 1986). $A$ and $\triangle A$ can be derived from the end-diastolic diameter (d) and the changes in diameter of the artery during the cardiac cycle $(\Delta \mathrm{d})$, provided that the vessel lumen keeps a circular cross-section throughout the cardiac cycle. In the remainder of the text, $\Delta \mathrm{A} / \Delta \mathrm{P}$ and $(\Delta \mathrm{A} / \mathrm{A}) / \Delta \mathrm{P}$ will be referred to as the compliance coefficient $(C C)$ and distensibility coefficient (DC), respectively.

The non-invasive ultrasound technique to assess $\mathrm{d}$ and $\Delta \mathrm{d}$ of the thoracic aorta in rats has recently been described in detail (Van Gorp 1996). Briefly, the ultrasound device consists of a conventional B-mode ultrasound system (Pie480, 7.5 MHz linear array, Pie Medical, Maastricht, the Netherlands) combined with a vessel wall tracking system. The acquisition system samples ultrasound and pressure data simultaneously. Since the wall tracking system and the intra-aortic catheter displayed different time responses and were located at different positions along the aorta, we limited the analysis of the relationship between aorta diameter and intra-aortic pressure to the values reached at the end of diastole, and at peak changes in diameter and pressure.

MEASUREMENT OF AORTIC DISTENSIBILTTY AND COMPLIANCE. The rats are placed in a restraining cage (Van Gorp 1996). The ultrasound probe is placed on the thorax parallel to the sternum, after the application of ultrasonic gel on the skin. The thoracic aorta is then visualized in B-mode (B: brightness) approximately $10 \mathrm{~mm}$ above the diaphragm, and the probe is positioned so that the M-line (M: motion) is perpendicular to the vessel wall. Then, the ultrasound system is switched to Mmode, and ulltrasound is emitted and received along the selected line of sight at a programmable emission trigger frequency. The anterior and posterior vessel wall are displayed graphically, allowing manual identification of the transition between wall and blood column by placing two markers, representing the sample windows for data processing. From the initial distance between the sample windows and the observed distension waveform, the end-diastolic diameter and the change in diameter during the cardiac cycle can be derived for each cardiac cycle. Measurements are made six times at short intervals during 1.2 seconds $(\sim 8$ heart beats). The ultrasound probe was removed from the body in between the measurements. 
EXPERIMENTAL PROTOCOL. The measurements were performed in 9 pregnant $(\mathrm{P})$ and 8 nonpregnant (NP) Wistar rats (Iffa Credo, Broekman Institute, Someren, The Netherlands). All experimental procedures were approved by the Institutional Animal Care and Use Committee of the University of Limburg. The animals were maintained on a 12-hour light, 12-hour dark cycle, fed standard rat chow (Hope Farms, Woerden, The Netherlands) ad libitum and had free access to tap water.

The rats were trained for 5 weeks (from 1.0 weeks of age) to familiarize them with the restraining cages in which they were kept during the measurements. The restrainers have an opening on the ventral side for the application of the probe and are equipped with a platform at the rear end to rest their feet on (Van Gorp 1996). The rats were restrained for $10,20,30,40$ and 50 minutes, during the first 5 days, and for 1 hour on mondays, wednesdays and fridays during the remaining 4 weeks of training. In the last 2 weeks the thorax of the rats was shaved and they were also trained to get used to the application the probe together with the gel. In the last week, a pre-surgical measurement was performed. Despite training the rats for 5 weeks, the measurements remained stressful, as judged from measurements of blood pressure, heart rate and plasma catecholamines in the same rats under freely moving conditions and during echomeasurements (Van Gorp, Schiffers, De Mey; unpublished observations).

After 5 weeks of training and after one measurement before surgery, rats were mated with a male. Day one of pregnancy was defined as the day of presence of a sperm plug. On this day, surgery was carried out aseptically under ketamine (40 $\mathrm{mg} / \mathrm{kg}$ ) and xylazine $(4 \mathrm{mg} / \mathrm{kg})$ anesthesia. A poly-ethylene catheter $(0.28 \mathrm{~mm} \mathrm{ID;}$ $0.61 \mathrm{~mm}$ OD; Portex, Medica BV, Den Bosch, The Netherlands), filled with heparinized saline $(5 \mathrm{IU} / \mathrm{ml} 0.9 \% \mathrm{NaCl}$ ), was advanced into the abdominal aorta via the left femoral artery. The catheter tip was positioned below the orifice of the left renal artery. The catheter was then tunneled subcutaneously, exteriorized between the scapulae and plugged with a stainless steel pin.

Measurements were performed on days $4,8,10$, and 18 of pregnancy and/or postsurgery in both $\mathbf{P}$ and NP rats. Rats were placed in the restrainer and the arterial catheter was connected to a blood pressure transducer (CP-01, Century Technology, Inglewood, CA, USA). Measurements were started after the rat had stabilized within the experimental environment. At the end of this period hematocrit $(\mathrm{Hct})$ (vol\%; microcapillary method) was measured in duplicate $(100 \mathrm{ml}$ from the arterial catheter). 
One $\mathrm{P}$ rat and one NP rat could not be measured on day 18. The aorta of the $\mathrm{P}$ rat could not be visualized on day 18 , because of the increase in abdominal size, impeding reliable ultrasound measurement. The dropout of the NP rat was due to catheter failure.

AORTYC WALL STRUCTURE. On day 20 of pregnancy and/or postsurgery, the rats were euthanized by an overdose ether anesthetics. An approx. $10 \mathrm{~mm}$ supradiaphragmatic segment of the thoracic aorta was dissected, fixed in $4 \%$ neutral buffered formaldehyde, and embedded in paraffin, after which $4 \mu \mathrm{m}$ thick sections were obtained. These sections were stained with Lawson's solution (Boom B.V, Meppel, The Netherlands), to highlight elastic laminae and to measure the crosssectional area of the media. Media cross-sectional area was measured using an axiolpan microscope (Zeiss) equipped with a standard CCD camera (Stemmer). Digitized images were analyzed with commercial software (JAVA, Jandel Scientific, Coste Madre, CA, USA). The area enclosed by the external and internal elastic laminae was considered to represent the ex vivo cross-sectional area of the media $\left(\mathrm{CSA}_{\mathrm{e}}\right.$ ). This was converted to in vivo media cross-sectional area (CSA $\mathrm{A}_{\mathrm{i}}$ ) by the formula

$$
\operatorname{CSA}_{\mathrm{i}}=\left(\mathrm{L}_{\mathrm{e}} / \mathrm{L}_{\mathrm{i}}\right) \cdot \mathrm{CSA}_{\mathrm{c}}
$$

assuming no effect of isolation and fixation on aortic wall volume. In this formula $L_{i}$ and $L_{e}$ refer to the length of the thoracic aorta before and after isolation, respectively. To this end, a segment of exactly $10 \mathrm{~mm}\left(\mathrm{~L}_{\mathrm{i}}\right)$ halfway between heart and diaphragm was marked on the aorta and subsequently removed from the rat and cleaned of adhering fat and connective tissue, after which the ex vivo length of the segment $\left(\mathrm{L}_{\mathrm{e}}\right)$ was again measured under a stereomicroscope with a precision of $\sim 5 \mu \mathrm{m}$. $\mathrm{L}_{\mathrm{e}} / \mathrm{L}_{\mathrm{i}}$ averaged $0.76 \pm 0.01$ in $\mathrm{P}$ rats and $0.77 \pm 0.03$ in NP rats (means $\pm S D$ ). Media thickness $(\mathrm{Mt})$ in diastole was calculated from the ultrasound measurement of end-diastolic diameter (d) and from CSAi, using the formula

which can be rewritten as

$$
\operatorname{CSA}_{i}=\pi\left(d / 2+M_{t}\right)^{2}-\pi(d / 2)^{2}
$$

$$
M_{t}=\left[(d / 2)^{2}+\mathrm{CSA}_{i} / \pi\right]^{1 / 2}-(d / 2)
$$


In these calculations it is assumed that the cross-section of the aorta was circular in situ.

We combined data on blood pressure with $\mathrm{d}$ and Mt measurements to compute aortic wall stress (WS) using the Law of Laplace

$$
\mathrm{W} / \mathrm{S}=(\mathrm{P} \cdot \mathrm{d} / 2) / \mathrm{M}_{\mathrm{r}}
$$

where $P$ is pressure, $d$ is aortic diameter and $M_{t}$ is media thickness. WS, P, $d$, and $M_{t}$ were calculated for diastole as well as systole (Nichols 1990 , Zarins 1987).

STATISTICAL ANALYSIS. All ultrasound measurements were performed by one and the same observer. In each rat, six consecutive 1.2 second recordings of pressure, internal lumen diameter, and distension were obtained and derivates calculated for each individual cardiac cycle. Mean values of these six recordings were calculated for each session. The coefficients of variation between the six measurements on one day ranged between $3.8 \%$ and $5.9 \%$ for $d$ and between $4.1 \%$ and $7.1 \%$ for $\Delta \mathrm{d}$. Variabilities did not differ between groups, and did not change after catheter implantation or pregnancy. The coefficients of variation between the four sessions on the four experimental days (days $4,8,10$ and 18 postsurgery) in the $8 \mathrm{NP}$ rats was $4.1 \%$ for $\mathrm{d}, 3.8 \%$ for $\Delta \mathrm{d}, 5.9 \%$ for DC and $5.5 \%$ for CC. End-diastolic diameter and distension before surgery were compared with those on day 4 by Wilcoxon's signed rank test. Each variable was subjected to two-way analysis of variance by ranks (Friedman Test; days 4, 8, 10, and 18 postsurgery). Wilcoxon's signed rank test was subsequently employed to test for changes relative to day 4 within the groups. Differences between the $\mathrm{P}$ and NP group were analyzed by Mann-Whitney U-test. Data are expressed as means $\pm S D$, unless stated otherwise. Differences were considered significant when the P was less than 0.05 .

\section{Results}

Mean body weight at surgery was $222 \pm 6 \mathrm{~g}$ and $226 \pm 10 \mathrm{~g}$ in the $\mathrm{P}$ and NP group, respectively. By day 18 , weight in the $\mathrm{P}$ group had increased to $279 \pm 10$ $\mathrm{g}$, while that in the NP group had not changed significantly. The $\mathrm{P}$ rats had a litter size of $10 \pm 1$ (mean \pm SE) pups.

Table 1 summarizes the observations in the $\mathrm{P}$ and NP groups before surgery and on days $4,8,10$, and 18 of pregnancy and/or postsurgery. Before surgery 
(=before pregnancy), $\mathrm{d}$ and $\Delta \mathrm{d}$ did not differ between the $\mathrm{P}$ and NP group. In the NP group, none of the variables changed consistently throughout the experimental period. In pregnancy, diastolic blood pressure (Pdia) decreased from $109 \pm 5 \mathrm{mmHg}$ (day 4) to $98 \pm 9 \mathrm{mmHg}$ (day 18), while d did not change. $\Delta d$ increased from $242 \pm 20 \mathrm{~mm}$ on day 4 to $271 \pm 29 \mathrm{~mm}$ on day 18 , a $12 \%$ increase (figure 1). $\triangle P$ did not change. Both $D C$ and $C C$ increased in early pregnancy to reach a plateau by day 10 . These alterations in $D C$ and $C C$ preceded most of the change in blood pressure (figure 1 ).

The aortic media cross-sectional area and media thickness at day 20 postsurgery were not significantly different between the $P$ and NP groups (table 2). Despite the fall in diastolic blood pressure towards the end of pregnancy and the unchanged aortic diameter and media thickness, arterial wall stress did not decrease significantly at any time during the cardiac cycle (table 2).

\section{Discussion}

We measured in vivo aortic wall properties in conscious pregnant rats by a new ultrasound technique. This type of ultrasound system can quantify displacements of a few micrometers (Hoeks 1990) with excellent reproducibility as indicated by the small variability between measurements $(<6 \%$ ) (see Methods and Van Gorp 1996). The values for Pdia, $d$ and $\Delta d$ found in our study are slightly smaller than the values found in previous studies in awake male Wistar Kyoto rats (Van Gorp 1996). In these rats (weighing $299 \mathrm{~g}$; aged 12 weeks) Pdia was $-99 \mathrm{mmHg}$, $\mathrm{d}$ was $-1.84 \mathrm{~mm}$, and $\Delta \mathrm{d}$ was $-288 \mathrm{~mm}$, while we found $-112 \mathrm{mmHg}, \sim 1.65 \mathrm{~mm}$ and $-230 \mathrm{~mm}$, respectively. Whether this difference in aortic function can be attributed to sex, strain or weight differences is yet unclear. A disadvantage of this technique is that, despite training the rats for 5 weeks, the measurements seem to remain stressful, as evidenced by the high heart rate of -540 beats/min (data not shown) compared to -390 beats/min in our previous study (Slangen 1996). This has to be taken into account during interpretation of the findings. On the other hand, normal hemodynamic adaptation to pregnancy in the present study is evidenced by the fall in MAP and Hot (table 1), as described previously (Conrad 1992, Slangen 1996). Also, the normal litter size at day 20 confirms the normal development of pregnancy. 
Table 1 Aortic distensibility awd compliance in pregrancy

\begin{tabular}{|c|c|c|c|c|c|c|}
\hline & & Before surgery & $d a y+4$ & day 8 & day 10 & day 18 \\
\hline \multicolumn{7}{|l|}{$\Delta d d_{1}, \mu n n$} \\
\hline & $\mathrm{P}$ & $237 \pm 14$ & $242 \pm 20$ & $254=18^{*} t$ & $264 \pm 19^{2}$ & $271 \pm 29^{*}$ \\
\hline & NP & $227 \pm 13$ & $233 \pm 21$ & $230 \pm 22$ & $232 \pm 22$ & $239 \pm 20$ \\
\hline \multicolumn{7}{|l|}{$\mathrm{d}, \mathrm{mm}$} \\
\hline & $\mathbf{P}$ & $1.63 \pm 0.08$ & $1.65 \div 0.08$ & $1.62 \pm 0.09$ & $1.62 \pm 0.06$ & $1.62 \pm 0.11$ \\
\hline & $\mathrm{NP}$ & $1.64 \pm 0.06$ & $1.65 \pm 0.09$ & $1.67 \pm 0.12$ & $1.63=0.09$ & $1.65+0.08$ \\
\hline \multicolumn{7}{|l|}{ Pdia, mmHg } \\
\hline & $\mathbf{P}$ & . & $109 \pm 5$ & $105 \pm 4^{*}$ & $103 \pm 6^{*}$ & $98 \pm 9^{4}+$ \\
\hline & $\mathrm{NP}$ & - & $114 \pm 8$ & $114 \pm 6$ & $112 \pm 6$ & $112 \pm 4$ \\
\hline \multicolumn{7}{|l|}{$\mathrm{AP}, \mathrm{mmHg}$} \\
\hline & P & . & $56 \pm 4$ & $55 \pm 2$ & $54 \pm 4$ & $55 \pm 4$ \\
\hline & NP & - & $58=2$ & $57 \pm 3$ & $57 \pm 4$ & $60 \pm 3$ \\
\hline \multicolumn{7}{|l|}{$\mathrm{DC}, 10^{.3} 1 / \mathrm{kPa}$} \\
\hline & $\mathrm{P}$ & . & $43 \pm 4$ & $46 \pm 4^{*} t$ & $50 \pm 4 *+$ & $50 \pm 7^{*}+$ \\
\hline & $\mathrm{NP}$ & - & $40 \pm 4$ & $40 \pm 5$ & $41 \pm 5$ & $39 \pm 4$ \\
\hline \multicolumn{7}{|l|}{$\mathrm{CC}, 10^{-3} \mathrm{~mm} 2 / \mathrm{kPa}$} \\
\hline & $p$ & - & $91 \pm 10$ & $95 \pm 7$ & $102 \pm 11^{4} t$ & $102 \pm 13^{*} \dagger$ \\
\hline & $\mathrm{NP}$ & - & $85 \pm 9$ & $85 \pm 10$ & $84 \pm 6$ & $83 \pm 8$ \\
\hline \multicolumn{7}{|l|}{ Hcit, volo } \\
\hline & $\mathbf{P}$ & - & $41 \pm 1$ & $39 \pm 1 * t$ & $38 \pm 1 * t$ & $35 \pm 2$ \\
\hline & $\mathrm{NP}$ & - & $40 \pm 1$ & $40 \pm 1$ & $39 \pm 1$ & $38 \pm 3$ \\
\hline \multicolumn{7}{|l|}{$n$} \\
\hline & $P$ & 9 & 9 & 9 & 9 & 8 \\
\hline & NP & 8 & 8 & 8 & 8 & 7 \\
\hline
\end{tabular}

Values are means \pm SD. $n$, no. of rats; $\Delta d$, antic diameter change during the cardiac cy de; $d$, end diastolic aortic diameter;

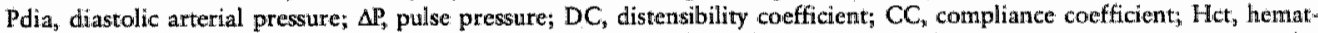
ocrit. Each variable was subjected to 2 -way analysis of variance by ranks (Friedman tesc). Pregnane (P) group: $P<0.005$ for $\Delta d ; P<0.05$ for Pdia, $C C$ and $D C_{j} P=0.0001$ for Hct. $P<0.05$ compared with day 4 by Wilcoxon's signed rank test, $+P<0.05$ compared with nonpregnant (NP) rats by Mann-Whitney U-test.

Toble 2 Aortic wall stracture in 20-ditys pregnant and nowpregnant rats

\begin{tabular}{lcc} 
& Pregnant & Nonpregnant \\
\hline CSA, $\mathrm{mm}^{2}$ & $0.37 \pm 0.02$ & $0.38 \pm 0.02$ \\
$M_{\mathrm{t}, \mathrm{mm}}$ & $69 \pm 5$ & $70 \pm 2$ \\
WSdia, kPa & $154 \pm 30$ & $176 \pm 13$ \\
Wsys, kPat & $325 \pm 58$ & $350 \pm 21$ \\
\hline
\end{tabular}

Values are means \pm SD. CSA, cross-sectional area of the aortic media at in situ pessel length; Mt, aortic media thickstess in diastole; WSdia, wall stress in diastolew WSsys, wall stress in systole. 
Figure 1

Percent changes, relatiwe to day 4 , in end-diastolic aortic diameter (d), arric diameter changes during the cardiac cycle (delta d), distensibility coefficient (dc), compliance coefficierit (cc), diastolic arterial blood pressure (Pdia) and pulse prefisure (delta $\mathrm{P}$ ) in pregnant $(\mathbf{\nabla})$ and nonpregnant $(\mathbf{C})$ rats. Values are means $\pm S E * P<0.05$ compared with day 4 by Wilcoxom's signed rank test.
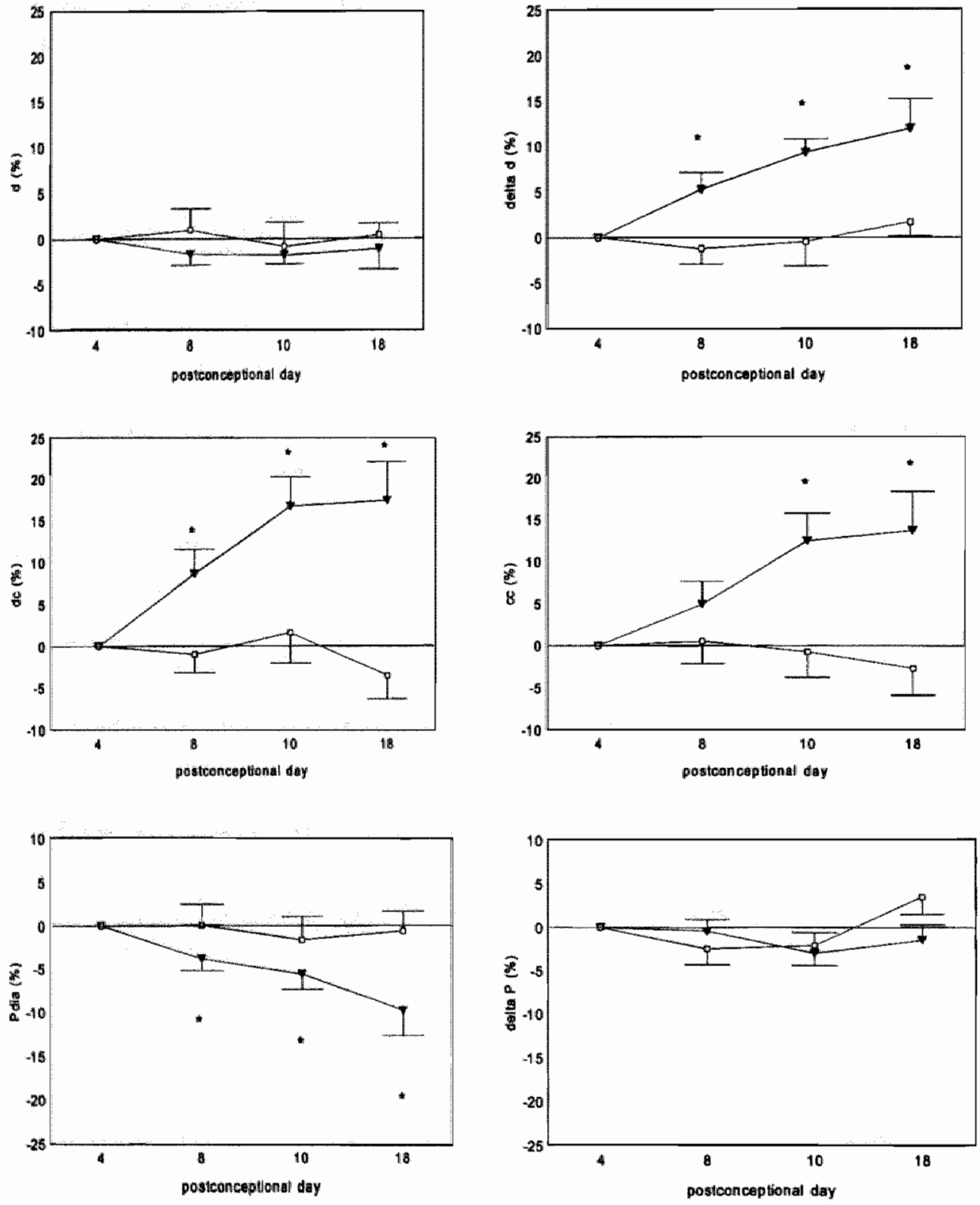
The main finding of our study is an increase in aortic compliance and distensibility between days 4 and 10 in rat pregnancy, while end-diastolic aortic diameter remains unchanged. Previous studies on the mechanical and structural properties of arteries and veins in pregnancy focused on late pregnancy. Aortic compliance and aortic diameter have increased in advanced human gestation (Hart 1986). Venous distensibility in the third trimester seems to be greater in pregnant than in nonpregnant women (Sakai 1994). In contrast, Hohmann et al found a decrease in venous distensibility in pregnant rats, possibly because this study was performed in isolated veins (Hohmann 1991). Studies on the changes in early pregnancy are scarce. Using a three-element Windkessel model, aortic compliance seems already increased at the end of the first trimester of human pregnancy (Hibbard 1994). Our study confirms the increased aortic compliance in late pregnancy, and adds to this information that these changes start in early pregnancy. An increase in arterial compliance can be caused by a decrease in pressure because of the anisotropic nature and nonuniform elastic composition of the walls of large arteries (Nichols 1990, Simon 1987). However, in our study, while Pdia further decreases after the 10 th day of pregnancy, CC plateaues. Therefore, it seems likely that factors other than the fall in blood pressure contribute to the increased arterial compliance in early pregnancy. To analyze the contribution of changes in structural wall properties to the change in aortic function observed by ultrasound wall tracking, we assessed the aortic wall structure by morphometry. We corrected histological findings with respect to media cross-sectional area for the changes that occurred during vessel isolation and took into account the in vivo measurement of diastolic lumen diameter to calculate media thickness. We were unable to find a change in the aortic media cross-sectional area and media thickness as a result of pregnancy. Thus, the observed functional changes are most likely not related to changes in vessel wall mass. However, others reported more subtle changes in ground substance and vessel metabolism (Conrad 1991), the importance of which for mechanics remains to be established.

The mechanisms reponsible for the hemodynamic changes in early pregnancy are still unclear. The changes in aortic mechanics which we observed are too limited to exert a major influence on the systemic hemodynamic characteristics during pregnancy. There are indications that, in early pregnancy, cardiac structure and function change in concert with changes in aortic function. Studies in human (Duvekot 1993), rat (Slangen 1996) and baboon (Phippard 1986) indicate a selective gradual increase in stroke volume, accompanied by a rise in left ventricular 
mass, left ventricular wall thickness and cardiac contractility (Buttrick 1987, Robson 1989). The apparently balanced changes in cardiac and aortic dynamics raise the question whether they are causally related or develop independently in response to some overall relaxing factor. To answer this question, it is important to keep in mind that in pregnancy, the compliance of the entire vascular bed may be increased (Davis 1989, Humphreys 1994). A larger left ventricular size (Hart 1985, Morton 1984) without increase in filling pressure (Davis 1989) in guinea pig pregnancy, can be interpreted as an effect of an increased cardiac compliance. Assuming the development of a higher overall vascular compliance in pregnancy, together with a lower total peripheral resistance, we postulate that one of the first effects of the changed vascular dynamics will be sodium and water retention (Duvekot 1993, Schrier 1987) to maintain vascular filling pressure. In the heart, the higher compliance results in a larger end-diastolic volume (Buttrick 1987). At unchanged or increased cardiac ejection fraction (Capeless 1989, Robson 1989), the former will contribute to the observed increase in SV in early pregnancy (Slangen 1996). A raised stroke volume gives rise to a higher shear stress (Zarins 1987). Although the vascular tone will already be lower due to an overall relaxation, the endothelium will respond to this raised shear stress by releasing relaxing agents such as nitric oxide and PGI2 (Bassenge 1988). These secondary effects serve to protect the vessel wall, as also suggested by the unchanged, or even slightly decreased, aortic wall stress observed in our study. We speculate that the rise in aortic compliance and distensibility, together with the decrease in total peripheral recistance, in early pregnancy ocrue to accomodate the increate in SV in pregnancy, thus preventing a rise in blood pressure and aortic arterial wall stress.

\footnotetext{
Ackmawledgments

The authors thank Gregorio Fazzi for technical assistance.

This research was supported by the Netherlands Heart Foumdation (NHS Grant 91_098) and by a grant from Serwier.
} 
Aortic distensibility and compliance in conscious pregrant rats 


\section{Chapter 4}

\section{Blood pressure and heart rate variability in early pregnancy in the rat}

Brigitte F.M. Slangen ${ }^{1}$, Iris C.M. Out ${ }^{\mathbb{1}}$, Ben J.A. Janssen ${ }^{2}$ and Louis L.H. Peeters $^{1}$

Departments of Obstetrics and Gynecology $y^{1}$ and Pharmacology ${ }^{2}$, University of Maastricht, $6200 \mathrm{MD}$ Maastricht, The Netherlands.

American Journal of Physiology, in press. 


\section{Abstract}

Changes in the autonomic control of the circulation may contribute to the maternal hemodynamic adaptation to early pregnancy. To evaluate this, we studied mean arterial pressure (MAP) and heart rate (HR) in chronically instrumented, conscious rats in early (days $4,6,8$ and 10 ) and late (day 18) pregnancy $(n=8)$ and in nonpregnant rats $(n=9)$. MAP and HR were recorded on a beat-to-beat basis and analyzed by spectral analysis. Spectral density power was calculated in low- (0.047-0.305 Hz), mid- (0.305-0.598 Hz), and high-frequency (0.598-1.494 $\mathrm{Hz}$ ) bands, which contain oscillations that are, amongst others, related to myogenic, sympathetic/vagal, and vagal/respiration-related influences, respectively. In addition, baroreceptor reflex sensitivity was determined from spontaneous variations in MAP and HR by a sequential time series method and by calculating the transfer gain between MAP and HR in the mid-frequency band.

Mean values of HR and MAP did not differ between the two groups on day 4. In the pregnant group, MAP fell gradually over days, while HR had significantly increased only on day 18. Overall variability in MAP and HR (expressed as coefficients of variation) did not change in the course of pregnancy. Baroreceptor reflex sensitivity did neither differ between the groups, nor did it change with advancing pregnancy. Spontaneous oscillations of MAP and HR at low, mid and high frequencies were not different between pregnant and nonpregnant rats on days 4 to 10 . On day 18 , spectral density power of MAP, but not of HR, in the high-frequency band had significantly increased, in pregnant rats only, most likely reflecting the increased impact of breathing on MAP fluctuations. We conclude that, with the methods employed, we could not discern any changes in baroreflex sensitivity and blood pressure and heart rate variability in pregnancy. This would imply that changes in autonomic activity do not contribute appreciably to the hemodynamic adaptations in early rat pregnancy. 


\section{Introduction}

Hemodynamic adaptation to early human as well as early rat pregnancy is characterized by a fall in blood pressure and total peripheral resistance, together with a rise in cardiac output and stroke volume (Duvekot 1993, Slangen 1996). This high-flow low-resistance circulation is accompanied by an elevated plasma renin activity (Conrad 1989, Duvekot 1993), suggesting that early pregnancy hemodynamics may be associated with a relative vascular underfill (Schrier 1987). However, in the rat, this relative vascular underfill does not trigger a rise in heart rate, although cardiac contractility increases (Buttrick 1987). This suggests that reflex regulation of heart rate changes during early pregnancy. Studies on baroreceptor reflex function and autonomic tone in pregnancy have revealed contradictory results. In human pregnancy neither catecholamine levels nor heart rate variability have changed (Barron 1986, Eneroth 1994) suggesting an unaltered sympathoadrenal function. On the other hand, autonomic function tests indicate a decreased parasympathetic responsiveness (Ekholm 1993, Ekholm 1994). An increase in baroreflex-mediated bradycardia in rat (Conrad 1992) and human pregnancy (Leduc 1991), as well as unchanged baroreflex control in rat pregnancy (Crandal』1990, Hines 1992) has been reported. These discrepancies might be due to differences in methodology, using autonomic function tests or pharmacological interventions (Conrad 1992, Crandall 1990, Ekholm 1993, Ekholm 1994, Hines 1992, Leduc 1991). In addition, most studies focused on late pregnancy (Ekholm 1992, Ekholm 1993, Eneroth 1994, Leduc 1991), or at the end of the first trimester (Ekholm 1994), when most of the hemodynamic adaptations are already fully developed (Duvekot 1993).

Recently, methods have been developed to assess autonomic influences and the cardiac baroreflex gain from computer analysis of spontaneously occurring changes in arterial blood pressure and heart rate (Bertinieri 1988, Cerutti 1991, Oosting 1997). These methods are attractive, since they provide information about cardiovascular control without pharmacological or mechanical manipulation of the baroreflex and the autonomic nervous system. Spectral analysis of beat-to-beat fluctuations in heart rate (HR) and mean arterial pressure (MAP) may provide insight into the sympathetic and parasympathetic influences on the cardiovascular system (Stauss 1996). Generally, two or more different frequency bands are identified. In conscious, unrestrained rats, it was demonstrated that spectral power of MAP in mid-frequencies $(0.27-0.74 \mathrm{~Hz})$ depends primarily on 
the activity of sympathetic nervous fibers. The baroreflex accounts for about $50 \%$ of this power (Cerutti 1994, Julien 1995). The spectral power of HR in mid-frequencies seems to be determined by vagal rather than sympathetic influences (Perlini 1995). Efferent vagal influences are responsible for a fraction of the high frequency $(0.75-3 \mathrm{~Hz})$ power of MAP and HR, but a large part of these fluctuations are due to the mechanical impact of breathing (Cerutti 1994, Janssen 1995, Perlini 1995). Low-frequency $(0.08-0.18 \mathrm{~Hz})$ oscillations of MAP result primarily from vasomotion (Janssen 1995, Julien 1995, Rizzoni 1995).

Baroreceptor reflex sensitivity can be determined from spontaneous variations in MAP and HR by a sequential time series method (Bertinieri 1988, Iellamo 1996, Oosting 1997) or by calculating the transfer gain between MAP and HR in defined frequency ranges (Cerutti 1994, Fritsch 1986, Robbe 1987).

Using the aforementioned techniques we evaluated whether changes in autonomic nervous activity or baroreceptor reflex sensitivity contribute to the hemodynamic adaptation in early pregnancy. To this end, we assessed HR and MAP on a beat-to-beat basis in chronically instrumented, conscious pregnant rats, along with 9 nonpregnant rats. Measurements were performed on days $4,6,8$, 10 and 18 of pregnancy since the majority of the hemodynamic adaptations in rat pregnancy takes place between days 4 and 10 of pregnancy (Slangen 1996). In addition, day 18 of pregnancy was included, since HR increases in late pregnancy (Slangen 1996).

\section{Materials and methods}

SURGICAL PROCEDURES Seventeen female Wistar rats (Winkelmann, Borchen, Germany) were studied, at the age of 3-4 months. All procedures were approved by the Institutional Animal. Care and Use Committee of the University of Limburg, Maastricht. Rats were kept on a 12h:12h light-dark cycle. Rats were mated with a male and presence of a sperm plug was defined as day one of pregnancy. On this day, surgery was performed aseptically. Under ketamine $(40 \mathrm{mg} / \mathrm{kg})$ and xylazine $(4 \mathrm{mg} / \mathrm{kg})$ anaesthesia, a polyethylene catheter $(0.28 \mathrm{~mm} \mathrm{ID}, 0.61 \mathrm{~mm}$ OD) was advanced into the abdominal aorta via the femoral artery, as described previously (Slangen 1996). The catheter was exteriorized between the scapulae, filled with heparinized saline $(5 \mathrm{IU} / \mathrm{ml})$ and plugged with a stainless steel pin. 
DATA ACQUISTTION AND ANALYSIS Measurements were performed on days 4, 6, 8, 10 and 18 or 19 of pregnancy ( $\mathrm{P}$ group; $\mathrm{n}=8$ ) and in nonpregnant rats (NP group; $\mathrm{n}=9$ ). On experimental days, the arterial catheter was comnected to a pressure transducer (Statham). The signal was sampled continuously at $1000 \mathrm{~Hz}$ and beatto-beat values were stored on disk for off-line analysis. Recordings were started after approximately 30 minutes, when the rat was resting quietly and stable tracings had been obtained for at least 15 minutes. Then, data sampling was performed for a period of 20-30 minutes. The beat-to-beat registration of MAP and HR was replayed on screen for visual inspection. Data segments with artifacts due to obstruction of the arterial catheter were eliminated. We calculated mean ( \pm SD) HR and MAP for the 20-30 min experimental period, as well as overall variability, expressed as the coefficients of variation (CV; standard deviation divided by mean times $100 \%$ ).

A. Tracings from an 8-days pregnant zat showing examples of recordings of HR and MAP. B: spectral power plots. of corresponsing, tracings. Low-frequency (LF; 0.047 to $0.305 \mathrm{~Hz}$ ), mid-frequency (MF; $0.305-0.598 \mathrm{~Hz}$ ), and high. frequency bands $\left(\mathrm{HF}_{3}, 0.598-1.494 \mathrm{~Hz}\right)$.
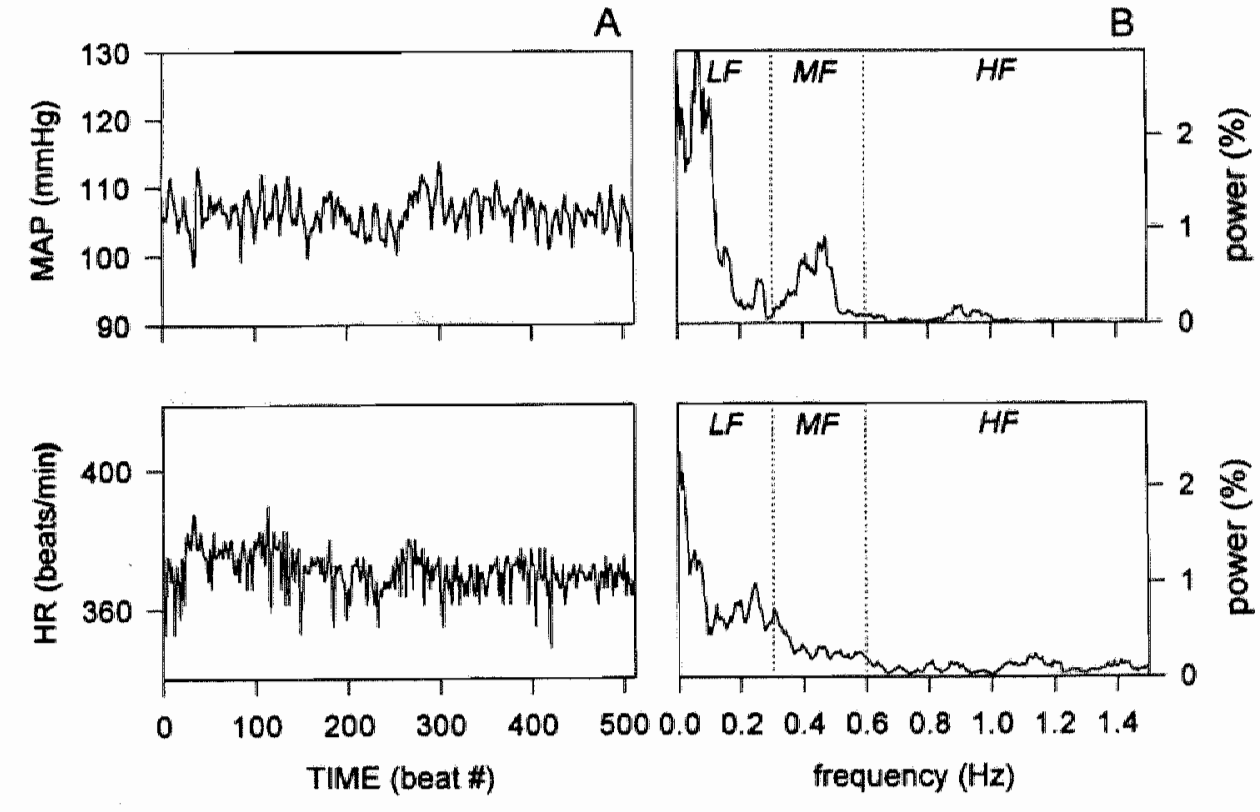
Two pregnant and two nonpregnant rats could not be studied on day 18 postsurgery, due to catheter failure.

SPECTRAL ANALYSIS Spectral density power of the various frequency components of MAP and HR was calculated using a fast Fourier transformation (Janssen 1995). For this analysis technique equidistantly sampled data are required. Therefore, the beat-to-beat datapoints were converted into equidistant time series using an algorithm that extracted from sequential $200-\mathrm{ms}$ time windows the maximum value of each parameter. The resulting $(5 \mathrm{~Hz})$ time series were divided into halfoverlapping sequential sets of 512 data points. Before calculation of spectral density power, each segment was subjected to linear trend removal and cosine tapering of the first and last 60 data points. From sections of 512 values, the power and frequency of every spectral component was calculated (figure 1). Three frequency bands were defined, as indicated in figure 1:1) a low-frequency band (LF: 0.047 to $0.305 \mathrm{~Hz}$ ) representing oscillations in MAP that primarily result from vasomotion (Janssen 1995, Julien 1995, Rizzoni 1995), 2) a mid-frequency band (MF: 0.305-0.598 Hz) containing oscillations in MAP predominantly resulting from sympathetic influences (Cerutti 1994, Julien 1995, Stauss 1996), and oscillations in HR that may stem from parasympathetic influences (Perlini 1995), and 3) a high-frequency band (HF: 0.598-1.494 Hz) containing oscillations in MAP that are associated with respiration (Cerutti 1994, Janssen 1995, Perlini 1995). Spectral power was calculated in each defined frequency band, relative to total spectral power, which equals SD2.

\section{CALCULATION OF BARORECEPTOR REFLEX SENSITIVTYY}

1. Time series method Baroreceptor reflex sensitivity was determined from spontaneous fluctuations in MAP and HR using a time series method recently validated for rats (Oosting 1997). In short, beat-to-beat values of MAP and pulse interval $(60000 / \mathrm{HR})$ were low-pass filtered using a 10 beats moving average function. The filtered signal was searched for ramps of decreasing or increasing MAP of 4 beats or more. When a ramp was found, then the slope between the MAP ramp and pulse interval change at 3,4 and 5 beats delay was determined. Baroreceptor reflex sensitivity (BRS) was calculated as the average value of these 3 slopes.

2. Transfer function analysis In addition, baroreceptor reflex sensitivity was calculated as the transfer gain between MAP and HR oscillations in the mid-frequency band (Cerutti 1994, Fritsch 1986, Robbe 1987). The basic assumption 
that underlies this approach is that the system linking MAP and HR responds linearly (Fritsch 1986). In addition to the transfer gain, this analysis enables the calculation of two other parameters: 1) a coherence function, which provides an assessment of the linear relationship between MAP and HR at each frequency. Coherence can have a value between 0 and 1 and is a frequency domain estimate of the degree that the HR variance can be explained by linear operation of the MAP variance. The coherence is 1 if no other variables than MAP affects the HR oscillations (Cerutti 1994). 2) a phase function, which represents the temporal relationship between the MAP and HR signals in the frequency domain. The phase value indicates the portion of the period corresponding to the frequency $f_{\text {, }}$ of which the output signal HR is delayed relative to the input signal MAP. A positive phase indicates that the HR oscillations at frequency $f$ precede those of MAP. Finally, for each rat, the results obtained from these analysis techniques were averaged over the sequential data segments to reduce the variance.

STATISTICAL ANALYSIS We used nonparametric analysis of wariance for repeated measures (Friedman Test) to assess whether significant changes occurred within each group. If so, Wilcoxon's signed rank test was used for specific between-periods comparisons. Day 4 was selected as the reference day because the pregnant and nonpregnant groups were comparable on that day (Mann-Whitney U-test). Differences between the pregnant and nonpregnant groups were analyzed by Mann-Whitney U-test. Data are expressed as means \pm SD, unless stated otherwise. Differences were considered significant when the $\mathrm{P}$ was less than 0.05 .

\section{Results}

STEADY STATE VALUES OF HEMODYNAMIC PARAMETERS (TABLE 1) Table 1 lists mean values of, and overall variability in MAP and HR obtained during the 20-30 min recording sessions on the experimental days. Mean values of $H R$ and MAP did not differ between the two groups on day 4 . In the pregnant group, MAP decreased gradually from day 6 of pregnancy onward (from $125 \pm 12 \mathrm{mmHg}$ by day 4 to $101 \pm 10 \mathrm{mmHg}$ by day 18 ). HR had only increased in late pregnancy (from $373 \pm 21$ beats/min by day 4 to $408 \pm 24$ beats/min by day 18 ). In the nonpregnant group no significant changes were observed over time. The overall vari- 


\section{Chapter 4}

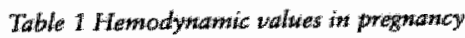

\begin{tabular}{|c|c|c|c|c|c|c|}
\hline & & day 4 & $d a y y^{6}$ & $d x y \&$ & day 10 & day 18 \\
\hline \multicolumn{7}{|l|}{$\mathrm{HK}$, beats/min } \\
\hline & $p$ & $373 \pm 21$ & $375 \pm 9$ & $377 \pm 10$ & $374 \pm 16$ & $408 \pm 24$ \\
\hline & NP & $358 \pm 21$ & $369 \pm 26$ & $365=21$ & $359 \pm 11$ & $368 \pm 25$ \\
\hline \multicolumn{7}{|l|}{ MAP, mmHg } \\
\hline & $\mathrm{p}$ & $\begin{array}{l}125 \pm 12 \\
121 \pm 8\end{array}$ & $\begin{array}{l}112 \pm 8^{*} \\
112 \pm 7\end{array}$ & $\begin{array}{l}107 \pm 9 \\
109 \pm 3\end{array}$ & $\begin{array}{l}110 \pm 7 \\
113 \pm 6\end{array}$ & $\begin{array}{l}101 \pm 10 \\
117 \pm 7\end{array}$ \\
\hline \multicolumn{7}{|l|}{ CV of $\mathrm{HR}_{p} \%$} \\
\hline & $P$ & $4.1=1.4$ & $3.9 \pm 1.2$ & $4.2 \pm 1.2$ & $3.6 \div 0.5$ & $3.2 \pm 0.5$ \\
\hline & $\mathrm{NP}$ & $4.6 \pm 1.3$ & $5.0 \pm 2.0$ & $3.7 \pm 0.8$ & $4.2 \pm 1.4$ & $4.4 \pm 1.1$ \\
\hline \multicolumn{7}{|l|}{$\mathrm{CV}$ of $\mathrm{MAP}, \%$} \\
\hline & $\mathrm{p}$ & $3.7 \pm 0.7$ & $4.1 \pm 0.9$ & $3.8 \pm 0.4$ & $4.0 \div 0.4$ & $3.8 \pm 1.2$ \\
\hline & NP & $3.6 \pm 0.5$ & $3.5 \pm 0.5$ & $3.3 \pm 0.6$ & $3.6 \pm 1.0$ & $3.7 \pm 1.0$ \\
\hline \multicolumn{7}{|l|}{$n$} \\
\hline & $\begin{array}{l}P \\
M P\end{array}$ & $\begin{array}{l}8 \\
9\end{array}$ & $\begin{array}{l}8 \\
9\end{array}$ & 8 & 8 & 6 \\
\hline & 14 & 7 & & & & \\
\hline
\end{tabular}

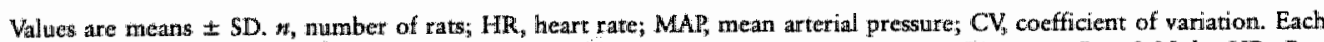
variable was subjected to 2 -way analysis of variance by ranks (Friedman test): pregnant (P) group: $P<0.05$ for HR; $P<$ 0.01 for MAP * $P<0.05$ compared with day 4 by Wilcoxon's signed ranik test. $+P<0.05$ compared with nonpregnant (NP) rats by Mann Whitney U test.

Table 2 Baroreceptor reflex sensitivity in pregnawcy

day $4 \quad$ day $6 \quad$ day $8 \quad$ day $10 \quad$ day 18

\section{Time series method}

BRS, ms/mm $/ \mathrm{mig}$

$$
\begin{array}{llllll}
\mathrm{P} & 0.49 \pm 0.23 & 0.56 \pm 0.19 & 0.55 \pm 0.14 & 0.46 \pm 0.12 & 0.51 \pm 0.21 \\
\mathrm{NP} & 0.67 \pm 0.36 & 0.66 \pm 0.30 & 0.67 \pm 0.23 & 0.74 \pm 0.35 & 0.71 \pm 0.40
\end{array}
$$

Transfer function analysis

Transter gain, oy

$\begin{array}{llllll}\text { NP } & 2.60 \pm 0.76 & 2.98 \pm 0.76 & 3.14 \pm 0.71 & 2.75 \pm 0.41 & 4.20 \pm 1.25 \\ \text { NP } & 2.94 \pm 0.89 & 3.61 \pm 1.84 & 3.10 \pm 1.34 & 3.16 \pm 0.75 & 4.17 \pm 1.63\end{array}$

Phasise, is

$\begin{array}{llllll}\text { P. } & 0.65 \pm 0.18 & 0.70 \pm 0.15 & 0.56 \pm 0.36 & 0.56 \pm 0.20 & 0.66 \pm 0.23 \\ \text { NP } & 0.70 \pm 0.31 & 0.71 \pm 0.27 & 0.79 \pm 0.28 & 0.76 \pm 0.23 & 0.47 \pm 0.26\end{array}$

Coherence

$\begin{array}{lllllll}\text { P } & 0.70 \pm 0.03 & 0.71 \pm 0.02 & 0.70 \pm 0.06 & 0.69 \pm 0.04 & 0.71 \pm 0.07 \\ \text { NP } & 0.69 \pm 0.05 & 0.73 \pm 0.04 & 0.74 \pm 0.04 & 0.70 \pm 0.07 & 0.66 \pm 0.09\end{array}$

n

$\begin{array}{llllll}\text { p } & 8 & 8 & 8 & 8 & 6 \\ N P & 9 & 9 & 9 & 9 & 7\end{array}$

Values are means \pm SD. n, number of rats; BRS, baroreflex sersitivity. Transfer gain, phase and coherence benween MAP and $\mathbb{U} R$ in the mid-frequency band $(0.305-0.598 \mathrm{~Hz})$. Each variable was subjected to 2 -way analysis of variance by ranks (Friedman test). 
Figure 2.

A: Average spectra of mean arterial pressure in pregnant (thick line) and nonpregnant (dhin line) rats on days 4,6 , 8,10 and 18 of pregnancy or postsurgery. B: Average spectra of heart rate in pregnant (thick line) and nonpregnant (thin line) rats on days $4,6,8,10$ and 18 of pregnancy or postsurgery

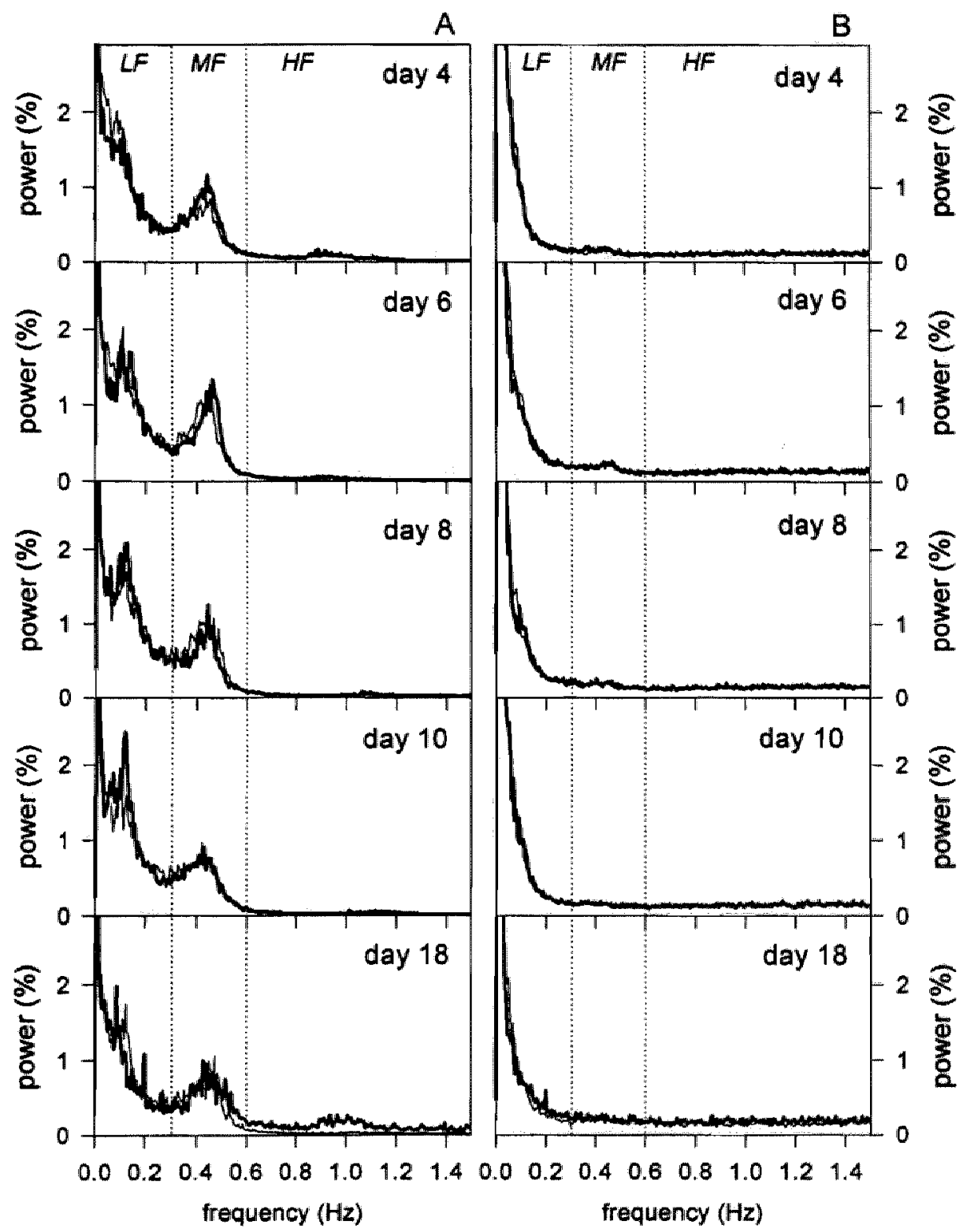


ability of MAP and HR, expressed as CV, remained unchanged throughout the experimental period in both groups.

SPECTRAL ANALYSIS (FIGURE 2) Average spectra of MAP and HR obtained in pregnant and nompregnant rats are given in figure 2 . In both groups, the spectral power of MAP was lowest in the HF, intermediate in the MF and highest in the LF band. The LF and MF bands contained clear peaks centered around $\sim 0.13$ and $\sim 0.45 \mathrm{~Hz}$, respectively. Spectral power of $\mathrm{HR}$ was low in both the HF and MF bands. Most variability was observed in the LF band. No differences were observed between pregnant and nonpregnant rats, except for day 18. MAP spectra, but not HR spectra, obtained in 18 days pregnant rats showed increased power in the HF band. No changes were observed in the nonpregnant group.

\section{BARORECEPTOR REFLEX SENSITIVTTY (TABLE 2)}

1. Time series method In pregnant rats (day 4 of pregnancy) the BRS index, calculated from spontaneous fluctuations in pressure, was $0.49 \pm 0.23 \mathrm{~ms} / \mathrm{mmHg}$, and did not change in the course of pregnancy. Values for nonpregnant rats changed little with time and were not different from the pregnant group (Table 2). These BRS values are comparable to those previously reported for male Wistar Kyoto rats (Oosting 1997).

2. Transfer gain Average coherence, gain and phase spectra are summarized in Table 2 and did not differ between groups. No changes were observed in the course of pregnancy. Coherence between MAP and HR was $>0.5 \mathrm{~Hz}$ in all frequency bands. Phase estimates were positive in all frequency bands indicating that HR oscillations at these frequencies precede those of MAP.

\section{Discussion}

The purpose of this study was to investigate whether autonomic control of MAP and $H R$, as assessed by their spontaneous variability, changes during early pregnancy in rats. Our results demonstrate that MAP and HR variability and baroreceptor reflex sensitivity do not change during early pregnancy in the rat. MAP has decreased by day 10 of pregnancy compared to the control group, whereas HR has increased by day 18 of pregnancy. These findings are in agreement with previous studies (Conrad 1992, Slangen 1996). Overall variability (CV) of MAP and 
HR did not change with advancing pregnancy and was comparable in pregnant and nonpregnant rats. Also, the LF, MF and HF components of MAP spectra did not change in early pregnancy. These results indicate unchanged vasomotion, sympathetic and respiratory influences on MAP during pregnancy, respectively. In addition, unchanged LF, MF and HF components of HR spectra suggest that (para)sympathetic and respiratory influences on $\mathrm{HR}$ remain unaltered during pregnancy in rats, respectively. The only change observed in our study was an increase in the power of the HF band of the MAP spectrum on day 18 of pregnancy. As MAP oscillations in this frequency band are mainlly related to respiration (Julien 1995, Perlini 1995), we speculate that in our pregnant rats elevated intrathoracic pressure, caused by the rapidly growing conceptus in late pregnan$\mathrm{cy}$, may have magnified the impact of breathing on pressure oscillations. The breathing frequency did not change, as evidenced by the fact that the HF band remained centered around $-1 \mathrm{~Hz}$. This does not exclude the possibility that breathing excursions may have become more profound.

Our present findings in late-pregnant rats are in agreement with those of Barron et al. in humans (Barron 1986). Their observation of comparable catecholamine levels in late-pregnant and postpartum populations suggest that pregnancy does not alter sympathoadrenal function (Barron 1986). However, hormone levels may not be conclusive, and interpretation of plasma catecholamine levels is complicated by methodological pitfalls in sample collection and measurement (Hjemdahl 1996). In other studies in human pregnancy, using cardiovascular autonomic function tests, (Ekholm 1993, Ekholm 1994) or spectrall analysis of HR (Ekholm 1992, Eneroth 1994), pregnancy seemed to be associated with a decreased parasympathetic responsiveness, from the first trimester onward (Ekholm 1994), persisting in the second trimester (Ekholm 1992, Ekholm 1993, Ekholm 1994), and returning to prepregnant levels in the third trimester (Ekholm 1994, Eneroth 1994). It was suggested that the fall in parasympathetic tone accounted for the known increase in resting HR in pregnancy (Ekholm 1992). However, although basal HR was elevated at day 18 of rat pregnancy in our study, we found no alterations in HR variability. Thus, our data in rats do not support a lower parasympathetic responsiveness of $\mathrm{HR}$ and add to the existing data that no changes can be observed in early pregnancy.

Baroreceptor reflex sensitivity, determined by the time series method and by the transfer gain, remained unaltered during pregnancy. In early pregnancy, basal MAP decreased while HR remained unchanged. This may reflect a leftward shift 
of the MAP-HR curve due to a downward resetting of the baroreflex. Only in late pregnancy had HR increased, however, without change in baroreceptor reflex sensitivity. Our results on the cardiac baroreflex in late pregnancy are in agreement with previous studies in late-pregnant rats (Crandall 1990, Hines 1992). Hines et al. demonstrated similar HR responses to increments in blood pressure after phenylephrine administration in late-pregnant and virgin rats. It was also observed that sino-aortic denervation had similar effects on baseline blood pressure in pregnant and virgin rats (Hines 1992). Others have also reported unchanged baroreflex sensitivity during late pregnancy in studies where isolated carotid sinus manipulation was employed to vary the reflex response (Humphreys 1974, Humphreys 1977). In addition, we observed no changes in the cardiac baroreflex in early pregnancy, when most of the hemodynamic changes develop. In contrast, other authors have reported an increase in baroreflex sensitivity to infusions of vasoactive agents in pregnant women at term and in late-pregnant rats (Conrad 1992, Leduc 1991). Reasons for the discrepancies regarding gestational effects on autonomic function and baroreflex sensitivity of $\mathrm{HR}$ are unclear, but may relate to species difference, effects of anesthesia, variations in experimental design and altered sensitivity to vasoactive agents. Our study was performed in chronically instrumented animals, fully recovered from surgery, and we examined autonomic function in the basal, unstimulated state in the period in which most of the hemodynamic adaptations are achieved (early pregnancy, Slangen 1996). It would be most reliable to measure sympathetic nervous activity using direct methods such as measurement of sympathetic nerve activity, however this method is not suitable for long-term recordings in the same animal. We preferred the use of spectral analysis and BRS measurements from spontaneous variations in blood pressure and heart rate for several reasons. First, the administration of vaso-active substances to compare changes in heart rate as a consequence of imposed blood pressure changes, could influence the baroreceptor reflex by a direct effect on receptor or effector sites. Second, the influence of these substances on the conceptus itself, and the changed sensitivity of the vessel wall to vaso-active substances in pregnancy (Conrad 1992), may invalidate comparisons with nonpregnant rats. Third, autonomic nervous function and BRS are measured in the physiological blood pressure range. Fourth, these methods do not impose any extra stress on the animals. However, we cannot exclude the possibility that baroreceptor control of sympathetic nerve activity to peripheral organs is modified. Interestingly, it has been demonstrated by Crandall et al. that 
renal sympathetic outflow in response to a hypotensive challenge is impaired in late-pregnant rats (Crandall 1990).

The calculation of the transfer function between pressure and heart rate to assess baroreceptor reflex sensitivity has been validated in humans (Robbe 1987). In the present study in rats, however, the phase relation between MAP and HR in the MF range was always positive. This indicates that HR oscillations at this frequency range are probably responsible for the oscillations in MAP. Thus, the transfer gain between MAP and HR in the MF range is not a suitable index for baroreceptor reflex sensitivity in the rat (Oosting 1997). Recent studies have indicated that oscillations lower than those in the MF range might be responsible for the baroreflex changes (Di Rienzo 1996).

In summary, the present data suggest unaltered activity of the autonomic regulation of blood pressure and heart rate during early pregnancy in rats. We speculate that the high-flow low-resistance circulation in early pregnancy, with its higher demand for intravascular fluid develops so slow, that the concomitant volume retention is adequate at any time to preserve the mean circulatory filling pressure and with it, venous return. This low rate of change with time may be even of vital importance for pregnancy as a too rapid rate of change carries the risk of a stress response with a higher sympathetic tone and with it a compromise of the blood supply to the implantation sites.

\section{Acknowledgenent}

We would like to thank Jan Oosting (Department of Pharmacology, University of Maastricht, Maustricht, The Netherlandsis) for help with data analysis. 


\section{Chapter 5}

\section{The mechanism of volume adaptation in early rat pregnancy}

Carla M. Verkeste ${ }^{1}$, Brigitte F.M. Slangen ${ }^{1}$, Marie-Louise Dubelaar ${ }^{1}$, Iris C.M. Out ${ }^{1}$, Bernard K. van Kreel ${ }^{2}$, Louis L.H. Peeters ${ }^{4}$.

Department of Obstetrics and Gynecology, University of Maastricht, and department of Clinical Chemistry, University Hospital Maastricht ${ }^{2}$, Maastricht, The Netherlands.

Submitted 


\section{Abstract}

The objective of the present study was to determine whether the increase in PV during pregnancy is established by fluid retention or by a shift within the extracellular fluid volume (ECFV) from the interstitium towards the intravascular compartment. To this end, we measured simultaneously total body water (TBW), ECFV and plasma volume (PV) together with the hematocrit (Hct) and plasma osmolality, 4,8 and 12 days postsurgery in chronically instrumented pregnant and nonpregnant rats. The pregnant rats were instrumented with a catheter in the femoral artery on day 1 postconception. In the nonpregnant group neither TBW nor ECFV and PV had changed consistently on days 8 and 12 postsurgery relative to day 4. In contrast, in the pregnant animals TBW, ECFV and PV had increased by $16 \%, 24 \%$ and $20 \%$, respectively, by day 12 relative to day 4 . In order to evaluate whether PV had increased in concert with an overall rise in TBW or as a result of a fluid shift at the cost of the interstitial volume, we calculated the relative size of each fluid compartment on 3 consecutive measurement sessions. TBW (expressed as a percentage of maternal weight), and the percentage of TBW attributed to ECFV and PV, respectively, was not different between day 12 and day 4 in the nonpregnant rats. In the pregnant animals, TBW (\%MW) was slightly higher on day 12 as compared to day 4 . Meanwhile, neither ECFV (\%TBW) nor PV (\%ECFV) had changed. Finally, in the nonpregnant group Hct had not changed while in the pregnant animals Hct was $10 \%$ lower on days 8 and 12 as compared to day 4 . Plasma osmolality did not change consistently in either group during the course of the experimental period.

The gradual synchronous increase in all fluid compartments, without consistent change in their relative distribution suggests that in normal rat pregnancy PV expansion is achieved by fluid retention rather than by redistribution of the ECFV. 


\section{Introduction}

Previously, it has been shown that pregnancies complicated by fetal growth restriction and/or preeclampsia are characterized by subnormal expansion of the plasma volume (PV) compartment during pregnancy (Duvekot 1995, Hays 1985). On the basis of this observation it is generally assumed that PV expansion is important for normal pregnancy development. Serial measurement of a number of hemodynamic and volume-related variables in human (Duvekot 1994, Schrier 1991), baboon (Phippard 1986) and rat pregnancy (Slangen 1996) has provided indirect evidence for the concept that the first adaptive change in hemodynamics in pregnancy, is generalized vascular relaxation giving rise to the institution of a high-flow low-resistance circulation and PV expansion. The concomitant rise in plasma renin activity (Conrad 1989a, Wilson 1980) and the decreased threshold for vasopressin release (Davison 1988, Dürr 1981), indicate that the consequence of the high-flow low-resistance circulation is a relatively underfilled vascular bed. The latter, in turn, can be expected to induce sodium and volume retention (Hytten 1966). In late-pregnant rats on a sodium-restricted diet, hematocrit does not decrease, suggesting that the normally occurring plasma volume expansion is achieved by sodium and water retention (Ganguli 1970, Kirksey 1962, Pike 1976). In contrast, Baylis and Munger (Baylis 1990) reported a normal increase in PV in early-pregnant rats subjected to complete sodium deprivation. The latter suggests that PV expansion in early pregnancy may be achieved by a fluid shift from the interstitium to the intravascular compartment. Since the evidence for both concepts is based on quantification of only PV or Hct, the experimental evidence for either concept of PV expansion is still lacking. The objective of the present study was to determine which one of these two mechanisms is operative in early pregnancy. Therefore, we measured simultaneously and serially total body water (TBW), extracellular volume (ECFV) and PV together with hematocrit $(\mathrm{Hct})$ and plasma osmolality in chronically instrumented pregnant and nonpregnant rats. 


\section{Materials and methods}

ANMAL PREPARATTON Thirteen Wistar rats (Charles River, Sulzfeld, Germany) were used, at the age of 3-4 months. In addition, a separate group of 10 Wistar rats, at the age of 1 year, were used for blood donation. All facilities and procedures were approved by the Institutions Animal Care and Use Committee of the University of Maastricht, Maastricht. The animals were allowed one week of acclimatization to the centralized experimental animals facilities. This included a $12 \mathrm{~h} / 12 \mathrm{~h}$ light/dark cycle, and free access to standard rat chow (salt intake \pm 70 $\mathrm{mg} /$ day)(Hope farms, Woerden, The Netherlands) and acidified water. After one week 13 animals were mated with a fertile male. The presence of a sperm plug in the cage, which was confirmed in 8 of these animals was defined as day one of pregnancy. The animals that did not conceive were allocated to the non-pregnant group (NP, $\mathrm{n}=5$ ). All 13 animals underwent surgery using aseptic techniques as described previously (Slangen 1996). In the pregnant animals (P) surgery was always performed on day 1 of pregnancy. Prior to surgery general anesthesia was induced using ketamin $(50 \mathrm{mg} / \mathrm{kg})$ and xylazin $(5 \mathrm{mg} / \mathrm{kg})$, intramuscularly. A polyethylene catheter $(\mathrm{OD} / \mathrm{ID}=0.61 \mathrm{~mm} / 0.28 \mathrm{~mm}$, heat-sealed into a piece of $\mathrm{OD} / \mathrm{ID}$ $=0.96 / 0.58 \mathrm{~mm})$, filled with heparinized saline $(5 \mathrm{IU} / \mathrm{ml} 0.9 \% \mathrm{NaCl})$ was inserted into a femoral artery and advanced $\pm 4 \mathrm{~cm}$ resulting in the positioning of the catheter tip within the abdominal aorta just below the renal arteries. Another polyethylene catheter $(\mathrm{OD}: \mathrm{ID}=0.96: 0.58 \mathrm{~mm}$ melted with ether to a piece of silicon) was inserted into the ipsilateral femoral vein and advanced $\pm 4 \mathrm{~cm}$ into the inferior vena cava. The catheters were closed with a metal pin, tunneled subcutaneously to the neck and fixed between the shoulder blades.

EXPERIMENTAL DESIGN TBW, ECFV and PV were determined with deuteriumoxide $\left(\mathrm{D}_{2} \mathrm{O}\right)$ (Van Kreel 1996), sodium bromide ( $\mathrm{NaBr}$ ) (Van Kreel 1994) and Evans blue (EB) (Gibson 1937), respectively, on the basis of their degree of dilution in their distribution spaces. These compounds tend to accumulate when used repeatedly in one animal, particularly when the intervals are shorter than 48 hours. This was noted in a preceding pilot study in 3 nonpregnant rats. On the basis of the results of this pilot study, we concluded that a wash-out period of at least 4 days should be adopted to obtain reliable consecutive data points and also, that the measurement error increased progressively when more than three consecutive measurements were performed in one animal. To describe the entire interval of 
early pregnancy (from day 4 to 12) we decided to use an interval of 4 days between measurements and to limit the number of measurements to 3 in each animal. Therefore, the animals were measured 4,8 and 12 days postconception (P) or postsurgery (NP). At the time of each measurement the animal was placed in an experimental cage. The catheter ends between the shoulder blades were connected to a polyethylene extension catheter (OD:ID $=0.61 \mathrm{~mm}: 0.28 \mathrm{~mm}$ ) which allowed sampling and infusion without manipulation of the awake animal. One hour later the experiments were started by blood sampling $( \pm 0.7 \mathrm{ml}$ ) for later measurement of the baseline concentrations of $\mathrm{D}_{2} \mathrm{O}, \mathrm{NaBr}, \mathrm{EB}, \mathrm{Hct}$ (microcapillary method) and plasma osmolality (mosmol, Osmomat 030-D, Gonotec). When sampling was completed, a weighed cocktail ( $\pm 0.25 \mathrm{gram}$ ) of $\mathrm{D}_{2} \mathrm{O}( \pm 0.1 \mathrm{ml}$ ), $\mathrm{NaBr}$ ( $\pm 1.54 \mathrm{mg}$ ) and $\mathrm{EB}( \pm 0.1 \mathrm{ml}, 2 \%$ ) was injected intravenously as follows. The syringe with the cocktail was connected to the extended venous catheter, approximately $0.75 \mathrm{ml}$ of blood was withdrawn and mixed with the cocktail in the syringe. Than the $\pm 1 \mathrm{ml}$ blood/cocktail mixture was injected into the animal. To ensure that the entire cocktail was administered the syringe was slowly refilled with blood and reinjected a total of four times. The entire injection procedure lasted \pm 1.5 minutes. The catheter was flushed with saline. The amount of flushing was kept to a minimum (dead space $+0.1 \mathrm{ml}$ ) in order to avoid interference with the existing distribution of fluid over the various compartments in the animal. Ten minutes after completion of the first blood/cocktail injection the serum concentration of $\mathrm{D}_{2} \mathrm{O}, \mathrm{NaBr}$ and $\mathrm{EB}$ can be considered to have reached steadystate in their distribution compartments. This is based on repeated observations in the 3 nonpregnant animals evaluated during the preceding pilot study. Therefore, at that time a second heparinized blood sample of $0.7 \mathrm{ml}$ was collected for later measurement of the $\mathrm{D}_{2} \mathrm{O}, \mathrm{NaBr}$ and $\mathrm{EB}$ concentrations. After completion of this second sampling the animals received a transfusion of $1.4 \mathrm{ml}$ heparinized blood from a nonpregnant donor rat obtained by cardiac puncture. Ten minutes later the catheter extensions were disconnected, the catheters were flushed and closed with a metal pin and the animals were returned to their permanent cages. The experiments were always performed between $9.00 \mathrm{a} . \mathrm{m}$. and 2.00 p.m.. After completion of the experiment on day 12 the animals were sacrificed by $\mathrm{CO}_{2}$ inhalation. Littersize, fetal and placental weights and fetal viability were determined to exclude interference with normal pregnancy development. TBW, ECFV and PV were calculated from the degree of dilution of $\mathrm{D}_{2} \mathrm{O}, \mathrm{NaBr}$ and $\mathrm{EB}$, respectively, after correction for baseline concentrations. 
STATISTICS Data are presented as medians with range. Two-way analysis of variance by ranks (Friedman Test) was used. Wilcoxon Signed Rank Test was subsequently employed to test for changes relative to day 4 within the groups. A p value of less than 0.05 (two-sided) was considered significant.

\section{Results}

Maternal weight (MW) at day 4 was 250 and 208 grams in the NP and P group, respectively (table 1) and only increased in the $\mathrm{P}$ group in the course of the experimental period. Littersize and viability as well as fetal and placental weights were comparable to previous observations in pregnant rats (Hebel 1986, Miller 1980). In the NP group neither TBW nor ECFV and PV had changed consistently on days 8 and 12 relative to day 4 . In contrast, in the P group, TBW, ECFV and PV increased in the course of early pregnancy and had significantly increased by day 12 . In the P group the Hct on days 8 and 12 was approximately $10 \%$ lower than that on day 4 . In neither group plasma osmolality had changed consistently in the course of the experimental period.

In order to evaluate whether PV had increased in conjunction with an overall rise in TBW or as a results of a fluid shift at the cost of the interstitial volume, we calculated the relative size of each fluid compartment at consecutive measurement sessions (table 1). In neither group TBW (\%MW), ECFV (\%TBW) and PV (\%ECFV) had changed consistently in the course of the experimental period. Interstitial volume (ISFV, ECFV-PV) was calculated for each group and together with PV and TBW depicted in figure 1. In the NP animals ISFV had changed inconsistently. In contrast, in the P group, ISFV had increased in concert with the increase in PV and TBW. 


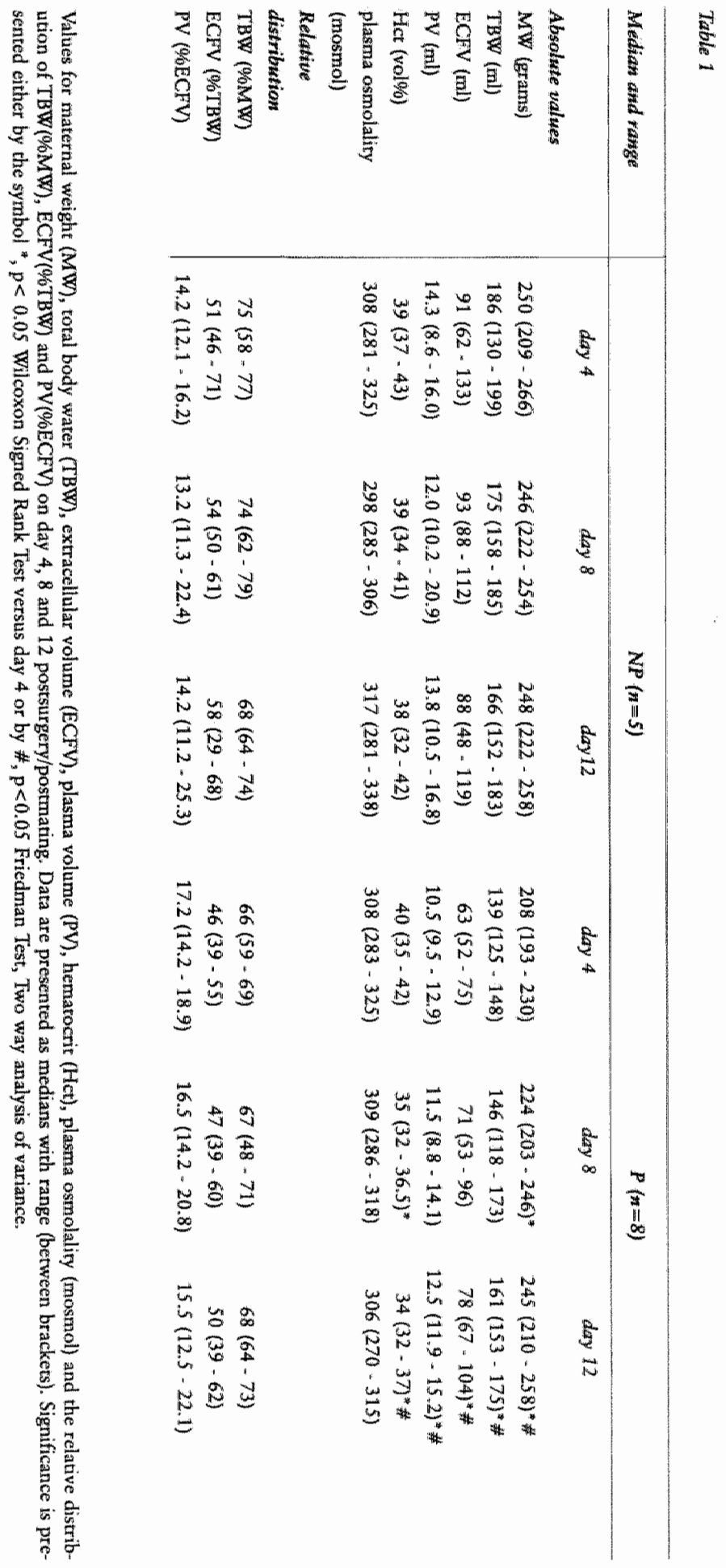




\section{Chapter 5}

Higure 1

PV, 15FV and TBW on days 4,8 and 12 postsurgery (n m) or postmating (n-8). Datais presented as medians together with 75 th and 25 th percentile. Significance is presented by the symbol ", $p<0.05$ Friedman analysis.
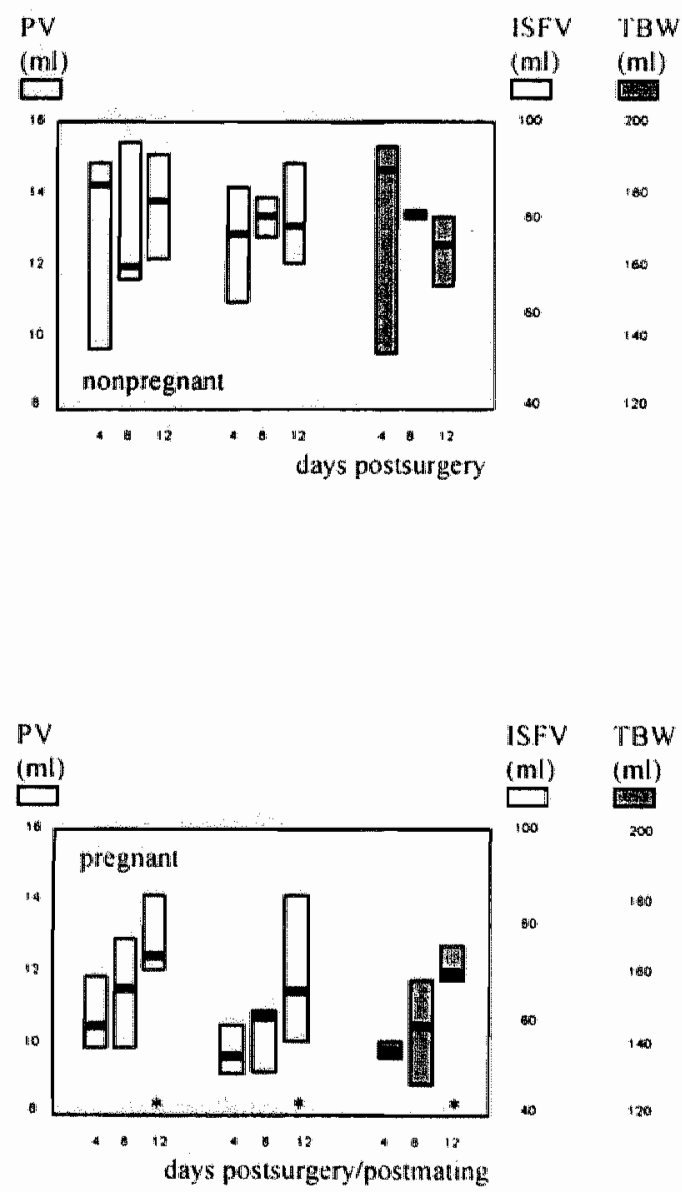


\section{Discussion}

The objective of the present study was to determine whether the increase in PV during pregnancy is established by fluid retention or by a fluid shift within the ECFV from the interstitium towards the intravascular compartment. To this end, we measured simultaneously TBW, ECFV, PV together with the Hct and plasma osmolality, in chronically instrumented pregnant and nonpregnant rats. The indicators used in the present study, $\mathrm{D}_{2} \mathrm{O}, \mathrm{NaBr}$ and $\mathrm{EB}$ have a measurement error of $2 \%, 5 \%$ and $5 \%$, respectively (Thomas 1991, Van Kreel 1996, Van Kreel 1994, Zipf 1955). When ISFV is calculated from subtracting PV from the ECFV the error in this parameter will be equal to the sum of the 2 contributors, namely $\pm 7 \%$. In the present study, the baseline values observed for TBW, ECFV and PV were within the normal range described previously for rats (Atherton 1982b, Barron 1987, Culebras 1977, Fowler 1986, Gilányi 1983, Lesser 1980, Lyons 1993, Sanz 1990). To ensure that the sensitivity of the applied method is sufficient to distinguish between the 2 possible mechanisms of PV expansion, we estimated the hypothetical change in the ECFV, PV and TBW for both options. If the rise in $\mathrm{PV}$ in early pregnancy $( \pm 25 \%$ )(Atherton 1982 , Barron 1987 , Gilányi 1983 , Baylis 1979/1980) would entirely result from a fluid shift at the cost of the ISFV, the $25 \%$ increase in PV would be paralleled by a $\pm 4 \%$ decrease in ISFV. In that case the change in ISFV would not be detected as the magnitude of the change in this parameter is smaller than the measurement error of $7 \%$. Discrimination between either a fluid shift or fluid retention in order to establish PV expansion, should therefore be based upon whether ECFV and TBW increase in concert with PV. In this study we observed a consistent increase in both ECFV $(+24 \%)$ and TBW $(+16 \%)$ by day 12 of pregnancy. The latter suggests that in normal rat pregnancy $\mathrm{PV}$ expansion is achieved by fluid retention rather than by a shift within the ECFV. Our results are in agreement with those obtained by other authors (Ganguli 1970, Kirksey 1962, Pike 1976) who observed a lack of an increase in blood volume after sodium deprivation. These results are not necessarily conflicting with those of Baylis and Munger (Baylis 1990), since the mechanism of plasma volume expansion might differ between early and late pregnant rats. Sodium deprivation in late pregnancy leads to compromised fetal development which might indirectly lead to a reduction in plasma volume. Baylis and Munger (Baylis 1990) observed normal PV expansion in early-pregnant rats subjected to zero sodium intake. It should be emphasized, though that the latter 
observation does not exclude the possibility that TBW and ECFV may have increased e.g. by water retention triggered by non-osmotic vasopressin release, in order to compensate for the inability to raise sodium retention. Water exchange in the capillary bed depends on the capillary and interstitial hydrostatic pressures on the one hand and its counterpart, the capillary and interstitial oncotic pressures on the other hand. These so-called Starling forces preserve a continuous balance of fluid movement within the capillary bed. Under normal physiological conditions the sum of ISFV and PV, ECFV, is maintained within narrow limits which are controlled by baro- and volume receptors distributed over the entire cardiovascular system (Moe 1991). In the presence of a high-flow low-resistance circulation a higher circulating volume is required. After activation of the volume sensors the ECFV expands rapidly by renal sodium retention and, in a number of conditions, also by dilution (osmoresetting). When sodium intake is zero, ECFV can only be achieved by water retention. As Baylis and Munger did not measure TBW, ECFV, serum vasopressin and glomerular filtration rate besides PV, their data do not exclude compensatory extra water retention as an explanation for PV expansion in early pregnancy.

From the present study we conclude that in normal rat pregnancy PV expansion is achieved by fluid retention rather than by a shift within the ECFV. 
The mechanism of volume adaptation in early rat pregnancy 


\section{Chapter 6}

\section{Hemodynamic changes in pseudopregnancy in chronically instrumented, conscious rats}

Brigitte F.M. Slangen ${ }^{1}$, Iris C.M. Out ${ }^{1}$, Carla M. Verkeste1, Jos F.M. Smits $^{2}$, Louis L.H. Peeters ${ }^{1}$

Departments of Obstetrics and Gynecology ${ }^{1}$ and Pharmacology ${ }^{2}$, University of Maastricht, 6200 MD Maastricht, The Netherlands.

American Journal of Physiology 272: H695-H700, 1997. 


\section{Abstract}

It is unclear whether the trophoblast is needed for the normal early-pregnancy hemodynamic adaptation. In this study we tested the hypothesis that the presence of trophobllast is not essential for the initial hemodynamic adaptation in pregnancy. To this end, we measured systemic hemodynamics in conscious pseudopregnant rats, and compared the results with those obtained in a concomitantly studied control group of virgin rats as well as with a previously studied group of pregnant rats. The rats were studied daily from day 4 postmating until day 10 and on days $12,14,18$ and 20. In pseudopregnant rats, cardiac output (CO) increased from day 5 onward, to $14 \pm 3 \%$ above the initial value by day 8 . This rise in $\mathrm{CO}$ was entirely accomplished by a rise in stroke volume ( $21 \pm 4 \%$ by day 8$)$. Mean arterial pressure did not change appreciably. Therefore, total peripheral resistance also decreased by $21 \pm 4 \%$ by day 8 . Meanwhile, peak flow, aortic flow acceleration and stroke work, indicators of myocardial performance, had increased, and the hematocrit had decreased ( $15 \pm 1 \%$ by day 8 ). Between day 10 and day 20 the hemodynamic parameters gradually returned to baseline. We conclude that systemic hemodynamic changes do take place in pseudopregnancy. They consist of a rise in $\mathrm{CO}$ by a rise in stroke volume, an increase in myocardial performance and hemodilution. The observed changes closely resemble those in early normal pregnancy. Therefore we accept our hypothesis that trophoblast is not essential for the initial hemodynamic changes in rat pregnancy. 


\section{Introduction}

The increase in cardiac output (CO) shortly after implantation seems to be a consistent phenomenon in mammalian pregnancy as suggested by observations in sheep (Rosenfeld 1977), rat (Slangen 1996), baboon (Phippard 1986) and human (Duvekot 1993, Robson 1989). CO increases without concomitant increase in nutritional flow demand, leading to a fall in systemic oxygen extraction (Gilson 1992) and a state of systemic hyperperfusion. The importance of this early increase in $\mathrm{CO}$ is indicated by the clinical experience that complications in advanced pregnancy, such as fetal growth restriction, are preceded by the absence or defective development of circulatory adaptations in early pregnancy (Duvekot 1995). It follows that the importance of this adaptive change in maternal hemodynamics is solely based on empiricism. Neither underlying mechanism nor physiological meaning of this phenomenon are understood.

In the rat, $\mathrm{CO}$ has increased on pregnancy day 8 , two days after implantation (Slangen 1996). At that time, luteal and trophoblastic hormones contribute to the prevailing endocrine environment (Garland 1987, Miller 1981). The importance of these two endocrine sources, as well as the contribution of immune-related factors triggered at the feto-maternal interface to the initial hemodynamic changes, are obscure.

Pseudopregnancy in the rat is characterized by cessation of the estrous cycle and persistence of the endocrine function of the corpus luteum. Pseudopregnancy in the rat lasts 12-14 days (Atherton 1982a, Garland 1987) and is in the first 9-10 days comparable to normal pregnancy with respect to circulating levels of steroids and prolactin (Garland 1987, Pepe 1974, Smith 1976). The two conditions only differ from each other by the presence or absence of the trophoblast. This biological difference enables us to study the contribution of the trophoblast to the hemodynamic changes in early pregnancy. The absence of these hemodynamic changes in pseudopregnancy would provide evidence for a crucial role of the trophoblast in this particular adaptation.

In this study, we tested the hypothesis that the presence of trophoblast is not essential for the initial hemodynamic adaptation in pregnancy. We measured systemic hemodynamics in conscious pseudopregnant rats, and compared the results with those obtained in a concomitantly studied control group of virgin rats as well as with a previously studied group of pregnant rats (Slangen 1996). 


\section{Methods}

ANIMAL PREPARATION Sixteen female Wistar rats (Winkelmann, Germany) were used, at the age of 3-4 months. All facilities and procedures were approved by the Ethical Board of the University of Limburg, Maastricht. At least one week of acclimatization to the centralized experimental animal facilities (12h:12h lightdark cycle) was allowed before surgery. Instrumentation was carried out aseptically under ketamine $(50 \mathrm{mg} / \mathrm{kg})$ and xylazine $(5 \mathrm{mg} / \mathrm{kg}$ ) anesthesia. Surgery was performed according to the method described previously (Slangen 1996, Smits 1982). Briefly, under positive pressure respiration a right thoracotomy was performed in the third intercostal space, and the ascending aorta was carefully dissected free from surrounding tissue. An electromagnetic flow probe $(2.3 \mathrm{~mm}$ Skalar, Delft, The Netherlands) was placed around the aorta at a distance of 3-4 $\mathrm{mm}$ from the heart. A silastic drain was inserted and subatmospheric intrathoracic pressure was restored by application of a negative pressure of $10 \mathrm{~cm} \mathrm{H} 2 \mathrm{O}$. The thorax was closed by pulling the ribs together with 3-0 silk. The probe cable was guided subcutaneously to the neck where the connector was secured to the skin. A polyethylene catheter $(0.28 \mathrm{~mm} \mathrm{ID,} 0.61 \mathrm{~mm} \mathrm{OD})$, filled with heparinized saline $(5 \mathrm{IU} / \mathrm{mll} 0.9 \% \mathrm{NaCl})$, was advanced into the abdominal aorta via the femoral artery. After guiding the catheter to the neck, it was plugged with a stainless steel pin.

EXPERIMENTAL PROTOCOL Rats destined to become pseudopregnant were mated with a vasectomized male $(n=8)$. Day one of pseudopregnancy was the day of presence of a vaginal plug. Successful induction of pseudopregnancy was confirmed by cessation of estrous cycling (persistence of the leucocytic vaginal smear in daily vaginal smears). Termination of pseudopregnancy is characterized by a return to proestrus, usually between day 12 and 14 after mating (Atherton 1982a, Garland 1987). In our study, return to proestrous occurred on day $13 \pm 1$ (mean \pm SD). Surgery, as described above, was performed on day 1 of pseudopregnancy. Each pseudopregnant rat was matched with respect to age, body weight and days postsurgery with a control rat $(n=8)$. Also, in the control group vaginal smears were performed. Estrous cycling continued after surgery. Weight loss after surgery was less than $5 \%$. Rats were studied daily from day 4 until day 10 and on days $12,14,18$ and 20 of pseudopregnancy or postsurgery. 
HEMODYNAMIC MEASUREMENTS Because of diurnal variations in hemodynamic variables (Montani 1995) all rats were studied at the same time of the day (between 10.00 and 15.00 ). All experiments were performed after calibration of the blood pressure transducer (Statham pressure transducer) and flow meters. Blood flow through the ascending aorta was measured using an electromagnetic flow meter (Skalar, Delft, The Netherlands). End diastolic flow through the aorta was taken to be zero on a beat-to-beat basis (Montani 1995). After connecting the arterial catheter and the flow probe to the measurement devices, data were recorded for 90-120 minutes. Mean values were stored on hard disk every 30 seconds after being analyzed by a real time data system. At the end of this period hematocrit (Hct) (vol\%; microcapillary method) was measured in duplicate $(100 \mathrm{ml}$ from the arterial catheter).

From the flow signal, cardiac output (minus coronary flow) $(\mathrm{CO} ; \mathrm{ml} / \mathrm{min})$, heart rate (HR; bpm), stroke volume (SV; $\mathrm{ml})$, peak aortic flow (PF; $\mathrm{ml} / \mathrm{min})$, and aortic flow acceleration ( $\mathrm{dF} / \mathrm{dt} ; \mathrm{ml} / \mathrm{s} 2)$ were derived. Systolic, diastolic and mean arterial pressure (MAP; $\mathrm{mmHg}$ ) were recorded from the arterial pressure signal. Due to catheter failure in two pseudopregnant and two control rats, MAP and Hct data could only be gathered from 6 rats in each group. Repeated sampling interfered with catheter patency. Therefore, the dropout rate of animals in which Hct and MAP data could be obtained increased progressively after day 8 . For this reason no statistical analysis was performed for these parameters on day 9 and after day 10 .

CALCULATIONS Calculated hemodynamic variables were total peripheral resistance (MAP/CO: TPR; mmHg.ml-1.min) and stroke work (MAP x SV: SW; mmHg.ml).

STATISTICAL ANALYSIS We used two-way analysis of variance by ranks (Friedman Test) to assess whether significant changes occurred within each group. If so, Willcoxon's signed rank test was subsequently employed to describe more precisely the changes within each group relative to day 4. To correct for the interindividual variation, data were expressed as \% change relative to day 4 postsurgery (Slangen 1996). Day 4 was selected as the standard day because on that day recovery from surgery was reached, as evidenced by the recovery of weight gain (Slangen 1996). Furthermore, the pseudopregnant and control groups were not statistically different on that day (Mann-Whitney U-test). Differences per day between the pseudopregnant and control groups were 
analyzed by Mann-Whitney U-test. Data are expressed as means $\pm S E$, unless stated otherwise. Differences were considered significant when the $\mathrm{p}$ was less than 0.05 .

\section{Results}

Mean body weight at surgery was $245 \pm 13 \mathrm{~g}$ and $240 \pm 11 \mathrm{~g}$ (means $\pm \mathrm{SD}$ ) in the pseudopregnant and control group, respectively. In two pseudopregnant rats the aorta ruptured (on day 11 and day 19 postsurgery, respectively). Before that event, these rats appeared healthy, as also reflected in normal hemodynamic values. At autopsy there were neither signs of infection nor of fibrosis around the ascending aorta. Therefore, the data of these rats were included in the later analysis. One pseudopregnant rat could not be measured after day 14 due to technical problems with the flow probe.

Hemodynamic values in 4-day pseudopregnant animals were similar to those of controls (table 1). In pseudopregnant rats, $\mathrm{CO}$ increased from day 5 onward, to reach a value of $14 \pm 3 \%$ above the initial value by day $8(\mathrm{p}<0.05$; figure 1$)$. This rise in CO was paralleled by a rise in SV $(21 \pm 4 \%$ by day $8 ; \mathrm{p}<0.05)$ and a $21 \pm 4 \%$ fall in TPR by day 8 ( $p<0.05$; table 1$)$.

Figure 2 depicts the percentage changes with pseudopregnancy or postsurgery, relative to the 4th day, in $\mathrm{PF}, \mathrm{dF} / \mathrm{dt}$ and $\mathrm{SW}$, indicators of myocardial performance (Slangen 1996). PF and $\mathrm{dF} / \mathrm{dt}$ had increased in the pseudopregnant group, relative to the control group, on day 5 and between days 8 and 14 postmating. In pseudopregnant rats, SW had increased, relative to day 4, between days 6 and 10 . These hemodynamic changes were accompanied by a $15 \pm 1 \%$ fall in $\mathrm{Hct}$ by day 8 of pseudopregnancy (figure 1). Also, in the control group a fall in Hot was observed. However, this fall was smaller than that in the pseudopregnant group between days 8 and $10(p<0.01$; figure 1$)$.

In the pseudopregnant group all variables gradually returned to baseline after day 10 , to reach values comparable to control rats by day 20 postmating (figures 1 and 2, table 1).

HR decreased in both groups from day 5 (pseudopregnant group), and day 12 (control group) onward. A fall in MAP was observed in the NP group only (days $6,8$ and 10 ; table 1$)$. 
Twble 1 Cardionascular variables in pseudopregnancy

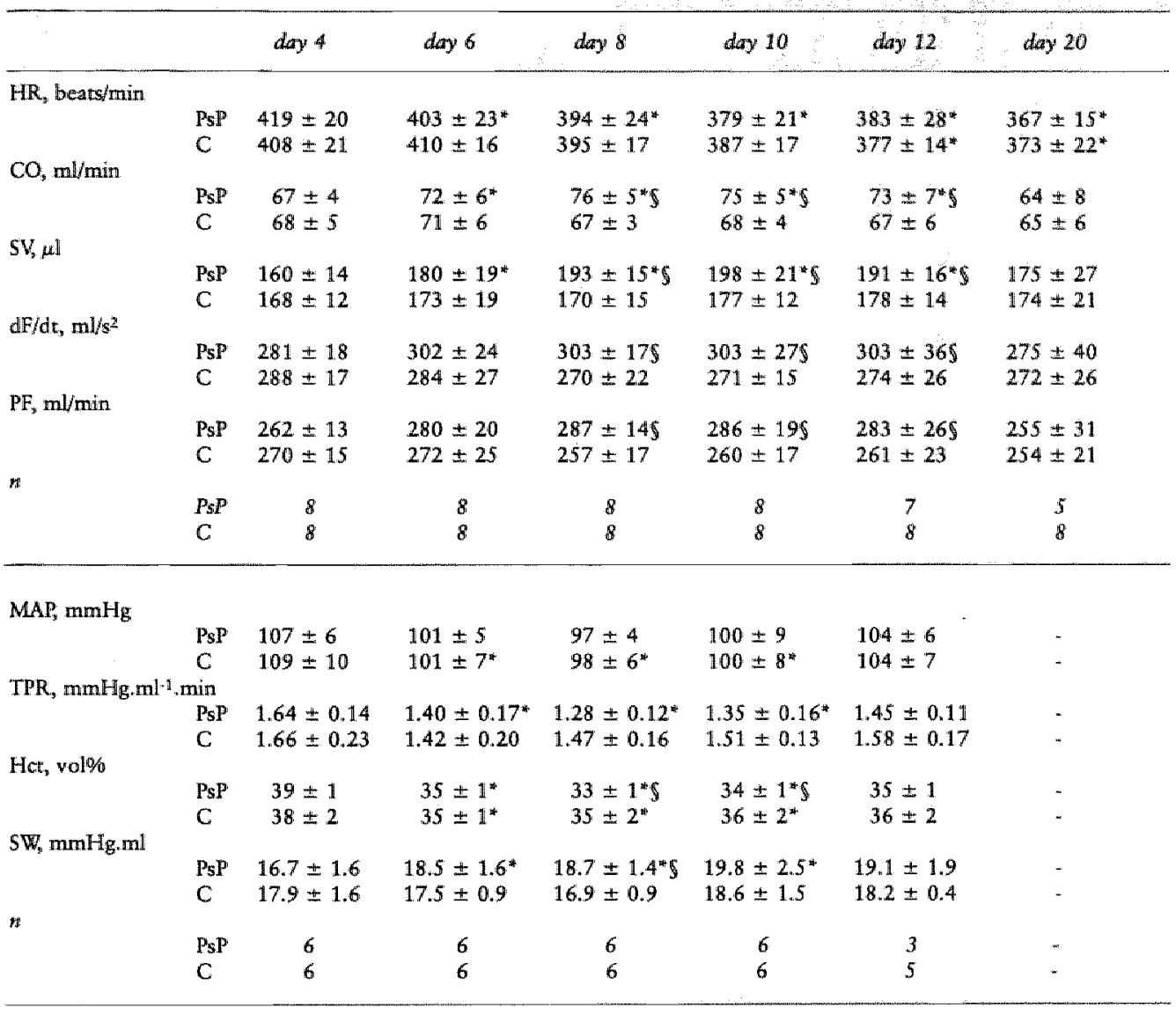

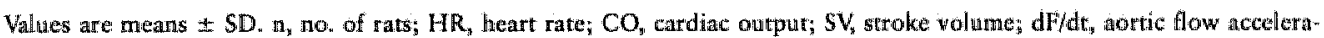

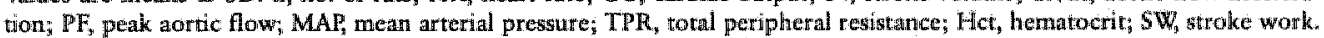
Each variable was subjected to 2*way analysis of wariance by ranks (Friedman test). Pseudopregnatut group (Psp): $\mathbb{P}^{p}<0.01$

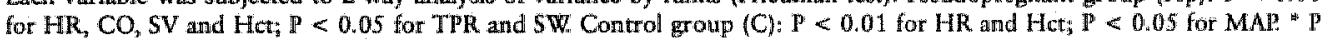
$<0.05$ compared with day 4 by Wilcoxon's signed rank tests, $\$ P<0.05$ compared witl control rats by Mannwhititey $U$. test. 


\section{Chapter 6}

\section{Figure 1}

Percentage changes, relative to day 4 , in cardiac output (CO), stroke volume (SV) and hematocrit (Hct) in pseudopregrnant ( $\nabla$ ) and control rats ( $($ ). Values are means \pm SE. $p<0.05$ compared with day 4 by Whlcowon"s signed rank tegt, $\mathrm{p}<0.05$ poudopregnanit rats compared with control rats by Mann-Whitney Untest.
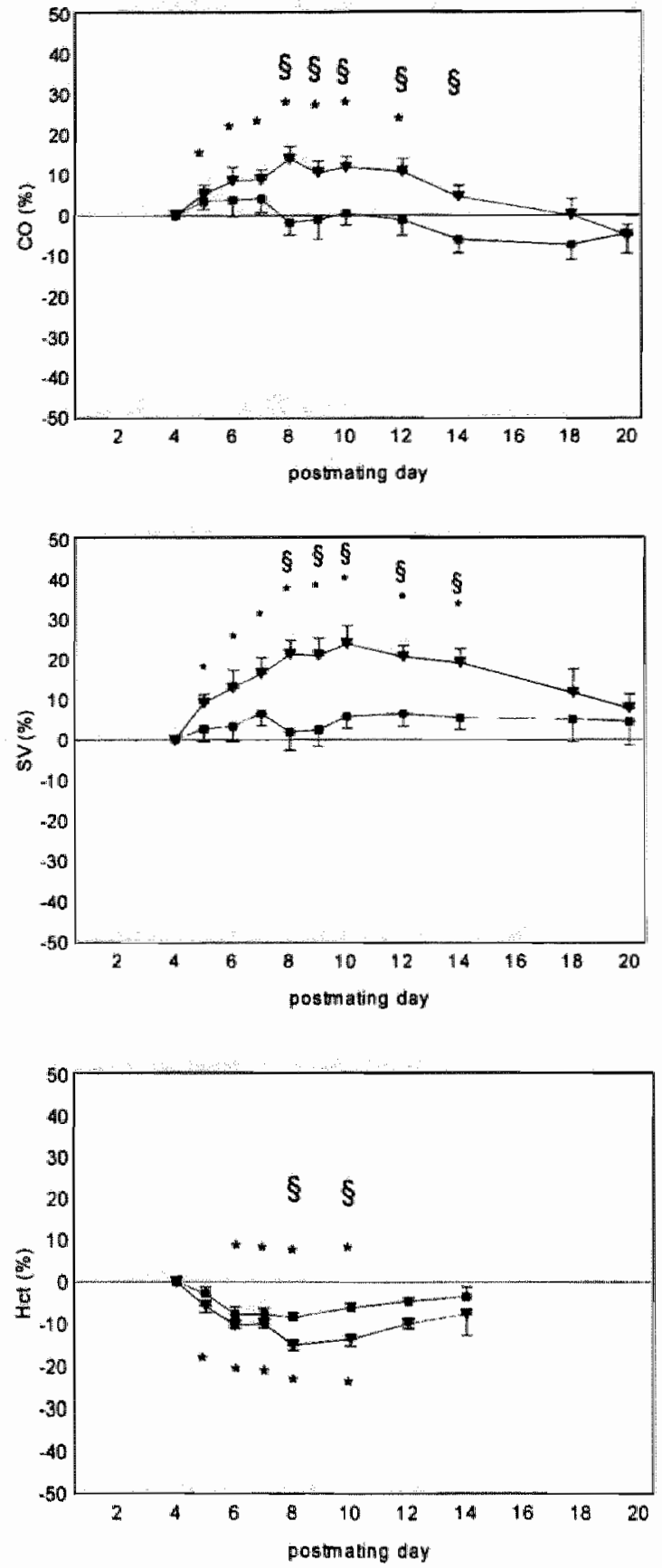


\section{Fughe 2}

Percentage changes, relative to day 4, in peak aortic tlow (PF), aortic flow acceleration (dF/dr) and stroke work (SW) in psendopregnant ( $\boldsymbol{\nabla}$ ) and control rats (w). Values are means $\pm S E{ }^{*} p<0.05$ compared with day 4 by Wilcoxon's signed rank test, $\$ \mathrm{p}<0.05$ pseudopregnant rats compared with control ras by Mann.Whitney U-rest.
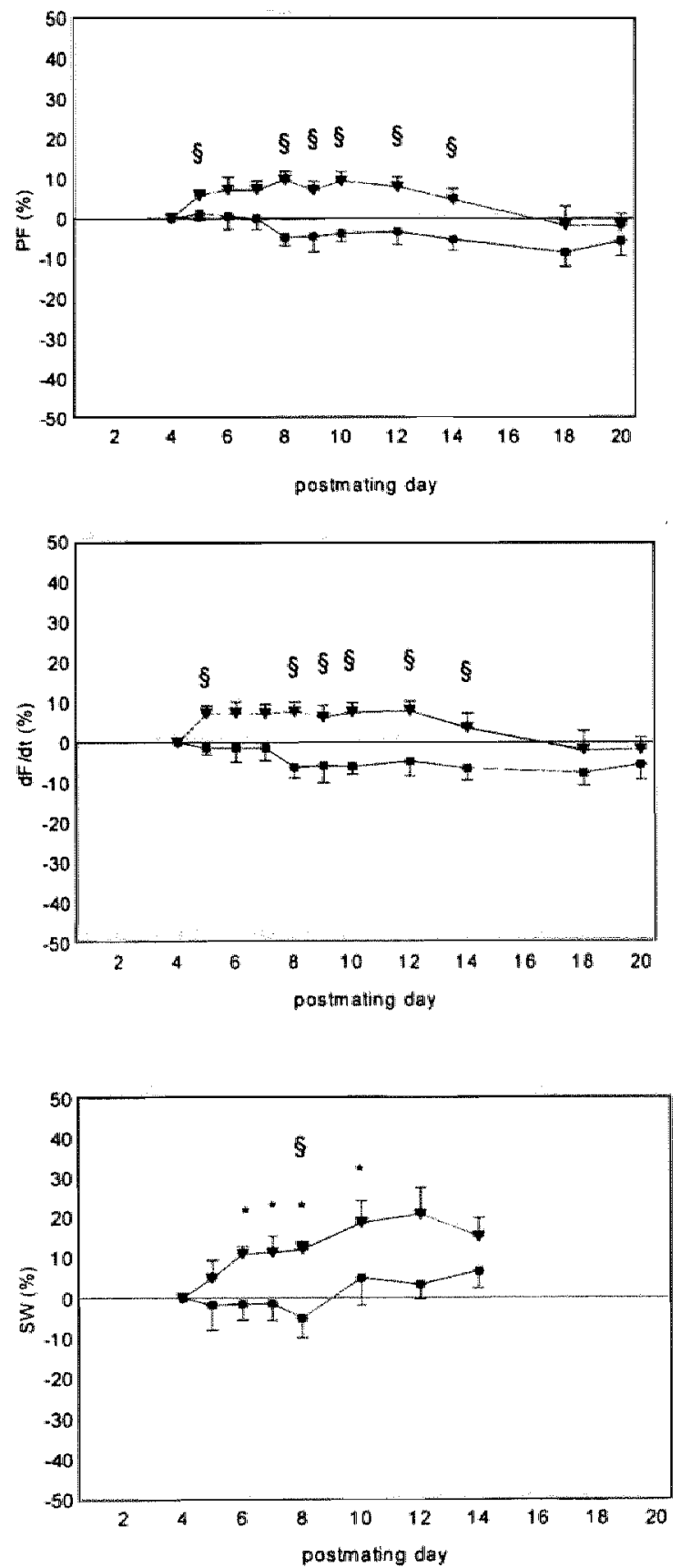


\section{Discussion}

Pseudopregnancy in the rat is a state in which hormonal, volume, and renal changes develop that resemble closely those in early normal rat gestation (Baylis 1982, Smith 1975, Smith 1976). Hot decreases, and plasma volume and extracellular volume increase (Atherton 1982a, Baylis 1982). Glomerular filtration rate (GFR) increases between days 7 and 12 postmating, gradually returning to values comparable to virgin rats in the subsequent 3-4 days (Atherton 1982a). To our knowledge, no studies have been performed to study the systemic hemodynamic changes in pseudopregnancy. Previous studies in the first half of rat pregnancy failed to demonstrate changes in maternal hemodynamics, most likely in conjunction with the relatively large measurement error associated with the techniques employed (Ahokas 1983, Dowell 1993, Gilson 1992). Obviously, the hemodynamic changes in pseudopregnancy would be even more difficult to identify (Pang 1979). Recently, we used chronically implanted electromagnetic flow probes, to measure central hemodynamics in conscious, unrestrained, pregnant rats. In this model, we found that $\mathrm{CO}$ had already increased significantly by day 8 of rat pregnancy (Slangen 1996). The present study shows similar systemic hemodynamic changes in pseudopregnant rats, using the same experimental design as in our previous study in pregnant rats (Slangen 1996). The changes in $\mathrm{CO}$ in both studies, are shown in figure 3. At first sight, the changes in $\mathrm{CO}$ in pregnancy and pseudopregnancy follow a similar pattern until day 9 . In both conditions, the CO has increased consistently and by comparable fractions by day 8 . However, after this day the patterns begin to deviate: after one day of little change, the $\mathrm{CO}$ rise resumes in the pregnant rats. In contrast, the elevated $\mathrm{CO}$ in the pseudopregnant rats gradually returns to the nonpregnant level over a period of 10 to 12 days. This similarity up to day 9 not only applies to $\mathrm{CO}$, but also to the other central hemodynamic parameters, thus suggesting a similar mechanism responsible for the initial hemodynamic changes in pregnancy and pseudopregnancy. In both studies, the increase in $\mathrm{CO}$ is accomplished by a selective rise in SV, and is accompanied by a fall in TPR and a rise in myocardial performance. The fall in HR and MAP (only in the control group) in the present study was also observed in our previous study (Slangen 1996), and seems to reflect habituation to the experimental procedure. In the present study, we observed a fall in Hct between days 4 and 8 in both the experimental and control group. In our previous study (Slangen 1996) the Hct did not change in the control group. The rea- 


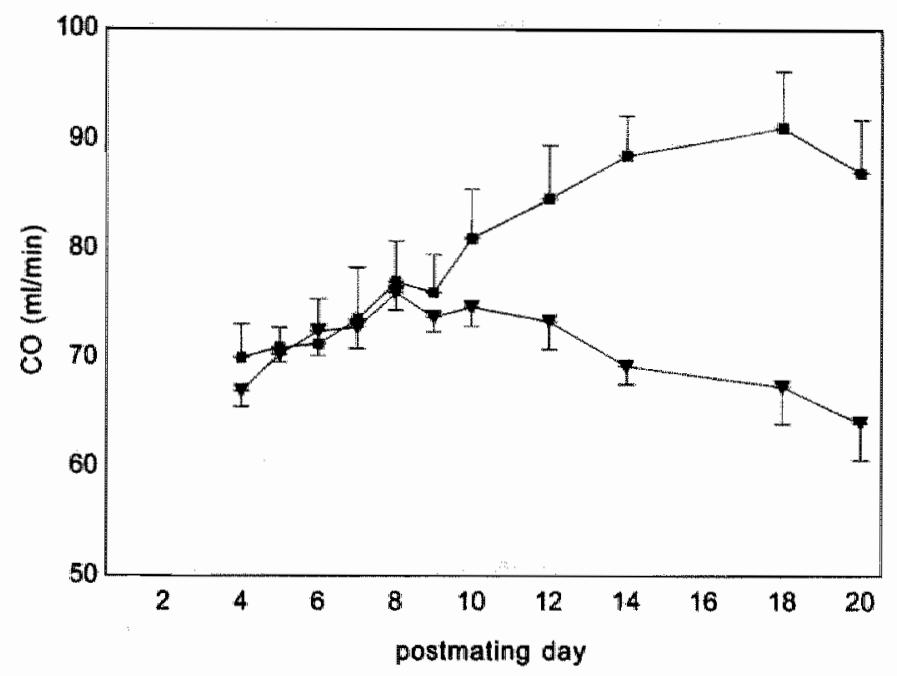

son why the Hct has decreased in the present control group is obscure, since this group was managed identically as our previous control group. Nevertheless, the larger fall in Hct by day 8 in pseudopregnant rats relative to that in the control group, may be secondary to plasma volume expansion, as has also been reported by others in pseudopregnancy (Baylis 1982).

It has been suggested that plasma volume expansion in this condition develops in response to arterial vasodilation, since the latter triggers a non-osmotic ADHrelease (Schrier 1991). The vasodilation, in turn, is thought to develop in response to a rise in nitric oxide (NO) release by the vessel wall. This is suggested by the higher urinary excretion of nitrate and cGMP both in earlly pregnancy and in pseudopregnancy in the rat (Conrad 1993). However, these observations do not provide information on the mechanism responsible for the increased NO production in early pregnancy. Our results in the pseudopregnant rat indicate that the early trophoblast may not be involved in initiating the early-pregnancy rise in NO synthase activity. Apparently, neither trophoblastic hormones nor immunerelated effects of the trophoblast play a role in the initial development of the cardiovascular changes in pregnancy. 
During the first 8 days of rat pseudopregnancy the peripheral levels of progesterone, $17 \mathrm{~B}$-estradiol and prolactin resemble closely those in normal rat pregnancy, with progesterone gradually increasing within 48 hours after mating (Atherton 1982a, De Greef 1977, Garland 1987, Miller 1980, Smith 1976), prolactin developing a pattern that consists of 2 daily surges within hours after mating (Smith 1975, Smith 1976) and circulating levels of 17 B-estradiol remaining low and nonrising (Garland 1987). The similarity in endocrine profile in early rat pregnancy and pseudopregnancy supports the view that at least one of these 3 hormones is involved in triggering the hemodynamic adaptation. The low and nonrising level of 17 B-estradiol during the first 8 days of (pseudo)pregnancy makes this steroid an unlikely candidate to be responsible for the initial hemodynamic changes, although $17 \mathrm{~B}$-estradiol is known to have vasodilating properties resembling those in pregnancy (Magness 1993, Rosenfeld 1976, Weiner 1994b). Since also the vaso-active properties of both progesterone (Hart 1985, Nakamura 1988) and prolactin (Bryant 1973, Kaufman 1981, Mills 1981) in physiologic concentrations are modest, it seems highly unlikely that the induction of hemodynamic adaptation in early pregnancy can be explained by some simple doseresponse relationship between the three luteal hormones and the cardiovascular system. Based on the hemodynamic data from the present - and our previous study (Slangen 1996), and the well-described endocrine data in early pregnant and pseudopregnant rat (De Greef 1977, Garland 1987, Miller 1980, Pang 1979, Pepe 1974, Smith 1975, Smith 1976), we speculate that one of the three luteal hormones, or a combination, triggers the development of peripheral arterial vasodilation only after they have circulated for at least 6 days. It is possible that the institution of a high cardiac output-low resistance circulation is a direct or indirect endocrine effect. However, it is also possible that the high flow-low resistance circulation develops in response to a still unknown potent vasodilating factor released by the ovaries or endometrium after a certain exposure to the prevailing endocrine environment. In that case, this factor could act either directly on the vessel wall or indirectly by increasing the affinity of $17 \mathrm{~B}$-estradiol to its receptor in the vessel wall. Since $17 \mathrm{~B}$-estradiol induces the expression of endothelial NO synthase (Weiner 1994b), 17 B-estradiol may contribute to the increased NO production and to the vasodilated state of pregnancy. This indirect mechanism may explain the absence of any profound increase in 17 B-estradiol levels at the time of maximum hemodynamic changes. The vasodilating effect 
Hemodynamic changes in pseudopregnancy in chronically instrumented, conscious rats

gives rise to an afterload reduction and a state of arterial underfill, with secondary expansion of the plasma volume (Schrier 1991).

We conclude that, until day 9 postmating, pseudopregnancy is characterized by similar systemic hemodynamic changes as observed in pregnancy. These changes include peripheral vasodilation, a rise in $\mathrm{CO}$ by a selective rise in $\mathrm{SV}$, and an increase in myocardial performance. This observation implies that, at least in the rat, the trophoblast is not essential. for the initial maternal hemodynamic adaptation to early pregnancy. 


\section{Chapter 7}

The short-term effect of estradiol-I $7^{\beta}$ on skeletal and renal blood flow in awake, ovariectomized rats

Brigitte F.M. Slangen, MD, Iris C.M. Out, Carla M. Verkeste, $\mathrm{MD} \mathrm{PhD}$, Louis L.H. Peeters, MD PhD.

Department of Obstetrics and Gynecology, University of Maastricht, 6200 MD Maastricht, The Netherlands.

Submitted. 


\section{Abstract}

The pregnant and pseudopregnant rat have in common that they develop a highflow low-resistance circulation in the first 9 days postmating, most likely secondary to generalized vascular relaxation. This suggests that the throphoblast is not involved in the initial pregnancy-related vasorelaxation. Estradiol-17B (E2) is an ovarian steroid, that is present in the peripheral blood both in pregnancy and pseudopregnancy with known vasodilating properties. This study was designed to explore the immediate macrocirculatory response to $\mathrm{E} 2$ to determine whether the initial acceleration in the luteal release of this steroid alone can be kept responsible for the induction of the vasorellaxation.

Rats were ovariectomized and fitted with Doppler flow probes around vessels supplying distinctly different tissues, the abdominal aorta (predominantly skeletal tissue and skin) and a renal artery. A catheter was inserted into the abdominal aorta to measure mean arterial pressure $(\mathrm{mmHg})$. After adequate recovery the rats received either a subcutaneous injection of $10 \mathrm{mg} \mathrm{E} 2(n 40 \mathrm{mg} / \mathrm{kg} ; \mathrm{n}=8)$ or its vehicle (arachis oil; $n=5$ ). Hindquarter blood flow, renal blood flow and mean arterial pressure were recorded continuously from 1.5 hours before, until 4 hours after the $\mathrm{E} 2$ or vehicle administration.

E2 induced a gradual increase in hindquarter blood flow to a maximum of $20 \pm$ $6 \%$ (SEM) above baseline, at 2.5 hours after injection. Mean arterial pressure decreased gradually to a nadir of $9 \pm 2 \%$ below baseline at 3.5 hours after E2 injection. Neither heart rate nor renal blood flow changed appreciably in response to E2. Vehicle injection did not induce any consistent change in the variables.

Although $\mathbb{E} 2$ induced a gradual decline in mean arterial pressure, resembling that in pregnancy, its effect on blood flow distribution was different. In contrast to the concomitant rise in renal blood flow and skeletal blood flow in early pregnancy, E2 induced a selective rise in hindquarter blood flow only. Therefore, we conclude that E2 alone and given as a bolus, is not the trigger for the initial pregnancy-related vascular relaxation. However, it is possible that the initial pregnancy-related vasorelaxation develops gradually after sustained E2 exposure, probably in concert with some other vasoactive (luteal or decidual) factor. 


\section{Introduction}

Many hemodynamic and volume changes in early mammalian pregnancy seem to develop in response to a generalized fall in vascular tone (Duvekot 1993, Phippard 1986, Slangen 1996). The resulting high-flow low-resistance circulation is accomplished by a combination of distal (arteriolar) vasodilation and proximal vasorelaxation (increased aortic compliance) (Hibbard 1994, Slangen 1996, Slangen 1997). The trigger for this phenomenon is not known. Previously, we observed in the pregnant and pseudopregnant rat that the onset of hemodynamic and volume changes (hemodilution) was already noticeable by day 6 postmating (day of implantation), to become significant by day 8 (Slangen 1996, Slangen 1997a). The resemblance between pregnancy and pseudopregnancy in this respect, as also reported by others (Baylis 1982, Conrad 1989), suggests that the trophoblast may not be involved in the initial adaptations to pregnancy. Consequently, a hormone of ovarian origin - alone or in concert with some other e.g. decidual factor - triggers the development of the high-flow low-resistance circulation. Estradiol-17ß (E2) is a potential candidate because of its vasodilating effects (Davis 1989, Farhat 1996, Hart 1985, Magness 1989, Slater 1986). Previous studies in sheep indicate that the circulation exposed to acute E2 stimulation responds by a rise in stroke volume and cardiac output without a concomitant change in MAP (Magness 1989, Rosenfeld 1976, Ueland 1966), adaptations resembling those in early pregnancy (Duvekot 1993, Slangen 1996). The contribution of E2 to the gestation-induced renal vasodilation is unclear, and has, to the best of our knowledge, only been studied in sheep. In the sheep, E2 does not induce renal vasodilation (Rosenfeld 1976), however, it seems that renal blood flow does not increase in pregnancy in this species (Rosenfeld 1977). In the immediate postconceptional period, E2 levels are low, but increase gradually with advancing pregnancy (Challis 1971, Garland 1987, Mishell 1973, Rosenfeld 1976). In early pregnancy and pseudopregnancy in the rat, initial E2 levels remain low (Bridges 1984, Gallo 1985, Garland 1987, Smith 1975) except for an E2 surge on the afternoon of day 4 postmating (Shelesnyak 1963, Yoshinaga 1969).

In the present study, we tested the hypothesis that the earliest rise in circulating E2 levels in rat pregnancy triggers the initial nonselective vasorelaxation of pregnancy, as will be reflected in higher blood flows to both the kidney and skeletal tissue. To this end, we studied hindquarter blood flow and renal blood flow in 
awake, chronically instrumented rats before and after a bolus injection with E2. To avoid endogenous fluctuations in E2 levels, rats were ovariectomized.

\section{Materials and methods}

ANIMAL PREPARATION Ten female virgin Wistar rats (Winkelmann, Germany) were studied, at the age of 3-4 months. All procedures were approved by the Institutional Animal Care and Use Committee of the University of Limburg, Maastricht. Rats were kept for at least one week on a 12-hours light/12-hours dark cycle, with water and food ad libitum, in the centralized experimental animal facilities. Surgery was carried out aseptically under ketamine $(40 \mathrm{mg} / \mathrm{kg})$ and xylazine $(4 \mathrm{mg} / \mathrm{kg}$ ) anesthesia. Following a mid-abdominal incision, the rats were ovariectomized and $20-\mathrm{MHz}$ directional pulsed Doppler flow probes (ValpeyFisher Corporation, Hopkinton MA, USA) were placed on the right renal artery (internal diameter $0.7 \mathrm{~mm}$ ) and the abdominal aorta (internal diameter $1.2 \mathrm{~mm}$ ), distal to the renal arteries. The abdomen was closed and the probe cables were guided subcutaneously to the neck where the cables were stored in a small Eppendorf cup. A poly-ethylene catheter $(0.28 \mathrm{~mm}$ ID; $0.61 \mathrm{~mm}$ OD; Portex, Medica BV, Den Bosch, The Netherlands) filled with heparinized saline $(5 \mathrm{IU} / \mathrm{ml}$ $0.9 \% \mathrm{NaCl}$ ), was advanced into the abdominal aorta via the left femoral artery. The catheter tip was positioned below the abdominal doppler probe. The catheter was then tunneled subcutaneously, exteriorized between the scapulae and plugged with a stainless steel pin. A single subcutaneous injection of $5 \mathrm{ml}$ saline was administered to compensate for loss of fluid during surgery.

EXPERMENTAL PROTOCOL Animals were allowed to recover for at least 6 days after surgery. Because of diurnal variations in hemodynamics (Smith 1987) all rats were studied at the same time of the day (between 10.00 and 15.00). After connecting the arterial catheter and the flow probe to the measurement devices, data were recorded for 90-120 minutes. Then, a subcutaneous injection of $0.25 \mathrm{ml} \mathrm{E2}$ (40 $\mathrm{mg} / \mathrm{ml}$, Org 2317, N.V. Organon, Oss, The Netherlands; $\mathrm{n}=8$ ) or vehicle (arachis oil; $\mathrm{n}=5$ ) was administered. Three rats received both E2 and vehicle, one received E2 first and ten days later vehicle, 2 rats received vehicle first followed by $\mathrm{E} 2$ one and two days later, respectively. These injections lead to maximum 
plasma levels 5 -fold above the physiological levels, with a 3 -fold increase being reached after 1 hour (personal communications, F. Dekkers, N.V. Organon, Oss; The Netherlands).

HEMODYNAMIC MEASUREMENTS All experiments were performed after calibration of the blood pressure transducer (Statham pressure transducer) and flow meters. An estimate for blood flow, but more importantly blood flow changes, was obtained using a 20-MHz directional pulsed Doppler flowmeter (Bio-engineering Resource Facility of Iowa, Iowa City, Ia, USA). Changes in regional blood flow were measured as changes in $\mathrm{kHz}$ Doppler shift, which are directly and linearly proportional to volume flow (Haywood 1981). Zero blood flow was determined electronically. During each experiment continuous data were obtained for heart rate (HR), mean arterial pressure (MAP), hindquarter blood flow (HBF) and renal blood flow (RBF). Pulsatile flow and pressure signals were recorded beat to beat on tape. Mean values were stored on hard disk every $30 \mathrm{sec}$ after having been analyzed by a real time data system.

CALCULATYONS Baseline values were determined by averaging measurements during the last 10 minutes of the baseline period, when the rat was resting quietly. After injection, \% change relative to baseline was calculated for consecutive 30 minutes intervals, by averaging 8 measurements at 30-s intervals.

STATISTICAL ANALYSIS Data are expressed as means \pm SE. E2 and vehicle effects were tested relative to baseline (Wilcoxon's signed rank test). Values for HR, MAP and body weight in baseline in the E2 and control group were compared by Mann-Whitney U-test. Differences were considered significant when $\mathrm{P}$ was less than 0.05 .

\section{Results}

Baseline values for HR, MAP, body weights and days of recovery after surgery did not differ between the groups (table 1). Figures 1 to 6 show the percentage changes in MAP, HR, hindquarter blood flow (HBF), hindquarter resistance (HQR), renal blood flow (RBF), and renal resistance (RER), respectively, after E2 
or vehicle injection. From two hours after injection of E2 onward, MAP decreased gradually to a nadir of $-9 \pm 2 \%$ which was reached 3.5 hours after injection (fig 1). HR did not change appreciably, except for a slight, but significant increase 1.5 hours after injection (fig 2). After E2 administration, HBF increased gradually, to a maximum of $20 \pm 6 \%$ at 2.5 hours after injection. HQR fell by $21 \pm 4 \%$ (figures 3 and 4). RBF and RER remained unchanged (figures 5 and 6). In the control group, none of the variables measured changed significantly in response to vehicle (figures 1-6).

Table1 Baseline values in estrogen-treated and control rats

\begin{tabular}{lcc} 
& E2 & Control \\
\hline MR, beats/min & $346 \pm 33$ & $337 \pm 14$ \\
MAP, ming & $104 \pm 11$ & $108 \pm 8$ \\
BW, surgeryg & $276 \pm 44$ & $299 \pm 51$ \\
BW, experiment, g & $279 \pm 42$ & $296 \pm 44$ \\
Recovery period, days & $9 \pm 5$ & $10 \pm 5$ \\
m & & 5
\end{tabular}

Values are means \pm SD. HR, heart rate; MAP, mean arterial pressure; BW, body weight, Recowery period, number of days between surgery and experiment; $n$, number of rats. 
Figute: 1

Percent changes, relative to baseline, in mean arterial pressure (MAP) after injection of estradiol 17 (1) (upper panel; $\mathrm{m}=8$ ) or arachis oil (lower panel; $n=5$ ). Values are means $\pm \mathrm{SD}, * \mathrm{P}<0.05$ compared with baseline by Wilcoxon:s signed rank test.
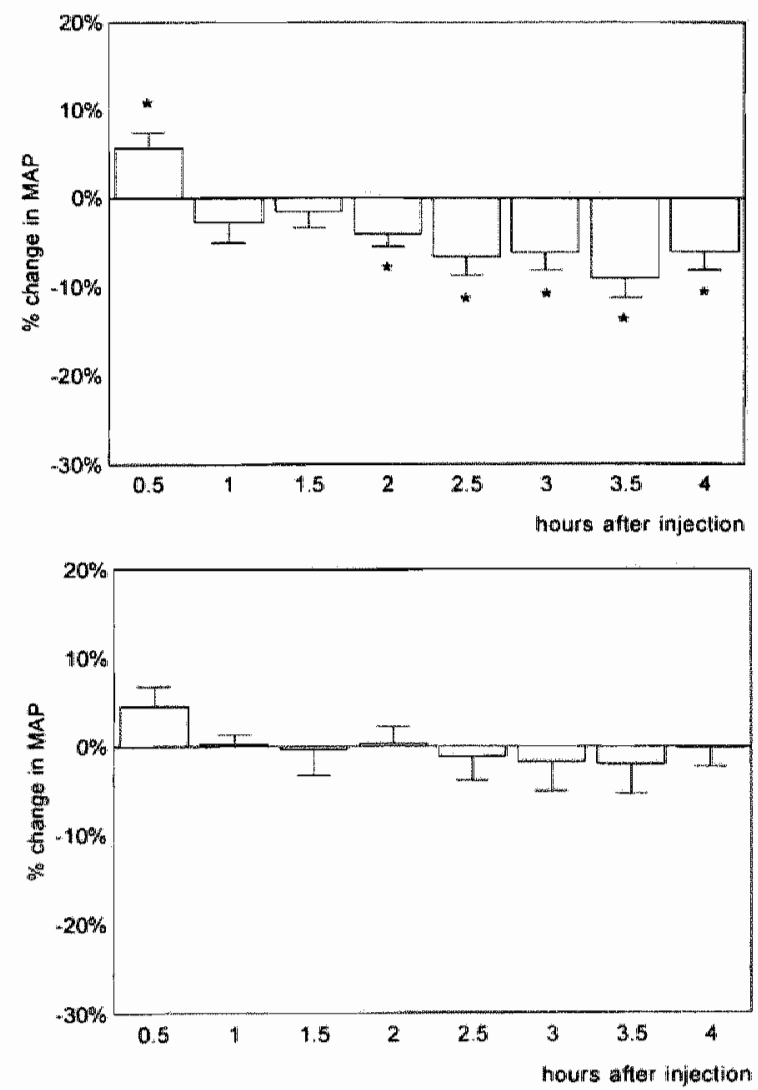
Chapter 7

Fitgure 2

Percent changes, relative to baseline, in heart rate (HR) after injection of estradiol-17Q (upper panel; $n=8$ ) or arachis oill (lower painel; $\mathrm{n}=5 \mathrm{f}$ ). Vallues are means $\pm \mathrm{SD} * \mathrm{P}<0.05$ compared with baseline by Wilcoxon's signed rarke test.
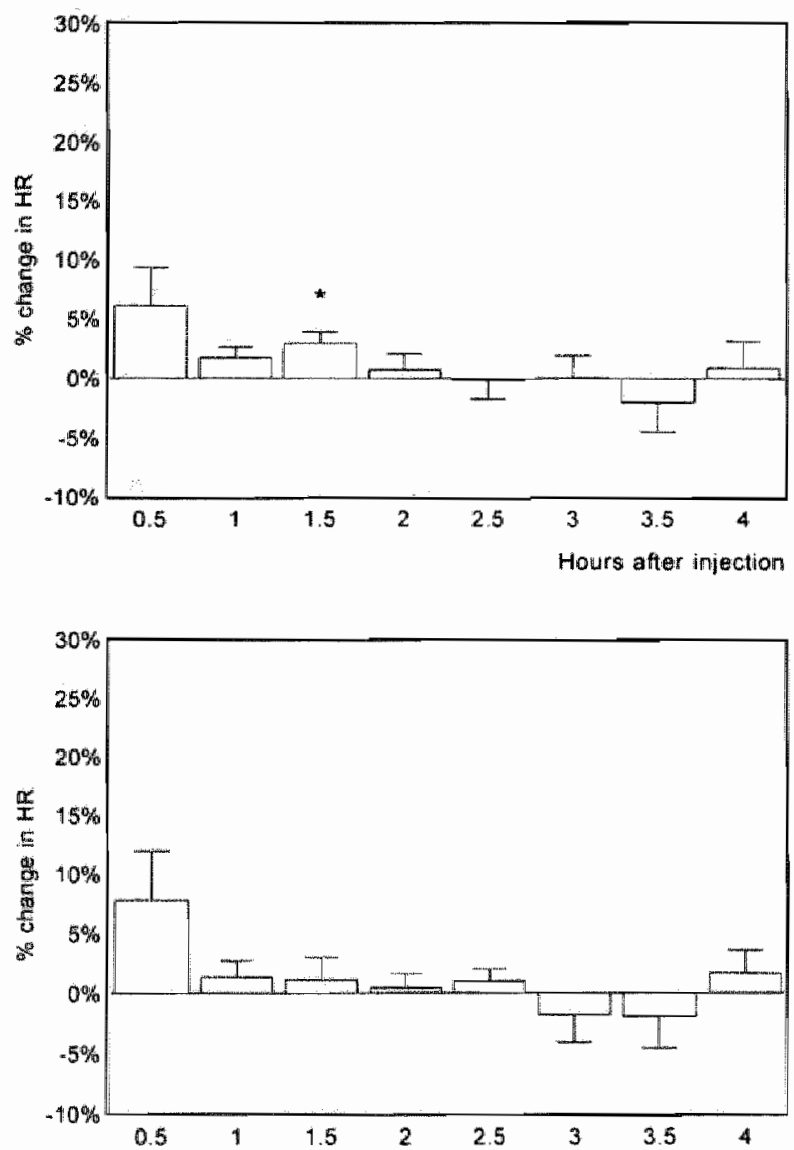
Figure 3

Percent changes, relative to baseline, in hindquarter blood How (HBF) after injection of estradiol-17B (upper panel; $\mathrm{n}=8$ ) or arachis oil (lower panel; $\mathrm{n}=5$ ). Values are means \pm SD $* \mathrm{P}<0.05$ compared with baseline by wilcoxon's signed rank test.
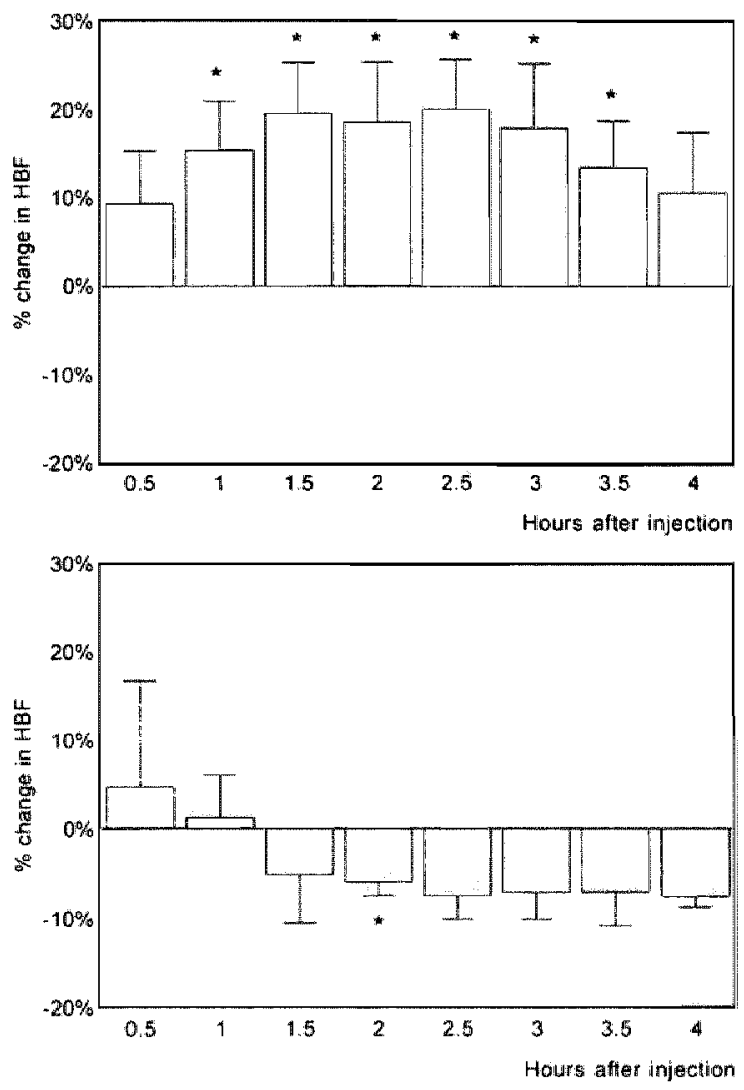


\section{Chapter 7}

Fisure 4

Percent changes, relative to basieline, in hindquarter resistance (HQR) after injection of estradiol-178 (upper panel; $n=8$ ) or arachio oil (lower pand; $n=5$ ). Values are means $\pm 5 D * P<0.05$ compared with baseline by Wilcoxon's isgued rank testit
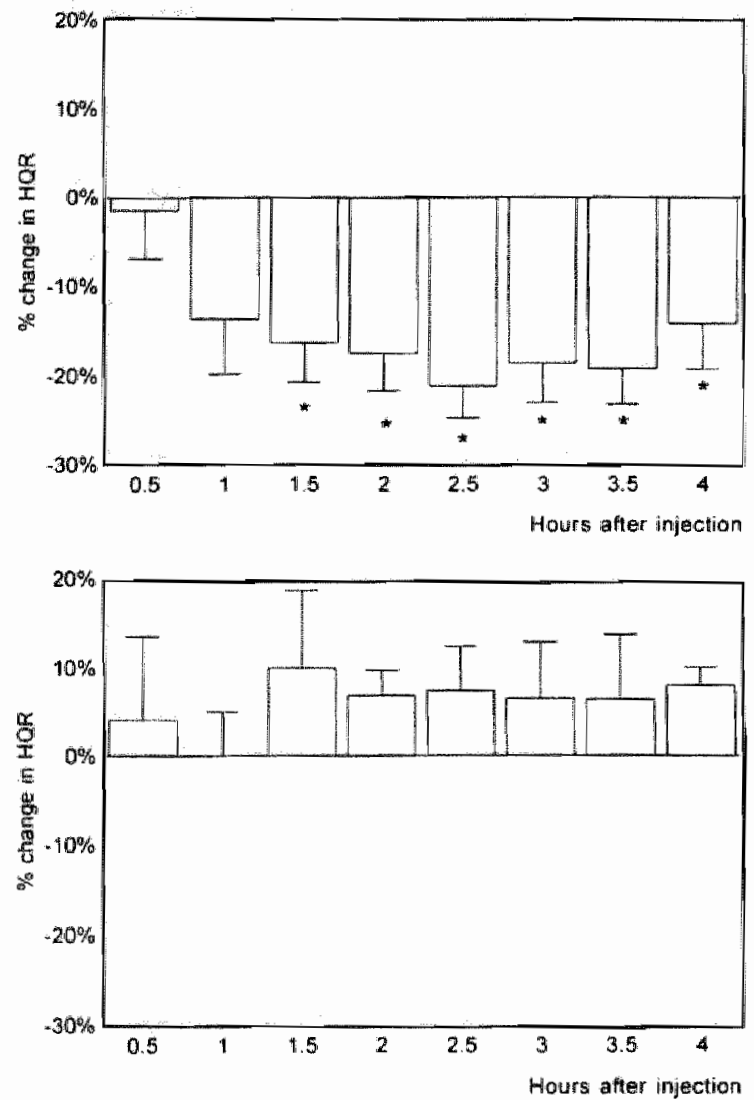
Figure 5

Percent changes, relative to baseline, in renal blood flow (RBF) after injection of estradiol-17B (upper panel; $n=8$ ) or arachis oil (lower panel; $n=5$ ). Values are means $\pm 5 D$. $P<0.05$ compared with baseline by Wilcoxom "sigmed rank test.
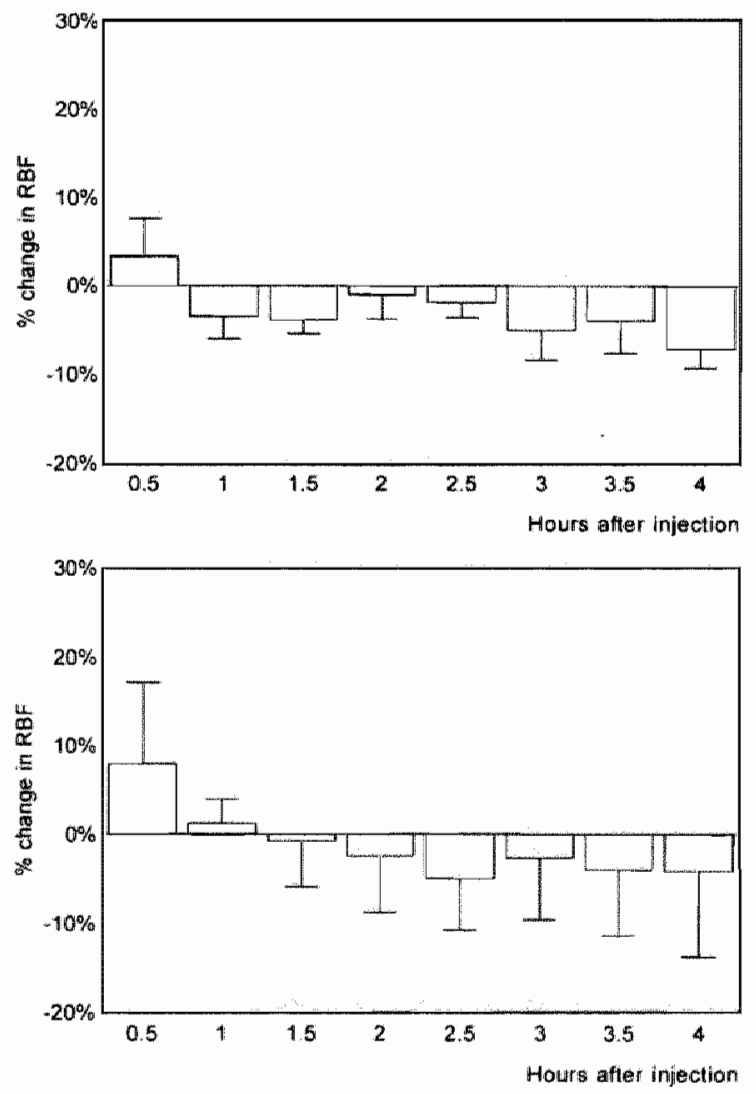


\section{Chapter 7}

\section{Figure 6}

Percent changes, rellative to baselinte, in renal resistance (RER) after injection of estradiol-17\& (upper panel; $n=8$ ) or arachis oil (lower panel; $\mathrm{n}=5$ ). Values are means $\pm \mathrm{SD} . \mathrm{P}<0.05$ compared with baselline by Whicoxom's sigmed ranllk tresit.
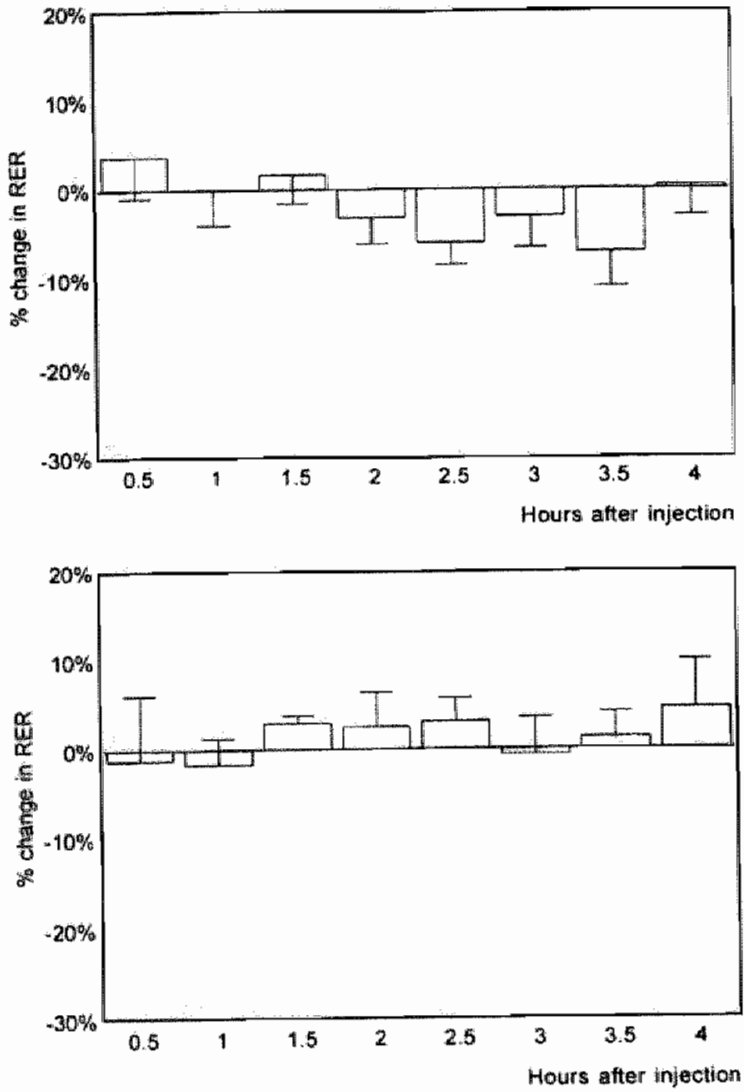


\section{Discussion}

In this study we determined in ovariectomized rats, the shortterm effects on the central circulation of E2, a steroid with well-known vasodilating properties (Farhat 1996). The fact that E2 receptors have been found in the heart, aorta ( $\mathrm{Lin}$ 1985, McGill 1981), and in the renal artery (McGill 1981) indicates that the cardiovasular system is a target for this steroid. Previous studies have shown that acute E2 infusion in sheep induces a rise in cardiac output and stroke volume, a fall in total peripheral resistance, and no change in MAP. The response of HR was variable, some authors reporting a rise (Magness 1989, Rosenfeld 1976) and others no change in HR (Ueland 1966). Prolonged treatment with E2 induces a consistent rise in cardiac output and stroke volume and a trend to a lower MAP in guinea pig (Hart 1985), sheep (Magness 1993), and human (Slater 1986). The blood flows to skin and myocardium are also increased (Rosenfeld 1976). However, few studies have investigated the specificity of E2-induced vasodilation. Our finding of an E2-induced vasodilation in the vasculature supplying skeletal muscle and skin, agrees with the observations in sheep (Rosenfeld 1976). Our study also demonstrates that E2 does not induce vasodilation in the renal artery, which is similar to observations in sheep (Rosenfeld 1976). It seems, however, that this species does not have an increase in renal flow in pregnancy (Rosenfeld 1976). Furthermore, the lack of a renal response in the latter studies may be related to the technique employed, as the microsphere technique using a single injection is associated with a measurement error of 15-20\% (Buckberg 1973). Also, microspheres were injected at the time of maximal response of the uterine artery to E2 (Rosenfeld 1976), which does not necessarily respond to the time of maximal response of the kidney or skeletal muscle. Indeed, when labeled E2 is administered to rats, the level of radioactivity in kidney, liver and muscle reaches a maximum within 15 minutes. In contrast, incorporation of the labeled hormone by the uterus follows a different pattern, with radioactivity peaking at 60 to 90 minutes, while the blood concentration is falling again (Christy 1974). Other studies have been performed in intact animals so that the possible modulating influences of endogenous E2 and progesterone on hemodynamics cannot be excluded (see Christy 1974 for review). Interestingly, the selective peripheral vasodilation in our study was paralleled by a fall in MAP suggesting no compensatory rise in cardiac output in response to the E2-induced afterload reduction. The fall in MAP together with a lack of increase in HR is consistent with down- 
regulation of the baroreceptor setpoint. It is conceivable that the lower MAP is an important functional response to $\mathrm{E} 2$ as it reduces glomerular pressure and with it the pressure diuresis. Such an effect gives rise to enhanced sodium retention (Christy 1974, Hall 1990) eventually resulting in secondary hypervolemia. The latter again leads to a higher venous return and, by Frank Starling, leads to an increase in stroke volume and thus in cardiac output, changes that can be observed in pregnancy.

E2 is released by the corpus luteum in the cycle and after conception. In early pregnancy and pseudopregnancy, initial E2 levels remain low (Bridges 1984, Gallo 1985, Garland 1987, Smith 1975) in the rat, as well as in the guinea pig (Challis 1971), sheep (Rosenfeld 1976, and human (Mishell 1973). In rat pregnancy circulating plasma E2 levels increase gradually between days 10 and 18 postmating (Bridges 1984, Gallo 1985). It has been postulated that an E2 surge occurs on the afternoon of day 4 of rat pregnancy (Shelesnyak 1963, Yoshinaga 1969). This E2 surge seems essential for successful implantation (Shelesnyak 1963). To mimic the transient increase in E2 in early rat pregnancy (Shelesnyak 1963), the animals in our study received a bolus. However, the design of our study was such that no conclusion can be drawn with respect to a role of this E2 surge in inducing all initial hemodynamic adaptations to pregnancy. Interestingly, Magness (Magness 1993) reported that a loading dose of E2 is needed to induce cardiovascular changes after chronic E2 administration, otherwise the initiation of these responses is delayed by 1 week. They postulated that this priming was necessary to saturate the adipose and other tissue depots with the steroid hormone. We speculate that this initial E2 surge is necessary to initiate the hemodynamic adaptation and increase the sensitivity of the vasculature for vasodilating substances, e.g. nitric oxide. Several observations support the view that $\mathbb{E} 2$ might regulate nitric oxide synthases (NOS) (Goetz 1994, Weiner 1994b). It is not clear whether $\mathrm{E} 2$ has a direct or an indirect effect on nitric oxide synthase induction: direct via NO gene expression, indirect by increasing flow and thus inducing NO via increased shear stress, or a combination of both (Learmont 1996). In vitro studies in sheep uterine and renal arteries showed that E2 significantly enhances the vasorelaxation in uterine arteries and the production of citrullines, but not in renal arteries (Veille 1996). Our results confirm the differential response between the two vascular beds. Pregnancy and pseudopregnancy are also associated with increased NO synthesis and shear stress-mediated NO-dependent relaxation (Conrad 1989, Learmont 1996). It has been shown that treatment with the estro- 
gen receptor antagonist tamoxifen in guinea pig pregnancy, decreases NOS activity to nonpregnant values in the heart (Weiner 1994b). This suggests an important role for estrogens in the NO-mediated vasodilated state of pregnancy. However, blockade of NO by L-NAME fails to completely prevent myogenic and flow-mediated endothelium-dependent vasodilatation in pregnant rats (Veille 1996), as well as acetyl-choline mediated relaxation in pregnant women (McCarthy 1994). This suggests that other relaxing factors (e.g. endotheliumderived hyperpolarizing factor) are involved (McCarthy 1994, Veille 1996).

By measuring blood flow simultaneously in an artery supplying the kidney and an artery supplying mostly skeletal muscle and skin, we were able to identify the specificity of a possible vasorelaxing effect of E2. Our data show that E2 does not raise renal blood flow, a normally occurring phenomenon in early pregnancy (Baylis 1979/1980, Davis 1989). Therefore, we conclude that E2 alone is not directly responsible for the initial pregnancy-related vasorelaxation. However, it is possible that the initial pregnancy-related vasorelaxation develops gradually after sustained E2 exposure, probably in concert with some other vasoactive (luteal or decidual) factor. Whether E2 also acts through other vasodilators, such as endothelium-derived hyperpolarizing factor, remains to be investigated. 


\section{Chapter 8}

General discussion 
Chapter 8 


\section{General discussion}

Pregnancy is characterized by the occurrence of important maternal physiologic adaptations. The onset of, and interrelationship between the profound gestational changes in the cardiovascular system, renal function and volume homeostasis are poorly understood. Understanding the mechanisms that lead to these adaptations is important, since pregnancies complicated by intra-uterine growth restriction and/or preeclampsia are preceded by maternal hemodynamic maladaptations in the immediate postimplantation period. Unfortunately, data obtained in this period of pregnancy are scarce, partly because of the ethical constraints on studies that require serial invasive measurements in early human pregnancy. The objective of this thesis was in the first place to describe the hemodynamic and volume changes in early rat pregnancy. On the basis of these observational studies, two studies were designed to further explore the underlying mechanism. The rat was considered an appropriate model for these studies, since adaptations in the last week of rat pregnancy resemble those in the third trimester of human pregnancy. All experiments were performed in conscious rats, adequately recovered from surgery, to exclude hemodynamic effects of anaesthesia and/or surgical stress. The methods used are known to have good reproducibility and the study design was always longitudinally, thus limiting the impact of biological variation on experimental results.

To determine the onset of the systemic hemodynamic changes in rat pregnancy, we measured cardiac output, stroke volume, heart rate, mean arterial pressure, and hematocrit longitudinally from early pregnancy onward (chapter 2). Measurements were performed daily on days 4 to 12 , and on days 14, 18 and 20 of pregnancy. Day 4 was chosen as the first measurement day to ensure adequate recovery of the rat from surgery. Furthermore, data collected on days 4 and 5 represent pre-implantation reference values which offer the possibility to discern a time-sequence between implantation (day 6) and the onset of hemodynamic changes. There is general agreement about the hemodynamic changes in the last week of rat pregnancy (Ahokas 1983, Bruce 1976, Gilson 1992, Lundgren 1979). However, the results in mid-pregnancy are conflicting (Ahokas 1983, Dowell 1993, Gilson 1992). Therefore, we also performed measurements on days 14, 18 and 20 to compare our results with those found by others. We chose to use electromagnetic flow probes around the ascending aorta to measure cardiac output, stroke volume and myocardial performance indices, since this method has been 
used widely and validated properly (Montani 1995, Pfeffer 1973 Smith 1987, Smith 1979, Smith 1980, Smits 1982). The most important finding of our first study is a gradual rise in cardiac output, primarily due to a rise in stroke volume, that starts immediately after implantation, between days 6 and 8 . The increase in stroke volume in early pregnancy may result from a rise in preload, a fall in afterload, an increase in cardiac contractility, or any combination of these three options. The rise in peak aortic flow and aortic flow acceleration in our study, indicators of myocardial performance, are consistent with improved myocardial contractility. However, our in vivo study design does not differentiate between intrinsic contractile changes of the cardiac muscle and contractile changes as a result of the altered preload and afterload. Improved intrinsic myocardial performance has been demonstrated in late pregnant rats in isolated working hearts (Buttrick 1987). A fall in afterload in early pregnancy, secondary to the fall in peripheral vascular resistance, can be expected to raise stroke volume. Whether the fall in afterload in early pregnancy is achieved by peripheral vasorelaxation only, or by a combination of the latter with an increased aortic compliance, is still unsettled. It is conceivable that aortic compliance increases in pregnancy, by aortic smooth muscle relaxation and/or by aortic dilation, e.g. clue to a higher NO release triggered by the rise in aortic flow. To evaluate whether increased aortic compliance contributes to the early pregnancy adaptations, we measured aortic compliance using a recently developed noninvasive ultrasound technique (chapter 3). In addition, the contribution of changes in structural aortic wall properties was assessed by morphometry. On the basis of the results of this study we conclude that aortic compliance and distensibility increase between days 4 and 8 of rat pregnancy, without concomitant change in aortic diameter. These functional changes are not related to structural changes in aortic wall mass, confirming previous studies (Awal 1995). This is further supported by the observation that the total amount of collagen has not changed in the aorta of term pregnant rats (Foidart 1978).

The fall in afterload in early rat pregnancy is associated with increased plasma renin activity on day 6 (Conrad 1989a). This raises the question whether the increased stroke volume is inadequate to meet the raised peripheral flow demands, which would imply that the vascular bed is relatively underfilled. If early pregnancy is considered to be a state of vascular underfill, HR can be expected to increase in response to a higher sympathetic tone. However, in rat pregnancy, HR increases in late pregnancy only (chapter 2). The lack of a rise in 
HR in early pregnancy might be explained by a fall in sympathetic tone or a rise in vagal activity. To evaluate whether autonomic nervous function and baroreceptor reflex sensitivity change in early pregnancy, blood pressure and heart variability were studied in chronically instrumented conscious rats (chapter 4). In this study, autonomic nervous activity and baroreceptor reflex sensitivity did not change. Thus, it seems not likely that the vascular bed is being sensed as underfilled. Apparently, the high-flow low-resistance circulation in early pregnancy, with its higher demand for intravascular volume, develops so slow, that the concomitant plasma volume expansion is adequate at any time to preserve the mean circulatory filling pressure and with it, venous return. This is supported by the unchanged plasma AVP levels observed by others (Barron 1988, Davison 1984, Davison 1988, Dürr 1981). In this concept, the raised plasma renin activity is more likely to result from changes other than a relative underfill. The increase in circulating levels of progesterone may contribute to the increase in plasma renin activity through its natriuretic action, which appears to be due to competitive inhibition of aldosterone in the nephron (Oparil 1975). It is conceivable that the production of renin is reset to a higher level in order to induce sodium retention throughout pregnancy. It should be taken into account that the measurement of plasma renin activity does not adequately reflect the absolute changes in renin secretion but depends on changes in renin substrate and the methodological errors using this indirect assay (Derkx 1987, Wilson 1980). The observed general refractoriness to vasoconstrictors (Nakamura 1988, Paller 1984) may be crucial to maintain the vasodilated state of pregnancy, in spite of increased angiotensin II levels. The latter results in increased aldosterone levels, which further promotes sodium and water retention (Phippard 1986, Dürr 1981, Garland 1987, Wilson 1980).

Plasma volume expansion, as suggested by the fall in hematocrit between days 6 and 8 in chapters 2 and 3 , will raise preload, and thus increase stroke volume and cardiac output. Plasma volume expansion in early pregnancy can be accomplished by fluid retention and/or a shift of extracellular fluid toward the vascular compartment. Previous studies have shown that neither fluid intake nor urine output change in the first week of pregnancy, indicating that plasma volume expansion in early pregnancy is not achieved by fluid retention (Atherton 1981, Atherton 1982b). However, fluid balance studies using metabolic cages to quantify volume input and output may not be accurate enough to discern a small fluid retention. Interestingly, in a previous study, it was demonstrated that PV expansion takes 
place even when the rat is kept on a zero sodium intake (Baylis 1990). This observation supports PV expansion by a fluid shift rather than by fluid retention. However, in that study only PV was measured and conclusions were drawn without concomitant information on fluid balance or changes in the extracellular fluid compartment. To evaluate whether plasma volume expansion in early pregnancy is accomplished by redistribution or by expansion of the extracellular fluid volume, we measured simultaneously plasma volume, extracellular volume and total body water (chapter 5). In this study, plasma volume increased between days 6 and 12 , in concert with a comparable rise in both total body water and extracellular fluid volume. The concomitant increase in the 3 variables suggests that the increase in plasma volume observed in early pregnancy results from expansion of the extracellular volume by fluid retention.

In summary, the studies in the first part of this thesis show that cardiac output increases between days 6 and 8 of rat pregnancy, by a rise in stroke volume. This rise in stroke volume, in turn, is triggered by a fall in afterload in early pregnancy, due to a combined fall in vascular resistance and a rise in aortic compliance. Whether the increased myocardial activity develops secondary to a fall in afterload or represents an independent effect of pregnancy, can not be derived from our studies. Also preload contributes to the rise in stroke volume and cardiac output by a gradual rise in plasma volume accomplished by volume retention.

Studies in human and baboon also indicate a selective gradual increase in stroke volume, accompanied by a rise in left ventricular mass, left ventricular wall thickness and cardiac contractility (Duvekot 1993, Phippard 1986, Robson 1989). The apparently balanced changes in cardiac and aortic dynamics raise the question whether they are causally related or develop independently in response to some overall relaxing factor. In pregnancy, the compliance of the entire vascular bed may be increased (chapter 3). A larger left ventricular size (Hart 1985, Morton 1984) without increase in filling pressure (Davis 1989) in guinea pig pregnancy, can be interpreted as an effect of an increased cardiac compliance. Assuming the development of a higher overall vascular compliance in pregnancy, and with it a decreased peripheral resistance, we postulate that one of the first effects of the changed vascular dynamics will be sodium and water retention to maintain vascular filling pressure. On the basis of the results of our study on autonomic control, we assume that the changes in the cardiovascular system develop so slow that the concomitant volume retention is adequate at any time to preserve the vascular filling state. This slow rate of change may represent an important characteris- 
tic of hemodynamic adaptation to pregnancy as a too rapid change may trigger a stress response with a higher sympathetic tone, and with it, a compromise of the blood supply to the implantation sites.

The second part of the thesis focuses on the factors responsible for the initiation of the hemodynamic adaptations to pregnancy. The observational studies provided evidence for the initiation of the hemodynamic changes by day 6 , at the time of, or shortly after implantation. The endocrine changes in the first 10 days of pregnancy and pseudopregnancy are almost similar. The two conditions differ from each other by the presence or absence of the trophoblast. Therefore, pseudopregnant rats were studied to differentiate between maternal and fetoplacental factors (chapter 6). The most important finding in our study is that pseudopregnant and pregnant rats show almost identical hemodynamic adaptations over the first 10 days. These results suggest that the trophoblast is not involved in the initial development of the cardiovascular changes. During the first 8 days of pseudopregnancy the circulating levels of progesterone, estradiol-17B (E2) and prolactin resemble closely those in normal rat pregnancy (chapter 1). The similarity in endocrine profille in early rat pregnancy and pseudopregnancy supports the view that at least one of these 3 hormones is involved in triggering the hemodynamic adaptation. Estradiol $-17 B$ is a potential candidate because of its vasodilating properties (Magness 1993, Rosenfeld 1976, Weiner 1994b). In early rat pregnancy and pseudopregnancy, initial E2 levels remain low except for an E2 surge on the afternoon of day 4 postmating. To evaluate whether this E2 surge contributes to the initial renal and hemodynamic adaptations in pregnancy, we investigated the acute effect of estradiol-17B on skeletal and renal blood flow in awake, ovariectomized rats. However, the differential response between the hindquarter and kidney suggests that E2 alone can not be kept responsible for the initial gestational fall in vascular tone in both vascular beds. Since also the vasoactive properties of both progesterone (Hart 1985, Nakamura 1988) and prolactin (Bryant 1973, Kaufman 1981, Mills 1981) in physiologic concentrations are modest, it seems highly unlikely that the induction of hemodynamic adaptation in early pregnancy can be explained by some simple dose-response relationship between the three luteal hormones and the cardiovascular system. Based on the hemodynamic data in pregnancy and pseudopregnancy, and the endocrine data in early pregnant and pseudopregnant rat, we speculate that one of the three luteal hormones, or a combination, triggers the development of peripheral arterial vasodilation only after they have circulated for at least 6 days. In this 
respect, we assume that the relaxation of the entire cardiovascular system in early pregnancy develops in a complex way in which the endocrine environment plays a mediating rather than initiating role. It is possible that the presence of a certain endocrine environment forms a prerequisite to enable the release of some still unknown potent vasodilating factor by the corpus luteum or the decidualized endometrium. This factor may act either directly on the vessel wall or indirectly by increasing the affinity of estradiol-17B to its receptor in the vessel wall. Since estradiol-17ß induces the expression of endothelial NO synthase (Weiner 1994b), estradiol-17B may contribute to the increased NO production and with it, to the vasodilated state of pregnancy. Such an indirect mechanism may explain the absence of any profound increase in estradiol-17B levels at the time of maximum hemodynamic changes. The initial vasorelaxation gives rise to afterload reduction leading to a high-flow low-resistance circulation and activation of volume retention. Blockade of NO release by L-NAME only partially blocks myogenic and flow-mediated endothelium-dependent vasodilatation in pregnant rats (Kim 1994, Pascoal 1995, Veille 1996), and acetyl-choline mediated relaxation in pregnant women (McCarthy 1994). This suggests that other relaxing factors (e.g. endothelium-derived hyperpolarizing factor) are involved. Recently, it has been shown in vitro that endothelium-dependent relaxation in nonpregnant women depends importantly on a hyperpolarizing vasodilator, acting at the vascular smooth muscle potassium channels (Pascoal 1996). In contrast, the augmented endothelium-dependent relaxation in omental vessels in human pregnancy was

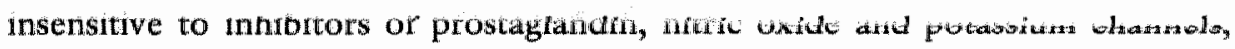
suggesting the presence of a yet unidentified endothelial vasoilator in pregnancy (Pascoal 1996). 
General discussion 
References 
Ahokas, R.A., G.D. Andersion, and J. Lipshitz. Cardiac output and uteroplacental blood flow in diet-restricted and diet-repleted pregnant rats. Am. J. Obstet. Gynecol. 146:6-13, 1983.

Ahokas, R.A., B.M. Sibai, and G.D. Anderson. Lack of evidence of a vasodepressor role for relaxin in spontaneously hypertensive and normotensive pregnant rats. Am. J. Obstet. Gynecol. 161: 618-622, 1989.

Alexander, E.A., S. Churchill, and H.H. Bengele. Renal hemodynamics and volume homeostasis during pregnancy in the rat. Kidney Int. 18: 173-178, 1980.

Atherton, J.C. Glomerular filtration rate and salt and water reabsorption during pregnancy in the conscious rat. J. Physiol. 334: 493-504, 1983.

Atherton, J.C., D. Bu'Lock, and S.C. Pirie. The effect of pseudopregnancy on glomerular filtration rate and salt and water reabsorption in the rat. J. Pbystol. 324: 11-20, 1982a.

Atherton, J.C., J.M. Dark, H.O. Garland, M.R.A. Morgan, J. Pidgeon, and S. Soni. Changes in water and electrolyte balance, plasma volume and composition during pregnancy in the rat. J. Pbysiol. 330: 81-93, 1982b.

Atherton, J.C., and S.C. Pirie. The effect of pregnancy on glomerular filtration rate and salt and water reabsorption in the rat. J. Pbysiol. 319: 153-164, 1981.

Awal, M.A., M. Matsumoto, and H. Nishinakagawa. Morphometrical changes of the arterial walls of main arteries from heart to the abdomino-inguinal mammary glands of rat from virgin through pregnancy, lactation and post-weaning. J. Vet. Med. Sci. 57: 251-256, 1995.

Bambra, C.S., and S. Gombe. The role of placental gonadotrophins (PMSG and hCG) in pregnancy in the rat. J. Reprod. Fert. 53: 109-115, 1978.

Barron, Volume homeosrasis during pregnancy in the rat. Am. J. Kidney Dis. IX no4: 296-302, 1987 .

Barron, W.M., and M.D. Lindheimer. Osmoregulation in pseudopregnant and prolactintreated rats: comparison with normal gestation. Am. J. Physiol. 254: R478-R484, 1988.

Barron, W.M., J. Schreiber, and M.D. Lindheimer. Effect of ovarian sex stetroids on osmoregulation and vasopressin secretion in the rat. Am. J. Pbysiol. 250: E352-E361, 1986.

Barron, W.M., B.A. Stamoutsos, and M.D. Lindheimer. Role of volume in the regulation of vasopressin secretion during pregnancy in the rat. J. Clin. Invest. 73: 923-932, 1984. 
Bassenge, E., and R. Busse. Endothelial modulation of coronary tone. Prog. Cardiouasc. Dis. $30: 349-380,1988$.

Baylis, C. Effect of early pregnancy on glomerular filtration rate and plasma volume in the rat. Renal Pbysiol. Basel 2: 333-339, 1979/1980.

Baylis, C. Glomerular ultrafiltration in the pseudopregnant rat. Am. J. Pbysiol. 234: F300F305, 1982 .

Baylis, C. Renal effects of cyclooxygenase inhibition in the pregnant rat. Aw. J. Pbysiol. 253: F158-F163, 1987a.

Baylis, C. The determinants of renal hemodynamics in pregnancy. Am. J. Kidney Dis, vol IX no $4: 260-264,1987 \mathrm{~b}$.

Baylis, C. The mechanism of the increase in glomerular filtration rate in the twelve-day pregnant rat. J. Physiol. 305: 405-414, 1980.

Baylis, C., K. Badr, and R. Collins. Effects of chronic prolactin administration on renal hemodynamics in the rat. Endocrinology 117: 722-729, 1985 a.

Baylis, C., and R.C. Blantz. Tubuloglomerular feedback activity in virgin and 12-day pregnant rats. Am. J. Physiol. 249: F169-F173, 1985 b.

Baylis, C, and B.M. Brenner. The physiological determinants of glomerular ultrafiltration. Rev. Physiol. Biochem. Pharmacol. 80: 1-46, 1978.

Baylis, C., and K. Engels. Adverse interactions between pregnancy and a new model of systemic hypertension produced by chronic blockade of endothellal derived relaxing factor (EDRF) in the rat. Clin. Exper. Hyper. in Pregnancy, B11 (2\&3): 117-129, 1992.

Baylis, C., and K. Munger. Persistence of maternal plasma volume expansion in midterm pregnant rats maintained on a zero sodium intake: evidence that early gestational volume expansion does not tequire renal sodium retention. Clin and Exper.Hyper in pregnancy B9(3), 237-247, 1990.

Bertinieri, G., M. Di Rienzo, A. Cavallazzi, A.U. Ferrari, A. Pedotti, and G. Mancia. Evaluation of baroreceptor reflex by blood pressure monitoring in unanesthetized cats. Am. J. Pbysiol. H377-H383, 1988.

Biswas, S., and C.H. Rodeck. Plasma prolactin levels during pregnancy. Br. J. Obstet. Gynaecol. 83: 683, 1976 . 
Bridges, R.S. A quantitative analysis of the roles of dosage, sequence, and duration of estradiol and progesterone exposure in the regulation of maternal behavior in the rat. Endocrinology 114: 930-940, 1984.

Bruce, N.W. The distribution of blood flow to the reproductive organs of rats near term. J. Reprod. Fert: 46: 359-362, 1976.

Bryant, E.E., B.H. Douglas, and A.D. Ashburn. Circulatory changes following prolactin administration. Am. J. Obistet. Gynecol. 115: 53-57, 1973.

Buckberg, G.D., J.C. Luck, D.B. Payne, J.L.E. Hoffman, J.P. Archie, and D.E. Fixler. Some sources of error in measuring regional blood flow with radioactive microspheres. J. Appl. Physiol. 31(4): 598-604, 1971.

Buelke-Sam, J., C.J. Nelson, R.A. Byrd, and J.F. Holson. Blood flow during pregnancy in the rat: I. Flow patterns to maternal organs. Teratology 26: 269-277, 1982a.

Buelke-Sam, J., J.F. Holson, and C.J. Nelson. Blood flow during pregnancy in the rat: II. Dynamics of and litter variability in uterine flow. Teratology 26: 279-288, $1982 \mathrm{~b}$.

Buttrick, PM., T.F. Schaible, A. Malhotra, S. Mattioli, and J. Scheuer. Effects of pregnancy on cardiac function and myosin enzymology in the rat. Am. J. Physiol. 252: H846H850, 1987 .

Capeless, E.L., and J.F. Clapp. Cardiovascular changes in early phase of pregnancy. Am. J. Obstet. Gynecol. 161: 1449-1453, 1989.

Castro, L.C., Arora, C., Parvez, S., Parvez, H., Valenzuela, G., and C.J. Hobel. Plasma atrial natriuretic peptide levels during the rat estrous cycle, pregnancy, and puerperium. Am. J. Obstet. Gynecol. 160: 15-19, 1989.

Castro, L.C., C.J. Hobel, and J. Gornbein. Plasma levels of atrial natriuretic peptide in normal and hypertensive pregnancy: a meta-analysis. Am. J. Pbysiol. 171: 1642-1651, 1994.

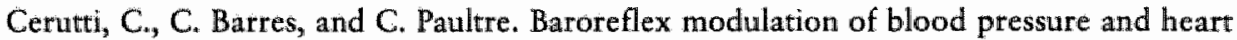
rate variabilities in rats: assessment by spectral analysis. Am. J. Pbysiol. 266: H1993H2000, 1994.

Cerutti, C., M.P. Gustin, C.Z. Paultre, M. Lo, C. Julien, M. Vincent, and J. Sassard. Autonomic nervous system and cardiovascular variability in rats: a spectral analysis approach. Am. J. Physiol. 261: H1292-H1299, 1991.

Cha, S.C., G.W. Aberdeen, B.S. Nuwayhid, and E.W. Quillen. Influence of pregnancy on mean systemic filling pressure and the cardiac function curve in guinea pigs. Can. $\mathrm{J}$. Pbysiol. Pharmacol. 70: 669-674, 1992. 
Challis, J.R.G., R.B. Heap, and D.V. Illingworth. Concentrations of oestrogen and progesterone in the plasma of nonpregnant, pregnant and lactating guinea pigs. J. Endocr. 51 * $333-345,1971$.

Christy, N.P., and J.C. Shaver. Estrogens and the kidney. Kidney lint. 6: 366-376, 1974.

Churchill, S.E. Sodium handling during pregnancy in the rat. Kidney Imt. 14: 753, 1978.

Clapp, J.F., B.L. Seaward, R.H. Sleamaker, and J. Hiser. Maternal physiologic adaptations to early human pregnancy. Am. J. Obstet. Gynecol. 159:1456-1460, 1988.

Conrad, K.P. Renal hemodynamics during pregnancy in chronically catheterized, conscious rats. Kidney Int. 26: 24-29, 1984.

Conrad, K.P., S.A. Barrera, P.A. Friedman, and V.M. Schmidt. Evidence for attenuation of myo-inositol uptake, phosphoinositide turnover and inositol phosphate production in aortic vasculature of rats during pregnancy. J. Clin. Invest. 87: 1700-1709, 1991.

Conrad, K.P., and M.C. Colpoys. Evidence against the hypothesis that prostaglandins are the vasodepressor agents of pregnancy. J. Clin. Invest. 77: 236-245, 1986.

Conrad, K.P., G.M. Joffe, H. Kruszyna, R. Kruszyna, L.G. Rochelle, R.P. Smith, J.E. Chavez, and M.D. Mosher. Identification of increased nitric oxide biosynthesis during pregnancy in rats. FASEB $]$. 7: 566-571, 1993.

Conrad, K.P, P.M. Morganelli, T. Brinck-Johnsen, and M.C. Colpoys. The reninangiotensin system during pregnancy in chronically instrumented, conscious rats. Am. J. Obstet. Gynecol. 161: 1065-1072, 1989a.

Conrad, K.P., and R.D. Russ. Augmentation of baroreflex-mediated bradycardia in conscious pregnant rats. Am. J. Fhysiol. 262: R472-R477, 1992.

Conrad, K.P., and K.A. Vernier. Plasma level, urinary excretion, and metabolic production of cGMP during gestation in rats. Am. J. Pbysiol. 257: R847-R853, 1989b.

Crandall, M.E., and C.M. Heesch. Baroreflex control of sympathetic outflow in pregnant rats: effects of captopril. Am. J. Physiol. 258: R1417-R1423, 1990 .

Culebras, J.M., G.F. Fitzpatrick, M.F. Brennan, C.M. Boyden, and F.D. Moore. Total body water and the exchangeable hydrogen II. A review of comparative data from animals based on isotope dilution and desiccation, with a report of new data from the rat. Am. J. Physiol. 232: R60-R65, 1977.

Curran-Everett, D., K.G. Morris, jr, and L.G. Moore. Regional circulatory contributions to increased systemic vascular conductance of pregnancy. Am. J. Physiol. 261: H1842H1847, 1991 . 
Danielson, L.A., and K.P. Conrad. Acute blockade of nitric oxide synthase inhibits renal vasodilation and hyperfiltration during pregnancy in chronically instrumented conscious rats. J. Clin. Invest. 96: 482-490, 1995 .

Davis, L.E., A.R. Hohimer, G.D. Giraud, M.S. Paul, and M.J. Morton. Vascular pressurevolume relationships in pregnant and estrogen-treated guinea pigs. Am. J. Pbysiol. 257: R1205-1211, 1989.

Davison, J.M., E.A. Gilmore, J. Dür, G.L. Robertson, and M.D. Lindheimer. Altered osmotic thresholds for vasopressin secretion and thirst in human pregnancy. Am. J. Pbysiol. 246: F105-F109, 1984.

Davison, J.M., and M.C.B. Noble. Serial changes in 24 hour creatinine clearance during normal menstrual cycles and the first trimester of pregnancy. Br. J. Obstet. Gynaecol. 88: $10-17,1981 \mathrm{a}$.

Davison, J.M., E.A. Shiells, P.R. Philips, and M.D. Lindheimer. Serial evaluation of vasopressin release and thirst in human pregnancy. $J$. Clin. Invest. 81: 198-806, 1988.

Davison, J.M., M.B. Valloton, and M.D. Lindheimer. Plasma osmolality and urinary concentration and dilution during and after pregnancy: evidence that lateral recumbuncy inhibits maximal urinary concentrating ability. Br. J. Obstet. Gynaecol. 88: 472-479, $1981 \mathrm{~b}$.

De Greef, W.J., J. Dullaart, and G.H. Zeilmaker. Effect of hysterectomy on serum luteinizing hormone concentrations and on corpus lutem function in the rat. Endocrinology 98: $1228-1234,1976$

De Greef, W.J., J. Dullaar, and G.H. Zeilmaker. Serum concentrations of progesterone, luteinizing hormone, follicle stimulating hormone and prolactin in pseudopregnant rats: effect of decidualization. Endocrinology 101: 1054-1063, 1977.

Del Valle, G.O., M.D. Moscher, and K.P. Conrad. Serum immunoreactive erythropoietin and red blood cell mass during pregnancy in conscious rats. Am. J. Physiol. 265: R399R403, 1993.

Deng, Y., and S. Kaufman. Effect of pregnancy on activation of central pathways following atrial distension. Am. J. Physiol. 269: R552-R556, 1995.

Derkx, F.H.M. Human prorenin. Thesis, Rotterdam, 1987.

Devroey, P, M. Camus, G. Palermo, J. Smitz, L. Van Waesberghe, A. Wisanto, I. Wijbo, and A.C. Van Steigertem. Placental production of estradiol and progesterone after oocyte donation in patients with primary ovarian failure. Aw. J. Obstet. Gynecol. 162: 66-70, 1990. 
Di Rienzo, M., P. Castiglioni, G. Parati, G. Mancia, and A. Pedotti. Effects of sino-aortic denervation on special characteristics of blood pressure and pulse interval variability: a wide-band approach. Med. Biol. Eng. Comput. 34: 133-141, 1996.

Douglas, B.H., J.C. Harlan, H.G. Langford, and T.Q. Richardson. Effect of hyperwolemia and elevated arterial pressure on cirulatory dynamics of pregnant animals. Am. J. Obstet. Gynecol. 98: 889-893, 1967.

Dowell, R.T., and C.D. Kauer. Uteroplacental blood flow at rest and during exercise in late-gestation conscious rats. J. Appl. Physiol. 74(5): 2079-2085, 1993.

Dürr, J.A., B. Stamoutsos, and M.D. Lindheimer. Osmoregulation during pregnancy: evidence for resetting of the threshold for vasopressin secretion during gestation. J. Clin. Invest. $68: 337-346,1981$.

Duvekot, J.J., E.C. Cheriex, F.A.A. Pieters, P.P.C.A. Menheere, and L.I.H. Peeters. Early pregnancy changes in hemodynamics and volume homeostasis are consecutive adjustments triggered by a primary fall in systemic vascular tone. Am. J. Obstet. Gynecol. 169: 1382$1392,1993$.

Duvekot, J.J., E.C. Cheriex, F.A.A. Pieters, P.P.C.A. Menheere, H.J.A. Schouten and L.L.H. Peeters. Maternal volume homeostasis in early pregnancy in relation to fetal growth restriction. Obstet. Gynecol. 85: 361-367, 1995.

Duvekot, J.J., and L.L.H. Peeters. Maternal cardiovascular hemodynamic adaptation to pregnancy. Obstet. Gynecol. Surv. 49(12suppl): S1-S14, 1994.

Easterlïng, T.R., T.J. Benedetti, B.C. Schmucker, and S.P. Millard. Maternal hemodynamics in normal and pre-eclamptic pregnancies: a longitudinal study. Obstet. Gynecol. 76 : 1061-1069, 1990.

Ekholm, E.M.K., R.U. Erkkola, S.J. Piha, J.O. Jalonen, T.H. Metsälä, and K.J. Antila. Changes in autonomic cardiovascular control in mid-pregnancy. Clin. Physiol. 12: 527. 536,1992 .

Ekholm, E.M.K., S.J. Piha, K.J. Antila, and R.U. Erkkola. Cardiovascular autonomic reflexes in mid-pregnancy. Br. $J$. Obstet. Gynaecol. 100: 177-18.2, 1993.

Ekholm, E.M.K.y S.J. Piha, R.U. Erkkola, and K.J. Antila. Autonomic cardiovascular reflexes in pregnancy. A longitudinal study. Clin. Auton. Res. 1: 289-296, 1994.

Elkarib, A.O., H.O. Garland, and R. Green. Acute and chronic effects of progesterone and prolactin on renal function in the rat. J. Physiol. 337: 389-400, 1983. 
Eneroth-Grimfors, E., M. Westgren, M. Ericson, C. Ihrman-Sandahl, and L.E. Lindblad. Autonomic cardiovascular control in normal and pre-eclamptic pregnancy. Acta Obstet. Gynecol. Scand. 73: 680-684, 1994.

Farhat, M.Y., M.C. Lawigne, and P.W. Ramwell. The vascular protective effects of estrogen. FASEB J. 10: 615-624, 1996.

Foidart, J.M., G. Roriwe, and B. Nusgens. Aortic collagen synthesis during renal hypertension, pregnancy and hypertension during pregnancy in the rat. Biomedicine 28: 215-219, 1978.

Fowler, W.L., J.A. Johnson, J.A., K.D. Kurz, D.W. Zeigler, D.E. Dostal, and C.G. Payne. Body fluid wolumes in rats with mestranol-induced hypertension. Am. J. Physiol. 250: H190-H195, 1986.

Fritsch, J.M., D.L. Eckberg, L.D. Graves, and B.G. Wallin. Arterial pressure ramps provoke linear increases of heart period in humans. Am. J. Pbysiol. 251: R1086-R1090, 1986.

Gallo, R.V., E. Deworshak-Harvey, and A. Bona-Gallo. Pulsatile luteinizing hormone release during pregnancy in the rat. Endocrinology, $116: 2637-2642,1985$.

Ganguli, M.C, J.D. Smith, and L.E. Hanson. Sodium metabolism and its requirement during reproduction in female rats. J. Nutrition 99: 225-234, 1969.

Garland, H.O., J.C. Atherton, C. Baylis, M.R.A. Morgan, and C.M. Millne. Hormone profiles for progesterone, oestradiol, prolactin, plasma renin activity, aldosterone and corticosterone during pregnancy and pseudopregnancy in two strains of rat: correlation with renal studies. J. Endoct. 113: 435-444, 1987.

Gibson, J.G., and W.A. Evans. Clinical studies of the blood volume. I. Clinical application of a method employing the azo dye "Evans Blue" and the spectrofotometer. J. Clin. Invest. 16: $3011-316,1937$

Gilanyi, M., S. Simon, and A.G.B. Kovách. Interstitial fluid pressure changes in pregnant rats. Acta Pbysiol. Hung. 62(2): 131-138, 1983.

Gilson, G.J., M.D. Mosher, and K.P. Conrad. Systemic hemodynamics and oxygen transport during pregnancy in chronically instrumented, conscious rats. Am. J. Pbysiol. 263: H1911-H1918, 1992.

Goetz, R.M., I. Morano, T. Calovini, R. Studer, and J. Holtz. Increased expression of endothelial constitutive nitric oxide synthase in rat aorta during pregnancy. Biochem. Biophys. Res. Commun. 205: 905-910, 1994.

Goodlin, R.C., M.J. Niebauer, M.J. Holmberg, and I.M. Zucker. Mean circulatory filling pressure in pregnant rabbits. Am. J. Obstet. Gynecol. 148: 224-225, 1984. 
Guyton, A.C. Textbook of medical physiology, 8th edition. Philadelphia: Saunders, 1991.

Hall, J.E., H.L. Mizelle, D.A. Hildebrandt, and M.W. Brands. Abnormal pressure natriuresis. A cause or consequence of hypertension? Hypertension 15: 547-559, 1990.

Hamilton, H.F.H. The cardiac output in normal pregnancy. As determined by the Cournand right heart catheterisation technique. J. Obstet. Gynaecol. Br. Empire. 56: 548552,1949 .

Hart, M.V., J.D. Hosenpud, A.R. Hohimer, and M.J. Morton. Hemodynamics during pregnancy and sex steroid administration in guinea pigs. Am. J. Physiol. 249: R179-R185, 1985.

Hart, M.V., M.J. Morton, J.D. Hosenpud, and J. Metcalfe. Aortic function during normal human pregnancy. Awr. J. Obstet. Gynecol. 154: 887-891, 1986.

Hays, P.M., D.P. Cruiksbank, and L.J. Dunn. Plasma volume determination in normal and preeclamptic patients. Am. J. Obstet. Gynecol. 151: 958-966, 1985.

Haywood, J.R., R.A. Shaffer, C. Fastenow, G.D. Fink, and M.J. Brody. Regional blood flow measurement with pulsed Doppler flow meter in conscious rat. Am. J. Physiol. 241: $\mathrm{H} 273-\mathrm{H} 278,1981$

Hebel, R., and M.W. Stromberg. Anatomy and Embryology of the Laboratory Rat. Wörthsee, Federal Republic of Germany, Biomed, p. 231-257, 1986.

Hermsteiner, M.G.J., D.R. Zoltan, and J.F. Clapp III. Human chorionic gonadotrophin is a potent vasodilator of pre-arteriolar uterine and mesenteric vessels in pregnant and nonpregnant rats. J. Soc. Gynecol. Invest. 4 suppl: 1, 109A, 1997.

Hibbard, J., A. Poppas, C. Korcarz, R. Marcus, M. Lindheimer, and R. Lang. Aortic compliance in early pregnancy. Am. J. Obstet. Gynecol. 170: 286, 1994.

Hines, T., and W.M. Barron. Effect of sinoaortic denervation on pressor responses in pregnant rats. Am. J. Pbysiol. 262: R1 100-1105, 1992.

Hjemdahl, P, and P. Friberg. Biochemical assessment of sympathetic activity and prejunctional modulation of noradrenaline release in humans. $J$. Hypertension 14: 147-150, 1996.

Hoeks, A.P.G., P.J. Brands, F.A.M. Smeets, and R.S. Reneman. Assessment of the distensibility of superficial arteries. Ultrasound Med. Biol. 16: 121-128, 1990.

Hohmann, M., K. Mackey, S. Davidge, and M.K. McLaughlin. Venous remodeling in the pregnant rat. Clin. Exp. Hypertens. B10: 307-321, 1991. 
Humphreys, P.W, and N. Joels. Changes in cardiac output and total peripheral resistance during the carotid sinus baroreceptor reflex in the pregnant rabbit. J. Pbysiol. Lond. 272: $45-55,1977$.

Humphreys, $\mathrm{PW}$, and $\mathrm{N}$. Joels. Effect of pregnancy on pressure-volume relationships in circulation of rabbits. Am. J. Pbysiol. 267: R780-R785, 1994.

Humphreys, P.W., and N. Joels. The carotid sinus baroreceptor reflex in the pregnant rabbit. J. Pbysiol. Lond. 239: 89-102, 1974.

Hytten, F.E. A.M. Thomson, and N. Taggart. Total body water in normal pregnancy. J. Obstet. Gynaecol. Brit. Cwlth. 73: 553-561, 1966.

Jansakul, C., King, R.G., Boura, A.L.A., Brennecke S.P, and G.M. Handberg. Plasma levels of atrial natriuretic peptides during pregnancy and post partum in the rat. $J$. Endocr. $120 ; 113-117,1989$.

Janssen, B.J.A., J. Oosting, D.W. Slaaf, P.B. Persson, and H.A.J. Struijker-Boudier. Hemodynamic basis of oscillations in systemic arterial pressure in conscious rats. Am. $\mathrm{J}$. Pbysiol. 269: H62-H71, 1995.

Javeshghani, D., S. Mukaddam-Daher, L. Fan, Z. Guan, J. Gutkowska, B. Nuwayhid, and E.W. Quillen. Control of atrial natriuretic factor by right and left atrial distension in pregnant sheep. Am. J. Pbysiol. 268: R1411-R1417, 1995.

Julien, C., Z - Q. Zhang, C. Cerutti, and C. Barrès. Hemodynamic analysis of arterial pressure oscillations in conscious rats. J. Auton. Nerv. Syst. 50: 239-252, 1995.

Katz, A.I., and M.D. Lindheimer. Renal handling of acute sodium loads in pregnancy $A m$. J. Physiol. 225:696-699, 1973.

Kaufman, $S$., and $Y$. Deng. Renal tesponse to atrial stretch during pregnancy in conscious rats. Am. J. Pbysiol. 265: R902-R906, 1993.

Kaufman, S., Y. Deng, and W. Thai. Influence of pregnancy on ANF release from isolated atria. Am. J. Physiol. 266: R1605-R1609, 1994.

Kaufman, S., B.J. Mackay, and J.Z. Scott. Daily water and electrolyte balance in chronically hyperprolactinaemic rats. J. Pbysiol. 321: 11-19, 1981.

Kaufman, S., and $K$. Novak. Effects of pregnancy, estradiol and progesterone on plasma ANF levels and the pressor response to angiotensin II (AII). FASEB J. 5: A1414, 1991.

Kim, T.H., C.P. Weiner, and L.P. Thompson. Effect of pregnancy on contraction and endothelium-mediated relaxation of renal and mesenteric arteries. Am. J. Physiol. 267: H41-H47, 1994. 
Kirksey, A., and R.L. Pike. Some effects of high and low sodium intakes during pregnancy in the rat. J. Nutrition 77:33-42, 1962.

Langille, B.L., M.P. Bendeck, and F.W. Keeley. Adaptations of carotid arteries of young and mature rabbits to reduced carotid blood flow. Am. J. Physiol. 256: H931-H939, 1989.

Langille, B.L., R.D. Brownlee, and S.L. Adamson. Perinatal aortic growth in lambs: relation to blood flow changes at birth. Am. J. Physiol. 259: H1247-H1253, 1990.

LaSala, G.B., O. Gaddi, G. Bruno, L. Brandi, M. Cantarelli, V. Salvatore, M.G. Torelli, and D. Dall'Asta. Noninvasive evaluation of cardiovascular hemodynamics and during multiple follicular stimulation, late luteal phase and early pregnancy. Fertil Steril. 51: 796-802, 1989.

Learmont, J.G., A.P. Cockell, G.A. Knock, and L. Poston. Myogenic and flow-mediated responses in isolated mesenteric small arteries from pregnant and nonpregnant rats. Am. J. Obstet. Gynecol. 174: 1631-1636, 1996.

Leduc, L., N. Wasserstrum, T. Spillman, and D.B. Cotton. Baroreflex function in normal pregnancy. Am. J. Obstet. Gynecol. 165: 886-890, 1991.

Lesser, G.T., Deutsch, S. and J. Markofsky. Fat-free mass, toral body water, and intracellular water in the aged rat. Am. J. Physiol. 238: R82-R90, 1980.

Lichton, I.J. Salt saving in the pregnant rat. Am. J. Physiol. 201: 765-768, 1961.

Lin, A.L., and S.A. Shain. Estrogen-mediated cytoplasmic and nuclear distribution of rat cardiovascular estrogen receptors. Arteriosclerosis 5: 668-677, 1985.

Lindhard, J. Uber das Minutenvolume des Herzens bei Ruhe und bei Muskelarbeit. Pflugers Arch. 161: 223-253, 1915.

Longo, L.D. Maternal blood volume and cardiac output during pregnancy: a hypothesis of endocrinologic control. Am. J. Physiol. 245: R720-R729, 1983.

Lundgren, Y., K. Karlsson, and U. Ljungblad. Circulatory changes during pregnancy in spontaneously and renal hypertensive rats. Clin. Sci. 57: 337S-339S, 1979.

Lyons, T.P. and M.L. Riedesel. Glycerol-induced hyperhydration: Its effects on fluid compartments in the rat. Life Sciences 53: 1779-1987, 1993.

Mabie, W.C., T.G. DiSessa, L.G. Crocker, B.M. Sibai, and K.L. Arheart. A longitudinal study of cardiac output in normal human pregnancy. Am. J. Obstet. Gynecol. 170: 849 $856,1994$. 
Magness, R.R., C.R. Parker, and C.R. Rosenfeld. Systemic and uterine responses to chronic infusion of estradiol-17B. Am. J. Pbysial. 265: E690-E698, 1993.

Magness, R.R., and C.R. Rosenfeld. Local and systemic estradiol-17B: effects on uterine and systemic vasodilation. Am. J. Physiol. 256: E536-E542, 1989.

Masilamani, 5., Castro, L., and C. Baylis. Pregnant rats are refractory to the natriuretic actions of atrial natriuretic peptide. Aw. I. Pbysiol. 267: R1611-R1616, 1994.

Massicotte, G., A. Parent, and J. St-Louis, Blunted response to vasoconstrictors in mesenteric wasculature but not in portal vein of spontaneously hypertensive rats treated with relaxin (42857). Proc. Soc. Exp. Biol. Med. 190: 254-259, 1989.

Matthews, B.F., and D.W. Taylor. Effects of pregnancy on inulin and para-aminohippurate clearances in the anaesthetized rat. J. Pbysiol. 151: 385-389, 1960.

McCarthy, A.L., P. Taylor, J. Graves, S.K. Raju, and L. Poston. Endothelium-dependent relaxation of human resistance arteries in pregnancy. Am. J. Obstet. Gynecol. 171: 1309 . $1315,1994$.

McGill, H.C., and P.J. Sheridan. Nuclear uptake of sex steroid hormones in the cardiovascular system of the baboon. Circ. Res. 48: 238-244, 1981.

Melone, P.J., P.J. Meis, and D.A. Blizard. Circadian thythms of heart rate and mean arterial pressure in chronically instrumented pregnant rats. Am. J. Obstet. Gynecol. 165: 758$763,1991$.

Miller, A.E., and G.D. Riegle. Serum progesterone during pregnancy and pseudopregnancy and gestation length in the aging rat. Biol. Reprod. 22: 751-758, 1980.

Mills, D.E., M.T. Buckman, and G.T. Peake. Effects of prolactin administration and suppression on blood pressure and body fluid compartments in the rat. Endocrinology 109: $1590-1596,1981$.

Mishell, D.R., I.H. Thorneycroft, Y. Nagata, T. Murata, and R.M. Nakamura. Serum gonadotropin and steroid patterns in early human gestation. Am. J. Obstet. Gynecol. 117: $631-639,1973$.

Miyamoto, S., H. Shimokawa, H. Sumioko, A. Touno, and H. Nakano. Circadian rythm of plasma atrial natriuretic peptide, aldosterone, and blood pressure during the third trimester in normal and preeclamptic pregnancies. Am. J. Obstet. Gynecol. 158: 393-399, 1988 .

Moe, G.W., I. Legault, and K.L. Skorecki. Control of extracellular fluid volume and pathophysiology of edema formation. In: the kidney, eds. Brenner, B.M. and Rector, F.C., W.B. Saunders company, Philadelphia 1991, pp. 623-676. 
Molnăr, M., and F. Hertelendy. NZ-Nitro-L-arginine, an inhibitor of nitric oxide synthesis, increases blood pressure in rats and reverses the pregnancy-induced refractoriness to vasopressor agents. Awt. J. Obstet. Gynecol. 166: 1560-1567, 1992.

Molnár, M., T. Sütö, T. Tóth, and F. Hertelendy. Prolonged blockade of nitric oxide synthesis in gravid rats produces sustained hypertension, proteinuria, thrombocytopenia, and intrauterine growth retardation. Am. J. Obstet. Gynecol. 170: 1458-1466, 1994.

Moncada, S., and A. Higgs. The L-arginine-nitric oxide pathway. N. Engl. J. Med. 329: 27: 2002-2012, 1993.

Montani, J., H.L. Mizelle, B.N. van Vliet, and T.H. Adair. Advantages of continuous measurement of cardiac output $24 \mathrm{~h}$ a day. Am. J. Physiol. 269: H696-H703, 1995.

Morton, M., H. Tsang, R. Hohimer, D. Ross, K. Thornburg, J. Faber, and J. Metcalfe. Left ventricular size, output, and structure during guinea pig pregnancy. Am. J. Physiol. 246: R40-R80, 1984.

Mukaddam-Daher, S., J. Gutkowska, B.S. Nuwayhid, and E.W Quillen, jr. Metabolic clearance of atrial natriuretic factor in ovine pregnancy. Am. J. Pbysiol. 267: R1413R1420, 1994.

Myers, S.A., and H. Tseng. A longitudinal study of cardiac output in unstressed pregnant guinea pigs. Am. J. Pbysiol. 248: R698-R701, 1985.

Nadel, A.S., B. J. Ballermann, S. Anderson, and B. Brenner. Interrelationships among atrial peptides, renin, and blood volume in pregnant rats. Am. J. Pbysiol. 254: R793-R800, 1988.

Nakamura, T., K. Matsui, I. Masaharu, T. Yoshimura, N. Kawasaki, S. Fujisaki, and H. Okamura. Effects of pregnancy and hormone treatments on pressor response to angiotensin II in conscious rats. Am. J. Obstet. Gynecol. 159: 989-995, 1988.

Nichols, W.W., and M.F. O'Rourke. McDonald's Blood Flow in Arteries, third edition. London: Edward Arnold, 1990.

Ogle, T.F, and J.I. Kitay. Ovarian and adrenal steroids during pregniancy and the oestrus cycle in the rat. J. Endocr. 74: 89-98, 1977.

Oosting, J., H.A.J. Struijker-Boudier and B.J.A. Janssen. Validation of a continuous baroreceptor reflex sensitivity index calculated from spontaneous fluctuations of blood pressure and pulse interval in rats. J. Hypertension, 15: 391-399, 1997.

Oparil, S., E.H. Ehrlich, and M.D. Lindheimer. Effect of progesterone on renal sodium handling in man: relation to aldosterone secretion and plasma renin activity. Clinical Science and molecular medicine 49: 139-147, 1975. 
Otsuki, Y. E. Okamoto, I. Iwata, E. Nishino, N. Mitsuda, M. Mori, T. Takagi, N. Sugita, and $\mathrm{O}$. Tanizawa. Changes in concentration of human atrial natriuretic peptide in normal pregnancy and toxaemia. f. Endoct. 114, 325-328, 1987.

Paller, M.S. Mechanism of decreased pressor responsiveness to ANG II, NE and vasopressin in pregnant rats. Am. J. Pbysiol. 247: H100-H108, 1984.

Paller, M.S., G. Gregorini, and T.F. Ferris. Pressor responsiveness in piseudopregnant and pregnant rats: role of maternal factors. Am. J. Pbysiol. 257: R866-R871, 1989.

Pang, C.Y., and H.R. Behrman. Relationship of luteal blood flow and corpus luteum function in pseudopregnant rats. Am. J. Physiol. 237 : E30-E34, 1979.

Pascoal, I.F., M.D. Lindheimer, C. Nalbantian-Brandt, and J.G. Umans. Contraction and endothelium-dependent relaxation in mesenteric microvessels from pregnant rats. Am. J. pbysiol. 269: H1899-H1904, 1995.

Pascoal, I.F, and J.G. Umans. Effect of pregnancy on mechanisms of relaxation in human omental microvessels. Hypertension 28: 183-187, 1996.

Pepe, G.J., and I. Rothchild. A comparative study of serum progesterone levels in pregnancy and in various types of pseudopregnancy in the rat. Endocrinology 95: 275-279, 1974.

Perlini, S., F. Giangregorio, M. Coco, A. Radaelli, P.L. Soldà, L. Bernardi, and A. U. Ferrari. Autonomic and ventilatory components of heart rate and blood pressure variability in freely behaving rats. Am. J. Physiol. 269: H1729-H1734, 1995.

Peysner, K., and M.L. Forsling. Effect of ovariectomy and treatment with ovarian steroids on vasopressin release and fluid balance in the rat. $J$. Endocrinol. 124: 277-284, 1990.

Pfeffer, M.A., and E.D. Frohlich. Hemodynamic and myocardial function in young and old normotensive and spontaneously hypertensive rats. Circ. Res. 32, suppl. 1: 28-35, 1973.

Phippard, A.F., J.S. Horvath, E.M. Glynn, M.G. Garner, P.J. Fletcher, G.G. Duggin, and D.J. Tiller: Circulatory adaptation to pregnancy-Serial studies of haemodynamics, blood wollume, renin and aldosterone in the baboon. J. Hypertens. 4: 773-779, 1986.

Pike, R.L. Sodium requirement of the rat during pregnancy. In: Hypertension in pregnan$c y$, eds. Lindheimer, M.D., Katz, A.I. and Zuspan, F.P. Wiley and Sons, New York, 1976, pp. $207-215$.

Quagliarello, J., N. Szlachter, B.G. Steinetz, L.T. Goldsmith, and G. Weiss. Serial relaxin concentrations in human pregnancy. Am. J. Obstet. Gynecol. 135:43, 1979. 
Reckelhoff, J.F., L. Samsell, and C. Baylis. Failure of an acute 10-15\% plasma volume expansion in the virgin female rat to mimic the increased glomerular filtration rate (GFR) and altered glomerular hemodynamics seen at midterm pregnancy. Clin, and exper. byper. in pregnancy B8: 533-549, 1989.

Reckelhoff, J.F., S.D. Yokota, and C. Baylis. Renal autoregulation in midterm and latepregnant rats. Am. J. Obstet. Gynecol. 166: 1546-1550, 1992.

Reneman, R.S., T. van Merode, P. Hick, A.M.M. Muytjens, and A.P.G. Hoeks. Age-related changes in carotid artery wall properties in men. Ultrasound Med. Biol. 12: 465-471, 1986.

Rizzoni, D., M. Castellano, E. Porteri, G. Bettoni, P. Muiesan, M.L. Muiesan, S.M. Guilini, A. Cinelli, M. Salvetti, and E.A. Rosei. Arterial spontaneous rhythmic contractile activity in humans and rats: spectral analysis and regulatory mechanisms. J. Hypertension 13: 1043-1052, 1995.

Robbe, H.W.J., L.J.M. Mulder, H. Rüddel, W.A. Langewitz, J.B.P. Veldman, and G. Mulder. Assessment of baroreceptor reflex sensitivity by means of spectral analysis. Hypertension 10: 538-543, 1987.

Robson, S.C., S. Hunter, R.J. Boys, and W. Dunlop. Serial study of factors influencing changes in cardiac output during human pregnancy. Am. J. Physiol. 256: H1060-H1065, 1989.

Rosenfeld, C.R. Distribution of cardiac output in ovine pregnancy. Am. J. Physiol. 232: H231-H235, 1977.

Rosenfeld, C.R., F.H. Morriss, F.C. Battaglia, E.L. Makowski, and G. Meschia. Effect of estradiol-17B on blood flow to reproductive and nonreproductive tissues in pregnant ewes. Am. J. Obstet. Gynecol. 124: 618-629, 1976 .

Sakai, K., T. Imaizumi, H. Maeda, H. Nagata, K. Tsukimori, A. Takeshita, and H. Nakano. Venous distensibility during pregnancy. Hypertension 24: 461-466, 1994.

Sanz, E., J.M. López Novoa, M. Linares, E. Digiuni, and C.A. Caramelo. Intravascular and interstitial fluid dynamics in rats treated with minoxidil. J. Cardiovasc. Pharmacol. 15: $485-492,1990$.

Schrier, R.W., and V.A. Briner. Peripheral arterial vasodilatation hypothesis of sodium and water retention in pregnancy: implications for pathogenesis of preeclampsia-eclampsia. Obstet. Gynecol. 77: 632-639, 1991.

Schrier, R.W., and J.A. Dürr. Pregnancy: an overfill or underfill state. Am. J. Kidney Dis. 4: 284-289, 1987. 
Shelesnyak, M.C., P.F. Kraicer, and G.H. Zeilmaker. Studies on the mechanism of decidualization. Acta endocrinologica 42: 225-232, 1963.

Sherwood, O.D., V.E. Crnekovic, W.L. Gordon, and J.E. Rutherford. Radioimmunoassay of relaxin throughout pregnancy and during parturition in the rat. Endocrinology 107 : $691-698,1980$.

Simon, A., J. Levenson, J. Bouthier, and B. Maarek. Haemodynamic basis of early modifications of the large arteries in borderline hypertension. J. Hypertens. 5: 179-184, 1987.

Slangen, B.F.M., I.C.M. Out, C.M. Verkeste, and L.L.H. Peeters. Hemodynamic changes in early pregnancy in chronically instrumented conscious rats. Am. J. Physiol. 270: H1779H1784, 1996.

Slangen, B.F.M., D.S. Van Ingen Schenaw, A.W. Van Gorp, J.G.R. De Mey, and L.L.H. Peeters. Aortic distensibility and compliance in awake pregnant rats. Am. J. Physiol.: 272* H1260-H1265, 1997.

Slater, A.J., N. Gude, L.J. Clarke, and W.A.W. Walters. Haemodynamic changes and left ventricular performance during high-dose oestrogen administration to male transsexuals. Br. J. Obst. Gynaecol. 93: 532-538, 1986.

Stnith, M.S., M.E. Freeman, and J.D. Neill. The control of progesterone secretion during the estrous cycle and early pseudopregnancy in the rat: prolactin, gonadotropin and steroid levels associated with rescue of the corpus luteum of psendopregnancy. Endocrinology 96: 219-226, 1975.

Smith, M.S., and J.D. Neill. Termination at midpregnancy of two daily surges of plasma prolactin initiated by mating in the rat. Endocrinology 98: 696-701, 1976.

Smith, T.L., T.G. Coleman, K.A. Stanek, and W.R. Murphy. Hemodynamic monitoring for $24 \mathrm{~h}$ in unanesthetized rars. Am. J. Physiol. 253: H1335-H1341, 1987.

Smith, T.L., and P.M. Hutchins. Anesthetic effects on hemodynamics of spontaneously hypertensive and Wistar-Kyoto rats. Am. J. Physiol. 238: H539-H.544, 1980.

Smith, T.L., and P.M. Hutchins. Central bemodynamics in the developmental stage of spontaneous hypertension in the unanesthetized rat. Hypertension 1: 508-517, 1979.

Smits, J.F.M., T.G. Coleman, T.L. Smith, C.M. Kasbergen, H. van Essen, and H.A.J. Struyker-Boudier. Antihypertensive effect of propanolol in conscious spontaneously hypertensive rats: central hemodynamics, plasma volume, and renal function during B-blockade with propanolol. J. Cardiovasc. Pharmacol. 4: 903-914, 1982. 
Smits, J.F.M., H. van Essen, C.M. Tijssen, and H.A.J Struyker-Boudier. Effects of ketanserin on hemodynamics and baroreflex effects in conscious spontaneously hypertensive rats. J. Cardiovasc. Phamacol. 10: 1-8, 1987.

Starkey, P.M. The buman placenta: a guide for clinicians and scientists. Oxford, England, 1993, chapter $12,362-414$.

St-Louis, J., and G. Massicotte. Chronic decrease of blood pressure by rat relaxin in spontaneously hypertensive rats. Life Sci 37: 1351-1357, 1985.

St-Louis, J., A. Parent, J. Gutkowska, J. Genest, and E.L. Schiffrin. Vasorelaxation and vascular binding sites for atrial natriuretic peptide in pregnant rats. Am. J. Physiol. 254: H1027-H1033, 1988.

Stauss, H.M., and K.C. Kregel. Frequency response characteristic of sympathetic-mediated vasomotor waves in conscious rats. Am. J. Physiol. 271: H1416-H1422, 1996.

Steegers, E.A.P., H.P.J.M. Van Lakwijk, J.H. Fast, A.W.H.J. Godschalx, H.W. Jongsma, T.K.A.B. Eskes, E.M. Symonds, and P:R. Hein. Atrial natriuretic peptide and atrial size during normal pregnancy. Br. J. Obstet. Gynecol. 98: 202-206, 1991.

Teeuw, A.H., and W. de Jong. Time course of decrease in blood pressure and in blood pressure response to vasopressor agents during pregnancy in the rat. Pflügers Arch. 341: 197$208,1973$.

Thapar, M., G.L. Kumari, T.G. Shrivastav, and P.K. Pandey. Hormonal control of implanation in guinea pigs. Steroids $52: 85-108,1988$.

Ueland, K., and J.T. Parer. Effects of estrogens on the cardiovascular system of the ewe.. Am. J. Obstet. Gynecol. 96: 402-406, 1966.

Umans, J.G., M.D. Lindheimer, and W.M. Barron. Pressor effect of endothelium-derived relaxing factor inhibition in conscious virgin and gravid rats. Am. J. Pbysiol. 259: F293$296,1990$.

Van Beek, E., J.H.M. Houben, P.N. van Es, C. Willekes, E.C.C.M. Korten, P.W. de Leeuw, and L.L.H. Peeters. Peripheral haemodynamics and renal function in relation to the menstrual cycle. Clinical Science 91: 163-168, 1996.

Van Gorp, A.W., D.S. van Ingen Schenau, A.P.G. Hoeks, H.A.J. Struyker Boudier, R.S. Reneman, and J.G.R. De Mey. Aortic wall properties in normotensive and hypertensive rats of various ages in vivo. Hypertension 26:363-368, 1995.

Van Gorp, A.W., D.S. van Ingen Schenau, J. Willigers, A.P.G. Hoeks, J.G.R. De Mey, H.A.J. Struyker Boudier, and R.S. Reneman. A technique to assess aortic distensibility and compliance in anesthetized and awake rats. Am. J. Physiol. 270: H780-H786, 1996. 
Van Kreel, B.K. An improved bromide assay for the estimation of extracellular water volume by capillary gas chromatography. Clin. Chim. Acta 231: 117-128, 1994.

Van Kreel, B.K., F. Van der Vegt, M. Meers, T. Wagenmakers, K. Westerterp, and A. Coward. Determination of total body water by a simple and rapid mass spectrometric method. J. Mass. Spectr. 31: 108-111, 1996.

Van Loan, M.D. L.E. Kopp, J.C. King, W.W. Wong, and P.L. Mayclin. Fluid changes during pregnancy: use of bioimpedance spectroscopy. J. Appl. Physiol. 78(3): 1037-1042, 1995.

Van Oppen, A.C.C., R.H. Stigter, and H.W. Bruinse. Cardiac output in normal pregnancy: a critical review. Obstet. Gynecol. 87: 310-318, 1996.

Veille, J"C., P. Li, J.C. Eisenach, A.G. Massmann, and J.P. Figueroa. Effects of estrogen on nitric oxide biosynthesis and vasorelaxant activity in sheep uterine and renal arteries in vitro. Am. J. Obstet. Gynecol. 174: 1043-1049, 1996.

Visser, W., and H.C.S. Wallenburg. Central hemodynamic observations in untreated preeclamptic patients. Hypertension 17: 1072-1077, 1991.

Vokes, T.J., N.M. Weiss, J. Schreiber, M.B. Gaskill, and G.L. Robertson. Osmoregulation of thirst and vasopressin during normal menstrual cycle. Am. J. Pbysiol. 254: R641-R647, 1988.

Walker, J., and H.O. Garland. Single mephron function during prolactin-induced pseudopregnancy in the rat. J. Endoct. 107: 127-131, 1985.

Walters, W.A.W., W.G. MacGregor, and M. Hills. Cardiac output at rest during pregnancy and the puerperium. Clin. Sci. 30: 1-11, 1966.

Wang, Y., J.T. Crofton, H. Liu, K. Sato, D.P. Brooks, and L. Share. Estradiol attenuates the antidiuretic action of vasopressin in ovariectomized rats. Am. J. Physiol. 268: R951-R957, 1995.

Weiner, C.P., R.G. Knowles, and S. Moncada. Induction of nitric oxide synthases early in pregnancy. Am. I. Obstet. Gynecol. 171: 838-843, 1994a.

Weiner, C.P., I. Lizasoain, S.A. Baylis, R.G. Knowles, I.G. Charles, and S. Moncada. Induction of calcium-dependent nitric oxide synthases by sex hormones. Prac. Natl. Acad. Sci. USA, 91: 5212-5216, 1994b.

Whittaker, P.G., and ' $\mathrm{T}$. Lind. The intravascullar mass of albumin during human pregnancy: a serial study in normal and diabetic women. Br. J. Obstet. Gynaecol. 100: 587-592, 1993. 
Wilson, M., A.A. Morganti, I. Zervoudakis, R.L. Letcher, B.M. Romney, P. von Oeyon, S. Papera, J.E. Sealey, and J.H. Laragh. Blood pressure, the renin-aldosterone system and sex steroids throughout normal pregnancy. Am. J. Med. 68: 97-104, 1980.

Woodiwiss, A.J., and G.R. Norton. Exercise-induced cardiac hypertrophy is associated with an increased myocardial compliance. J. Appl. Physiol. 78(4): 1303-1311, 1995.

Woods, L.L., H.L. Mizelle, and J.E. Hall. Autoregulation of renal blood flow and glomerular filtration rate in the pregnant rabbit. Am. J. Physiol. 252: R69-R72, 1987.

Xu, D., P. Martin, J. St. John, P. Tsai, S.N. Summer, M. Ohara, J.K. Kim, and R.W. Schrier. Upregulation of endothelial and neuronal constitutive nitric oxide synthase in pregnant rats. Am.J.Physiol. 271: R1739-R1745, 1996.

Yoshinaga, K., R.A. Hawkins, and J.F. Stocker. Estrogen secretion by the rat ovary in vivo during the estrous cycle and pregnancy. Endocrinology 85: 103-112, 1969.

Zarins, C.K., M.A. Zatina, D.P. Giddens, D.N. Ku, and S. Glagov. Shear stress regulation of artery lumen diameter in experimental atherogenesis. J. Vasc. Surgery 5: 413-420, 1987. 
Summary 
In early pregnancy, changes can be observed in the maternal cardiovascular system, renal function and in volume homeostasis. The onset of, and interrelationship between these adaptations is not clear. Understanding the physiological meaning and underlying mechanisms of these adaptations is important in the study of the pathogenesis of pregnancy complications, such as intra-uterine growth restriction and pre-eclampsia. These complications are not only preceded by defective placentation, but also by abnormal development of the hemodynamic, renal and volume changes.

The objective of this thesis was in the first place to describe the hemodynamic and volume changes in rat pregnancy, and in the second place to explore possible triggers for the initial changes.

CHAPTER 1 is a review of the literature with regard to the cardiovascular system, renal function and volume homeostasis in pregnancy. The endocrine changes in pregnancy and pseudopregnancy, and their possible role in the initiation of the cardiovascular, renal and volume adaptation to pregnancy are discussed.

CHAPTER 2 is an observational study to determine the onset of, and interrelationship between the cardiovascular adaptations in rat pregnancy. The most important finding of this study is an increase in cardiac output shortly after implantation (day 6), due to a rise in stroke volume. The observed rise in stroke volume seems to result from a fall in afterload, a rise in preload and a rise in cardiac contractility. The decrease in peripheral vascular resistance in early pregnancy is generally considered to be entirely responsible for the fall in afterload. However, a rise in aortic compliance may contribute to the decrease in afterload as well.

IN CHAPTER 3 we studied aortic compliance and distensibility in rat pregnancy. The main finding of this study is an increase in aortic compliance between days 4 and 10 of pregnancy, without a change in aortic diameter. Histological analysis of the aortic wall structure did not show any differences between late-pregnant and nonpregnant rats. We speculate that the rise in aortic compliance and distensibility, together with the decrease in total peripheral resistance, in early pregnancy serve to accommodate the increase in stroke volume, thus preventing a rise in blood pressure and arterial wall stress.

It has been reported that the high-flow low-resistance circulation of pregnancy is accompanied by an elevated plasma renin activity. This suggests that 
early pregnancy hemodynamics may be associated with a relative vascular underfill. However, in the rat, this relative vascular underfill does not trigger a rise in heart rate, indicating that reflex regulation of heart rate may have changed during early pregnancy.

IN CHAPTER 4 we evaluated whether changes in the autonomic control of heart rate and blood pressure occur in rat pregnancy. Spectral analysis of spontaneously occurring variations in blood pressure and heart rate did not reveal any changes in the autonomic nervous system in early pregnancy. Thus, the fall in afterload in early pregnancy in the rat does not seem to induce a state of relative vascular underfill, and develops without activation of the sympathetic nervous system.

IN CHAPTER 5 we investigated the changes in volume homeostasis in early pregnancy. Volume expansion can be expected to raise preload, and thus increase stroke volume and cardiac output. Plasma volume expansion in early pregnancy can be accomplished by fluid retention and/or a shift of extracellular fluid toward the vascular compartment. The gradual synchronous increase in total body water, extracellular fluid volume and plasma volume in this study suggests that the increase in plasma volume observed in early pregnancy results from expansion of the extracellular volume by fluid retention rather than by redistribution of the extracellular volume.

In the second part of the thesis we investigated some candidates that could be responsible for the initiation of the adaptations to pregnancy.

IN CHAPTER 6 the cardiovascular adaptations in psendopregnant rats were studied. The pseudopregnant and pregnant rats showed almost identical hemodynamic adaptations until day 9 . This suggests that, at least in the rat, the trophoblast is not essential for the initial hemodynamic adaptation to pregnancy. The similarity in endocrine profile in early rat pregnancy and pseudopregnancy supports the view that maternal hormones are involved in triggering the hemodynamic adaptation. Estradiol-17B is a potential candidate because of its wellknown vasodilating effects.

IN CHAPTER 7 we tested the hypothesis that the earliest rise in circulating estradiol-17B levels in rat pregnancy triggers the initial nonselective vasorelaxation of pregnancy, as will be reflected in higher blood flows to both the kidney and skeletal tissue. Our finding of an estradiol-17B-induced vasodilation 
in the vasculature supplying skeletal muscle and skin, but not the kidney, suggests that estradiol-17B alone is not directly responsible for the initial pregnancyrelated vasorellaxation.

In conclusion, the studies in this thesis show that cardiac output increases between days 6 and 8 of rat pregnancy, by a rise in stroke volume. This rise in stroke volume, in turn, is triggered by a fall in afterload in early pregnancy, due to a combined fall in peripheral vascular resistance and a rise in aortic compliance. Activation of volume retention will lead to a rise in plasma volume, most likely by salt and fluid retention. The increase in cardiac contractility could be secondary to the higher stroke volume that has to be ejected. However, a direct endocrine stimulus by e.g. estradiol-17ß can not be excluded. The trophoblast is not essential for the initiation of the cardiovascular and renal adaptations to pregnancy, since the adaptations are also observed in pseudopregnant rats. Estradiol$17 \AA$ alone is not the trigger for the generalized vasorelaxation in pregnancy, but may play a major role in mediating the release of potent vasodilators. 


\section{Samenvatting}


In de vroege zwangerschap worden veranderingen waargenomen in het hart-en vaatstelsel, de nierfunctie en de volumehomeostase bij de moeder. Het moment waarop deze aanpassingen beginnen en hun onderlinge relatie is niet duidelijk. Inzicht in de fysiologische betekenis van deze aanpassingen en het mechanisme dat eraan ten grondslag ligt is belangrijk voor de studie van de pathogenese van zwangerschapscomplicaties, zoals intra-uteriene groeivertraging en pre-eclampsie. Deze complicaties worden niet alleen voorafgegaan door afwijkende placentatie, maar ook door een afwijkend verloop van de hemodynamische, renale en volumeveranderingen.

Het doel van dit proefschrift is in de eerste plaats de hemodynamische en volumeveranderingen in de zwangere rat te beschrijven, en in de tweede plaats factoren die de initiële veranderingen induceren te exploreren.

HOOFDSTUK 1 bevat een literatuuroverzicht wan de veranderingen in het hart-en vaatstelsel, de nierfunctie en de volumehomeostase in de zwangerschap. De endocriene veranderingen in de zwangerschap en schijnzwangerschap, en hun mogelijke rol in de initiatie van de cardiovasculaire, renale en volumeadaptatie aan de zwangerschap, worden besproken.

HoofDSTUK 2 is een observationele studie om de aanvang en onderlinge relatie van de cardiovasculaire aanpassingen in de zwangerschap van de rat te bepalen. De belangrijkste bevinding van deze studie is een toename van het hartminuutvolume, kort na de implantatie van het embryo (dag 6), door een stijging van het slagvolume. De waargenomen stijging in het slagvolume lijkt het gevolg van een daling in de afterload, een stijging in de preload en een toename van de cardiale contractiliteit. In het algemeen wordt de afname in perifere vasculaire weerstand geheel verantwoordelijk gehouden voor de daling in afterload. Echter, een stijging in de arteriële compliance (=rekbaarheid) kan ook bijdragen aan de daling in afterload.

IN HOOFDSTUK 3 wordt de compliance en distensibiliteit van de aorta bestudeerd. De belangrijkste bevinding van dit hoofdstuk is een toename in de compliance van de aorta tussen dag 4 en 10 van de zwangerschap, zonder verandering in de aortadiameter. Histologische analyse van de structuur van de aortawand laat geen verschillen zien tussen ratten aan het einde van de zwangerschap en niet-zwangere ratten. We speculeren dat de stijging in de compliance en distensibiliteit van de aorta samen met de afname in totale perifere weerstand in de vroege zwangerschap ervoor dienen de toename in het slagvolume te buffer- 
en, om op die manier een stijging van de bloeddruk en arteriële wandspanning te voorkomen.

Het is beschreven dat de circulatie in de vroege zwangerschap, die gekenmerkt wordt door een hoge bloeddoorstroming en een lage weerstand, gepaard gaat met een toegenomen plasma renine activiteit. Dit suggereert dat de hemodynamiek in de vroege zwangerschap geassocieerd is met een relatieve vasculaire ondervulling. Echter, deze relatieve vasculaire ondervulling leidt in de rat niet tot een stijging van de hartfrequentie, hetgeen suggereert dat de reflexregulatie van de hartfrequentie veranderd is in de vroege zwangerschap.

IN HOOFDSTUK 4 wordt de regulatie van hartfrequentie en bloeddruk in de zwangerschap van de rat bestudeerd. Spectraalanalyse van spontaan voorkomende variaties in bloeddruk en hartfrequentie, een indicatie voor de activiteit van het autonoom zenuwstelsel, laat geen veranderingen zien in de vroege zwangerschap. De daling in afterload in de vroege zwangerschap in de rat lijkt dus noch te leiden tot een relatieve vasculaire ondervulling, noch tot een activering van het sympatisch zenuwstelsel.

HOOFDSTUK 5 beschrijft de veranderingen in de volumehomeostase in de vroege zwangerschap. Volume-expansie zal leiden tot een stijging van de preload, en zo tot een stijging van het slagvolume en het hartminuutvolume. Expansie van het plasmavolume in de vroege zwangerschap kan tot stand komen door vochtretentie en/of een verschuiving van extracellulaire vloeistof naar het vasculaire compartiment. De geleidelijke synchrone toename in het totale lichaamswater, extracellulaire vloeistof en plasmavolume in deze studie suggereert dat de toename in plasmavolume in de vroege zwangersehap het resultaat is van expansie van het extracellulaire volume door vloeistofretentie en niet door herverdeling van het extracellulaire volume.

In het tweede deel van dit proefschrift worden de factoren die verantwoordelijk kunnen zijn voor de initiële aanpassingen in de zwangerschap onderzocht.

HOOFDSTUK 6 beschrijft de cardiovasculaire aanpassingen in schijnzwangere ratten. De schijnzwangere en zwangere rat hebben vrijwel identieke hemodynamische aanpassingen tot dag 9. Dit suggereert dat, tenminste in de rat, de trofoblast niet essentieel is voor de initiële aanpassingen in de zwangerschap. De gelijkenis tussen de endocriene veranderingen in de vroege $z$ wangerschap van de rat en de schijnzwangere rat, ondersteunt de visie dat moederlijke hormonen 
betrokken zijn bij de eerste hemodynamische aanpassingen. Oestradiol-17\& is een potentiële kandidaat vanwege zijn welbekende vaatverwijdende effecten.

IN HOOPDSTUK 7 wordt de hypothese getest dat de vroegste toename in circulerende oestradiol-17B spiegels in de zwangerschap van de rat de initiële algehele vasorelaxatie van de zwangerschap induceert, hetgeen weerspiegeld zal worden in een hogere bloeddoorstroming van zowel de nier als het skeletweefsel. Onze bevinding dat oestradiol-17B vasodilatatie induceert in de vaten die het skeletweefsel en de huid voorzien, maar niet in de nier, suggereert dat oestradiol$17 \mathrm{~B}$ alleen niet direct verantwoordelijk is voor de initiële zwangerschapsgerelateerde vasorelaxatie.

De studies in dit proefschrift leiden tot de conclusie dat het hartminuutvolume toeneemt tussen dag 6 en dag 8 van de zwangerschap van de rat, ten gevolge van een stijging van het slagvolume. De stijging van het slagvolume is op haar beurt geïnduceerd door een daling van de afterload in de vroege zwangerschap, ten gevolge van een gecombineerde daling van de perifere weerstand en een stijging van de compliance van de aorta. De activering van volumeretentie zal leiden tot een stijging van het plasmavolume, waarschijnlijk door vloeistof- en zoutretentie. De toename van de cardiale contractiliteit kan het gevolg zijn van het hogere slagvolume dat geëjecteerd moet worden. Echter, een directe endocriene invloed op het hart door bijvoorbeeld oestradiol-17B kan niet uitgesloten worden. De trofoblast is niet essentieel voor de initiatie van de cardiovasculaire en renale aanpassingen aan de zwangerschap, omdat deze veranderingen ook bij schijnzwangere ratten worden waargenomen. Oestradiol-17ß alleen kan niet verantwoordelijk gehouden worden voor de gegeneraliseerde vasorelaxatie in de zwangerschap, maar kan een belangrijke rol spelen in het vrijkomen van potente vasodilatoren. 


\section{Publications}




\section{Scientific Abstracts}

B. Slangen, C. Weaver, C. Baylis. Renal effects of low dose Nitric Oxide (NO) inhibition in the rat. The American Society of Nephrology, 26th annual meeting, November 14-17, 1993, Boston.

Brigitte F.M. Slangen, Carla M. Verkeste and Louis L.H. Peeters. 17 B-estradiol (E2) has a dual effect on central hemodynamics in the awake ovariectomized rat. Proceedings of the 41st annual meeting of the SGI, Chicago, USA: 204.

Carla M. Verkeste, Brigitte F.M. Slangen and Louis L.H. Peeters. Ketanserin, in low (LD) and high dose (HD), compromizes uteroplacental blood flow (PBF) in the awake near-term rat. Proceedings of the 41 st annual meeting of the SGI, Chicago, USA: 387.

Brigitte F.M. Slangen, Iris C.M. Out, Carla M. Verkeste and Louis L.H. Peeters. The mechanism of early hemodynamic adaptation in rat pregnancy. J. Soc. Gynecol. Invest. vol. 2, No. 2: P159, 1995.

Brigitte F.M. Slangen, Iris C.M. Out, Carla M. Verkeste and Louis L.H. Peeters. Hemodynamic changes in awake early pregnant rats. Pflügers Arch.- Eur. J. Physiol. vol. 420, no 4: R117 (430), 1995.

C. Verkeste, B. Slangen, B. Janssen, L. Peeters. An indication of autoregulation of placental blood flow ?. Placenta 16: A.72, 1995.

C. Verkeste, M. Daemen, B. Slangen, H. van Straaten, G. Kohnen, L. Peeters. Pregnancy-induced structural alterations in the preplacental vasculature of the guinea pig. Placenta 16: A.72, 1995.

C. Verkeste, M. Daemen, B. Slangen, H. van Straaten, G. Kohnen, L.L.H. Peeters. Progressive structural changes also in the radial artery during guinea pig pregnancy. Am. J. Obstet. Gynecol., 174: 360, 1996. 
Brigitte F.M. Slangen, Iris C.M. Out, Ben J.A. Janssen, and Louis L.H. Peeters. Blood pressure and heart rate variability in pregnant rats. J. Soc. Gynecol. Invest. 1: $251 \mathrm{~A}, 1997$.

C.M. Verkeste, B.F.M. Slangen, M.L. Dubelaar, B.K. van Kreel, and L.L.H. Peeters. Gestational rise in plasma volume established by expansion of extracellular volume in the awake early pregnant rat. J. Soc. Gynecol. Invest. 1: 245A, 1997.

\section{Articles}

Brigitte F.M. Slangen, Iris C.M. Out, Carla M. Verkeste, and Louis L.H. Peeters. Hemodynamic changes in early pregnancy in chronically instrumented, conscious rats. Am. J. Physiol. 270: H1779-H1784, 1996.

C. Baylis, B. Slangen, S. Hussain, and C. Weaver. Relationship between basal NO release and cyclooxygenase products in the normal rat kidney. Am. J. Physiol. 271: R1327-R1334, 1996.

Brigitte F.M. Slangen, Iris C.M. Out, Carla M. Verkeste, Jos F.M. Smits and Louis L.H. Peeters. Hemodynamic changes in pseudopregnancy in chronically instrumented, conscious rats. Am. J. Physiol. 272: H695-H700, 1997.

Brigitte F.M. Slangen, Dorette S. van Ingen Schenau, Ad W. van Gorp, Jo G.R. De Mey and Louis L.H. Peeters. Aortic distensibility and compliance in awake pregnant rats. Am. J. Physiol., 272: H1260-H1265, 1997.

Carla M. Verkeste, Brigitte F.M. Slangen, Mat Daemen, Henny van Straten, Gaby Kohnen, Peter Kaufmann and Louis L.H. Peeters. The extent of trophoblast invasion in the preplacental vasculature of the guinea pig. Placenta, in press.

Brigitte F.M. Slangen, Iris C.M. Out, Ben J.A. Janssen and Louis L.H. Peeters. Blood pressure and heart rate variability in early pregnancy in the rat. Am. J. Physiol., in press. 


\section{Curriculum Vitae}

Brigitte Slangen werd op 11 mei 1968 in de Vroedvrouwenschool te Heerlen geboren. Van 1986 tot 1992 bezocht zij het Eykhagencollege te Schaesberg, waar zij cum laude slaagde yoor het Atheneum. Aansluitend startte zij met haar studie Geneeskunde aan de Rijksuniversiteit Limburg te Maastricht. In 1992 startte zij met de studies beschreven in dit proefschrift. Van oktober 1996 tot juli 1997 werkte zij als assistent geneeskundige bij de afdeling Gynaecologie en Obstetrie in het Maaslandziekenhuis te Sittard. Sinds 1 juli 1997 is zij in opleiding tot gynaecologe in het Academisch Ziekenhuis Maastricht (opleider Prof. Dr J. de Haan). 


\section{Nawoord}

Velen zijn, al dan niet gewild, betrokken geraakt bij dit onderzoek. Ik hoop dat ik mijn dankbaarheid in de afgelopen jaren al duidelijk heb laten blijken. Toch is het op zijn plaats een aantal mensen nogmaals te bedanken:

Allereerst Dr L.L.H. Peeters. Beste Louis, min of meer toevallig, vlak voor mijn afstuderen, kwam ik bij je terecht. Ik denk dat je enorme enthousiasme tijdens ons eerste gesprek invloed op mijn keuze heeft gehad. Door ook de gehele onderzoekstijd enthousiast en kritisch te blijven kijk ik tevreden terug op de afgelopen vier jaren, en blijf ik 'de wetenschap' een warm hart toedragen.

Prof. Dr J. de Haan. Beste professor, op de achtergrond keek u mee met mijn onderzoek, informeerde geregeld naar de voortgang, en hoe het met dit 'meisje' ging. Bedankt voor de steun.

Dr Ing. C.M. Verkeste, beste Carla, bedankt voor de geduldige wijze waarop je me de eerste stappen in het lab hebt leren zetten, voor de gezelligheid en voor het delen van alle frustaties inherent aan het onderzoek.

De vakgroep farmacologie onder leiding van Prof. Dr H.A.J. Struyker Boudier: Dr J.F.M. Smits, Jacques Debets, Caroline Eerdmans, Elsbeth Raes: zonder jullie ervaring en bereidheid deze met me te delen, waren een groot deel van deze studies niet gelukt.

Dr B.J.A. Janssen, Ben, je enthousiasme en ideeën over dit onderwerp waren stimulerend. Bedankt voor de zeer prettige samenwerking.

De vakgroep fysiologie: Dr J.G.R., Jo, de Mey, Ad van Gorp, Dorette van Ingen Schenau, Gregorio Fazzi, bedankt voor alle hulp bij de studie in hoofdstuk 3. 
Alle medewerkers van de proefdiervoorzieningen bedankt voor de betrokkenheid bij mijn bezigheden tijdens de vele dagen en weekenden in het kleine lab in de kelder van de universiteit! Bedankt voor de verzorging van de ratten, met name Harrie Zeegers en Hub Simons.

De medewerkers van de instrumentele dienst, onder andere Jan Geilen, Peter Laudy, Maurice Huinck, stonden altijd direct klaar voor het repareren of installeren van (meet)apparatuur. Bedankt!

Iris Out, (en Lambert), als student-assistent, 'keuzeblokstudent' en in je vrije tijd heb je veel bijgedragen aan mijn proefschrift. Bedankt voor je onvoorwaardelijke inzet!

Het 'PERIM': Han, Jolanda, Marc en Ralph: bedankt voor de gezelligheid en vriendschap.

Han, Nieuw-Zeeland is veel te ver weg. Ik vind het jammer dat juist jij, mijn kamer-en 'lotgenoot', niet bij mijn promotie bent. Ik ken nog steeds niemand die zoveel scheldwoorden voor het woord computer kan bedenken.

Mijn paranimfen Ralph en Marc (en Miriam en Miranda): bedankt dat jullie zo enthousiast deze taak op jullie hebben genomen.

De gynaecologen en verpleging in het Maasland Ziekenhuis in Sittard bedank ik voor de uitstekende begeleiding van mijn eerste stappen terug naar 'de kliniek'.

De gynaecologen, assistenten, secretaresses en verpleegkundigen van het AZM bedank ik yoor hun belangstelling in de afgelopen jaren, en de samenwerking in de nog komende jaren.

Yvette Thijs (en Kees van Loon) bedankt voor het corrigeren van mijn Nederlandse samenvatting. Jeroen Thijs en Jeroen van Wijck, bedankt voor de mooie vormgeving van dit boekje.

De belangrijkste personen in mijn leven doe ik tekort door ze hier in enkele regels te bedanken. Lieve Carlo, mam (en Mien), Patricia (en Roger) en oma: bedankt voor de liefdevolle steun en interesse. Wat zouden pap en opa trots zijn geweest! 
De publikatie van dit proefschrift werd mede mogelijk gemaakt door:

Ferring BV

GlaxoWellcome

Medical Dynamics

Novo Nordisk

Organon Nederland BV

Pfizer

Pie Medical

Skalar Medical BV

Wyeth-Lederle 